\title{
Cytotoxic Polyprenylated Xanthones from the Resin of Garcinia
}

\section{hanburyi}

Si-Jia Tao, ' Shu-Hong Guan, ' Wei Wang, Zhi-Qiang Lu, ' Guang-Tong Chen, Na Sha, ${ }^{\dagger}$ Qing-Xi Yue, ${ }^{\dagger}$ Xuan Liu, ${ }^{\dagger}$ and De-An Guo, ${ }^{* \dagger}$

Shanghai Research Center for Modernization of Traditional Chinese Medicine, Shanghai Institute of Materia Medica, Shanghai Institutes for Biological Sciences, Chinese Academy of Sciences, Guo Shoujing Road 199, Zhangjiang, Shanghai 201203, People's Republic of China, and National Center for Natural Products

Research, Research Institute of Pharmaceutical Sciences, Department of Pharmacognosy, School of Pharmacy, University of Mississippi, MS 38677, USA

* To whom correspondence should be addressed. Tel: 86-21-50271516. Fax: 86-21-50272789. E-mail: gda5958@163.com.

'Shanghai Institute of Materia Medica.

* University of Mississippi. 
List of Supporting Information

Figure S1. ${ }^{1} \mathrm{H}$ NMR spectrum (400 $\mathrm{MHz}, \mathrm{CDCl}_{3}$ ) of gambospiroene (1)

Figure S2. ${ }^{13} \mathrm{C}$ NMR spectrum $\left(100 \mathrm{MHz}, \mathrm{CDCl}_{3}\right)$ of gambospiroene (1)

Figure S3. HSQC spectrum (100 MHz, $\left.\mathrm{CDCl}_{3}\right)$ of gambospiroene (1)

Figure S4. HMBC spectrum (100 MHz, $\mathrm{CDCl}_{3}$ ) of gambospiroene (1)

Figure S5. ${ }^{1} \mathrm{H}-{ }^{1} \mathrm{H}$ COSY spectrum (600 MHz, $\mathrm{CDCl}_{3}$ ) of gambospiroene (1)

Figure S6. ROESY spectrum (600 MHz, $\mathrm{CDCl}_{3}$ ) of gambospiroene (1)

Figure S7. ${ }^{1} \mathrm{H}$ NMR spectrum (400 MHz, $\mathrm{CDCl}_{3}$ ) of methyl 8,8a-dihydromorellate (2)

Figure S8. ${ }^{13} \mathrm{C}$ NMR spectrum (100 MHz, $\left.\mathrm{CDCl}_{3}\right)$ of methyl 8,8a-dihydromorellate (2)

Figure S9. HSQC spectrum (100 MHz, $\mathrm{CDCl}_{3}$ ) of methyl 8,8a-dihydromorellate (2)

Figure S10. HMBC spectrum (100 MHz, $\mathrm{CDCl}_{3}$ ) of methyl 8,8a-dihydromorellate (2)

Figure S11. ROESY spectrum (600 MHz, $\mathrm{CDCl}_{3}$ ) of methyl 8,8a-dihydromorellate (2)

Figure S12. ${ }^{1} \mathrm{H}$ NMR spectrum (400 MHz, $\left.\mathrm{CDCl}_{3}\right)$ of 3-O-geranylforbesione (3)

Figure S13. ${ }^{13} \mathrm{C}$ NMR spectrum $\left(100 \mathrm{MHz}, \mathrm{CDCl}_{3}\right)$ of 3-O-geranylforbesione (3)

Figure S14. HSQC spectrum (100 MHz, $\mathrm{CDCl}_{3}$ ) of 3-O-geranylforbesione (3)

Figure S15. HMBC spectrum (100 MHz, $\mathrm{CDCl}_{3}$ ) of 3-O-geranylforbesione (3)

Figure S16. ${ }^{1} \mathrm{H}-{ }^{1} \mathrm{H}$ COSY spectrum (600 MHz, $\mathrm{CDCl}_{3}$ ) of 3-O-geranylforbesione (3)

Figure S17. ${ }^{1} \mathrm{H}$ NMR spectrum (400 MHz, $\mathrm{CDCl}_{3}$ ) of gambogefic acid (4)

Figure S18. ${ }^{13} \mathrm{C}$ NMR spectrum $\left(100 \mathrm{MHz}, \mathrm{CDCl}_{3}\right)$ of gambogefic acid (4)

Figure S19. HSQC spectrum (100 MHz, $\mathrm{CDCl}_{3}$ ) of gambogefic acid (4)

Figure S20. HMBC spectrum (100 MHz, $\mathrm{CDCl}_{3}$ ) of gambogefic acid (4)

Figure S21. ${ }^{1} \mathrm{H}-{ }^{1} \mathrm{H}$ COSY spectrum (600 MHz, $\mathrm{CDCl}_{3}$ ) of gambogefic acid (4) 
Figure S22. ${ }^{1} \mathrm{H}$ NMR spectrum (400 MHz, $\left.\mathrm{CDCl}_{3}\right)$ of 7-methoxygambogellic acid (5)

Figure S23. ${ }^{13} \mathrm{C}$ NMR spectrum (100 MHz, $\mathrm{CDCl}_{3}$ ) of 7-methoxygambogellic acid (5)

Figure S24. HSQC spectrum (100 MHz, $\mathrm{CDCl}_{3}$ ) of 7-methoxygambogellic acid (5)

Figure S25. HMBC spectrum (100 MHz, $\mathrm{CDCl}_{3}$ ) of 7-methoxygambogellic acid (5)

Figure S26. ${ }^{1} \mathrm{H}-{ }^{1} \mathrm{H}$ COSY spectrum (600 MHz, $\mathrm{CDCl}_{3}$ ) of 7-methoxygambogellic acid (5)

Figure S27. ROESY spectrum (600 MHz, $\mathrm{CDCl}_{3}$ ) of 7-methoxygambogellic acid (5)

Figure S28. ${ }^{1} \mathrm{H}$ NMR spectrum (400 MHz, $\mathrm{CDCl}_{3}$ ) of 7-methoxygambogic acid (6)

Figure S29. ${ }^{13} \mathrm{C}$ NMR spectrum $\left(100 \mathrm{MHz}, \mathrm{CDCl}_{3}\right)$ of 7-methoxygambogic acid (6)

Figure S30. HSQC spectrum (100 MHz, $\mathrm{CDCl}_{3}$ ) of 7-methoxygambogic acid (6)

Figure S31. HMBC spectrum (100 MHz, $\mathrm{CDCl}_{3}$ ) of 7-methoxygambogic acid (6)

Figure S32. ${ }^{1} \mathrm{H}$ NMR spectrum (400 MHz, $\mathrm{CDCl}_{3}$ ) of 7-methoxyepigambogic acid (7)

Figure S33. ${ }^{13} \mathrm{C}$ NMR spectrum (100 MHz, $\mathrm{CDCl}_{3}$ ) of 7-methoxyepigambogic acid (7)

Figure S34. HMBC spectrum (100 MHz, $\left.\mathrm{CDCl}_{3}\right)$ of 7-methoxyepigambogic acid (7)

Figure S35. ${ }^{1} \mathrm{H}$ NMR spectrum (400 MHz, $\mathrm{CDCl}_{3}$ ) of 8,8a-dihydro-8-hydroxymorellic acid (8)

Figure S36. ${ }^{13} \mathrm{C}$ NMR spectrum $\left(100 \mathrm{MHz}, \mathrm{CDCl}_{3}\right)$ of 8,8a-dihydro-8-hydroxymorellic acid (8)

Figure S37. HSQC spectrum (100 MHz, $\mathrm{CDCl}_{3}$ ) of 8,8a-dihydro-8-hydroxymorellic acid (8)

Figure S38. HMBC spectrum (100 MHz, $\mathrm{CDCl}_{3}$ ) of 8,8a-dihydro-8-hydroxymorellic acid (8)

Figure S39. ROESY spectrum (600 MHz, $\mathrm{CDCl}_{3}$ ) of 8,8a-dihydro-8-hydroxymorellic acid (8)

Figure S40. ${ }^{1} \mathrm{H}$ NMR spectrum (400 $\mathrm{MHz}, \mathrm{CDCl}_{3}$ ) of 8,8a-dihydro-8-hydroxygambogenic acid

Figure S41. ${ }^{13} \mathrm{C}$ NMR spectrum $\left(100 \mathrm{MHz}, \mathrm{CDCl}_{3}\right)$ of 8,8a-dihydro-8-hydroxygambogenic acid 
Figure S42. HSQC spectrum (100 MHz, $\mathrm{CDCl}_{3}$ ) of 8,8a-dihydro-8-hydroxygambogenic acid (9)

Figure S43. HMBC spectrum (100 MHz, $\mathrm{CDCl}_{3}$ ) of 8,8a-dihydro-8-hydroxygambogenic acid (9)

Figure S44. ROESY spectrum (600 MHz, $\mathrm{CDCl}_{3}$ ) of 8,8a-dihydro-8-hydroxygambogenic acid (9)

Figure S45. ${ }^{1} \mathrm{H}$ NMR spectrum (400 MHz, $\mathrm{CDCl}_{3}$ ) of oxygambogic acid (10)

Figure S46. ${ }^{13} \mathrm{C}$ NMR spectrum $\left(100 \mathrm{MHz}, \mathrm{CDCl}_{3}\right)$ of oxygambogic acid (10)

Figure S47. HSQC spectrum (100 MHz, $\mathrm{CDCl}_{3}$ ) of oxygambogic acid (10)

Figure S48. HMBC spectrum (100 MHz, $\mathrm{CDCl}_{3}$ ) of oxygambogic acid (10)

Figure S49. ROESY spectrum (600 MHz, $\mathrm{CDCl}_{3}$ ) of oxygambogic acid (10)

Figure S50. ${ }^{1} \mathrm{H}$ NMR spectrum (400 MHz, $\mathrm{CDCl}_{3}$ ) of gambogenific acid (11)

Figure S51. ${ }^{13} \mathrm{C}$ NMR spectrum $\left(100 \mathrm{MHz}, \mathrm{CDCl}_{3}\right)$ of gambogenific acid (11)

Figure S52. HSQC spectrum (100 MHz, $\mathrm{CDCl}_{3}$ ) of gambogenific acid (11)

Figure S53. HMBC spectrum (100 MHz, $\left.\mathrm{CDCl}_{3}\right)$ of gambogenific acid (11)

Figure S54. ${ }^{1} \mathrm{H}$ NMR spectrum $\left(300 \mathrm{MHz}, \mathrm{CDCl}_{3}\right.$ ) of 7-methoxyisomorellinol (12)

Figure S55. ${ }^{13} \mathrm{C}$ NMR spectrum (100 MHz, $\mathrm{CDCl}_{3}$ ) of 7-methoxyisomorellinol (12)

Figure S56. HSQC spectrum (100 MHz, $\mathrm{CDCl}_{3}$ ) of 7-methoxyisomorellinol (12)

Figure S57. ${ }^{1} \mathrm{H}$ NMR spectrum (400 MHz, $\mathrm{CDCl}_{3}$ ) of 8,8a-dihydro-8-hydroxygambogic acid (13)

Figure S58. ${ }^{13} \mathrm{C}$ NMR spectrum (100 MHz, $\left.\mathrm{CDCl}_{3}\right)$ of 8,8a-dihydro-8-hydroxygambogic acid (13)

Figure S59. ${ }^{1} \mathrm{H}$ NMR spectrum (400 $\mathrm{MHz}, \mathrm{CDCl}_{3}$ ) of 8,8a-dihydro-8-hydroxygambogic acid isomer (14)

Figure S60. ${ }^{13} \mathrm{C}$ NMR spectrum $\left(100 \mathrm{MHz}, \mathrm{CDCl}_{3}\right)$ of 8,8a-dihydro-8-hydroxygambogic acid isomer (14) 
Figure S1. ${ }^{1} \mathrm{H}$ NMR spectrum (400 MHz, $\mathrm{CDCl}_{3}$ ) of gambospiroene (1)

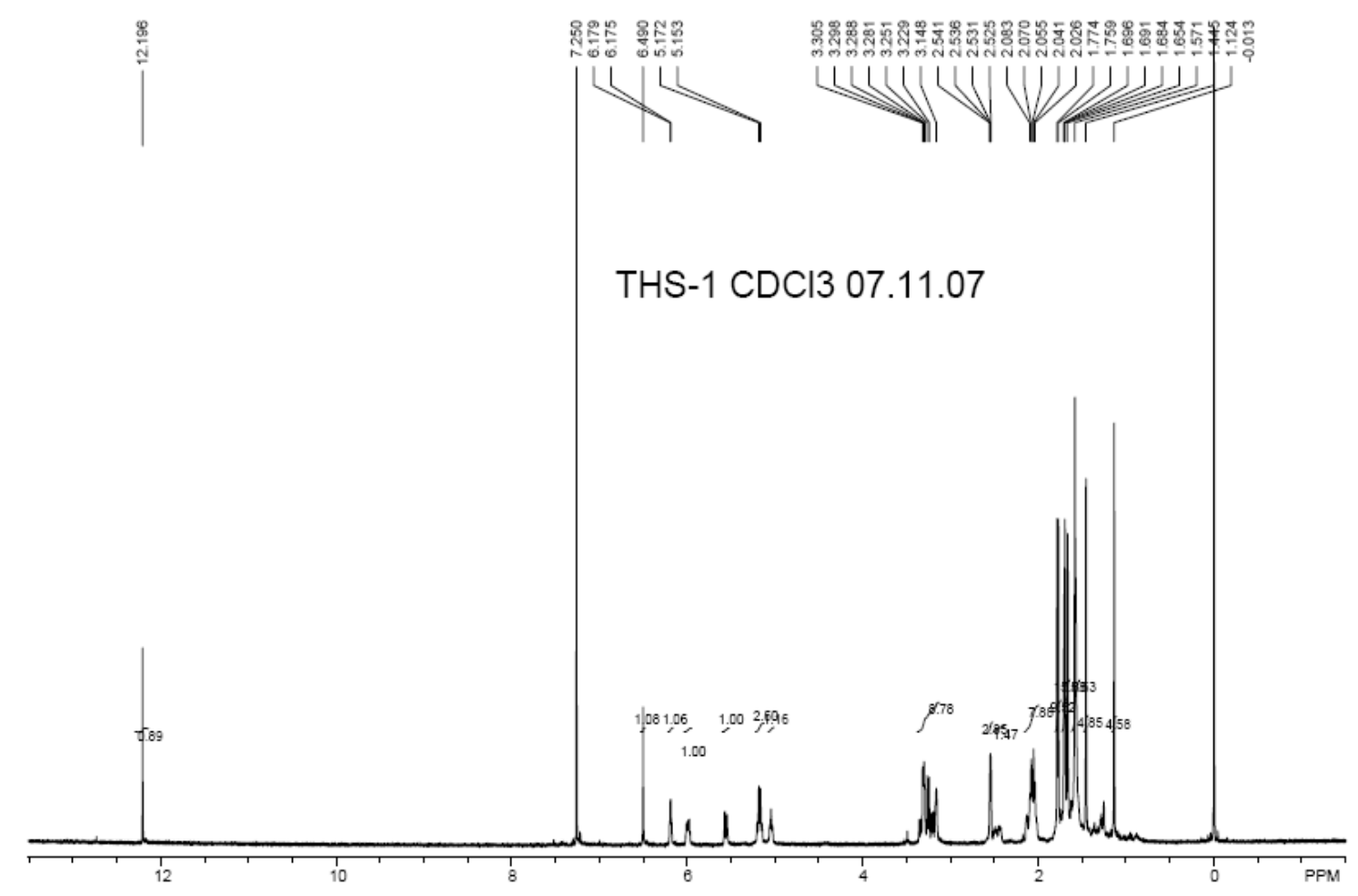

Figure S2. ${ }^{13} \mathrm{C}$ NMR spectrum $\left(100 \mathrm{MHz}, \mathrm{CDCl}_{3}\right)$ of gambospiroene (1)

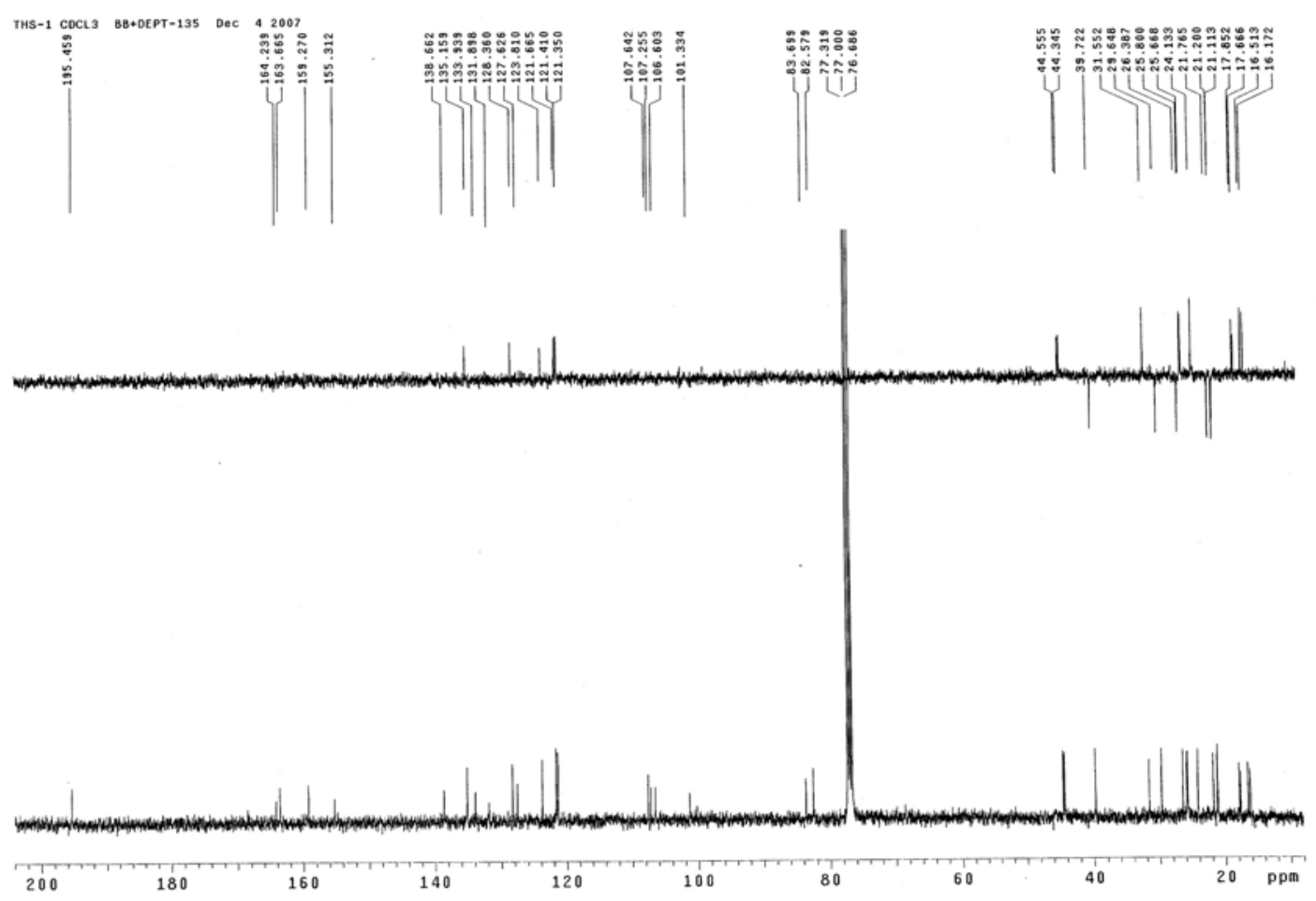


Figure S3. HSQC spectrum (100 MHz, $\left.\mathrm{CDCl}_{3}\right)$ of gambospiroene (1)

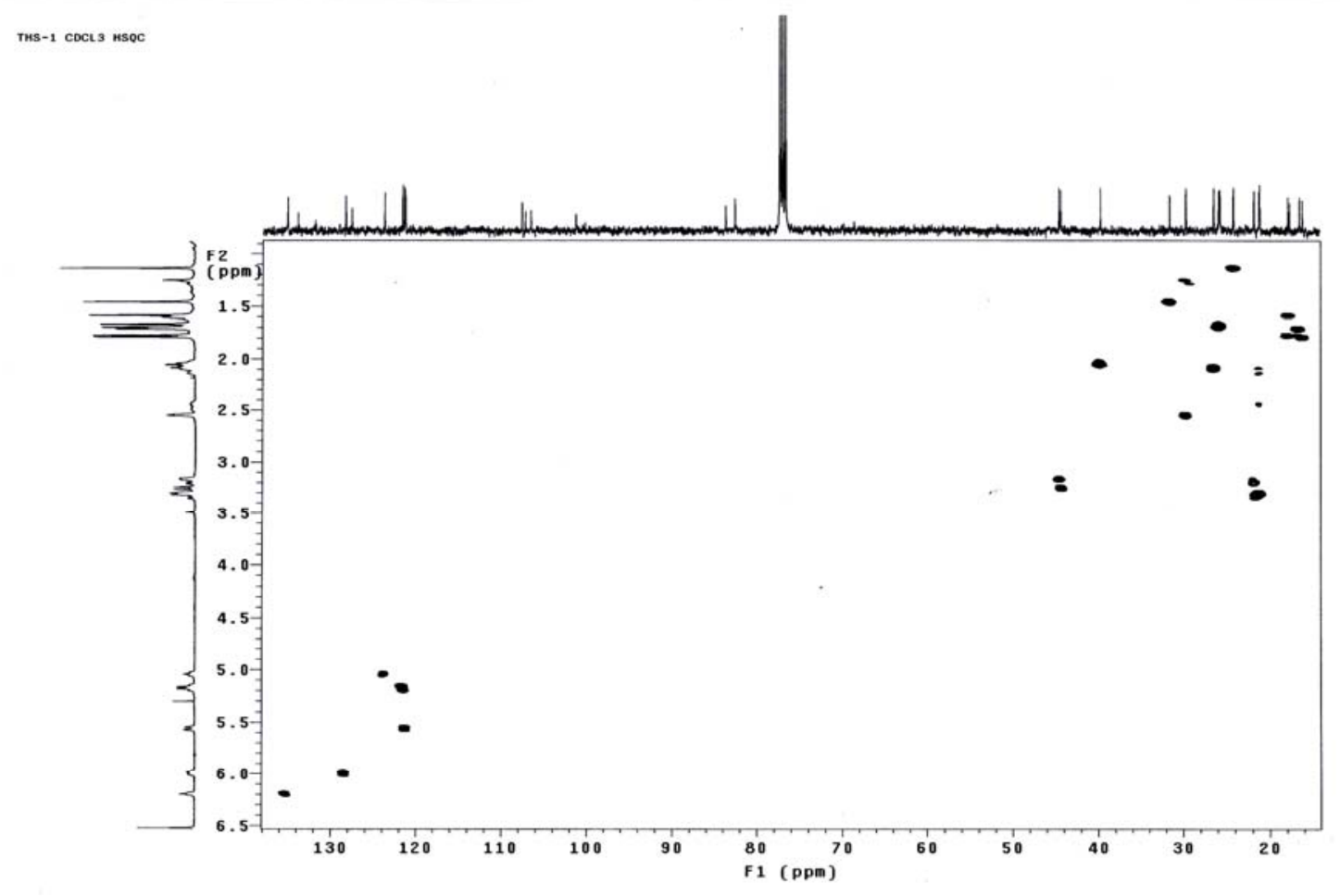

Figure S4. HMBC spectrum (100 MHz, $\left.\mathrm{CDCl}_{3}\right)$ of gambospiroene (1)

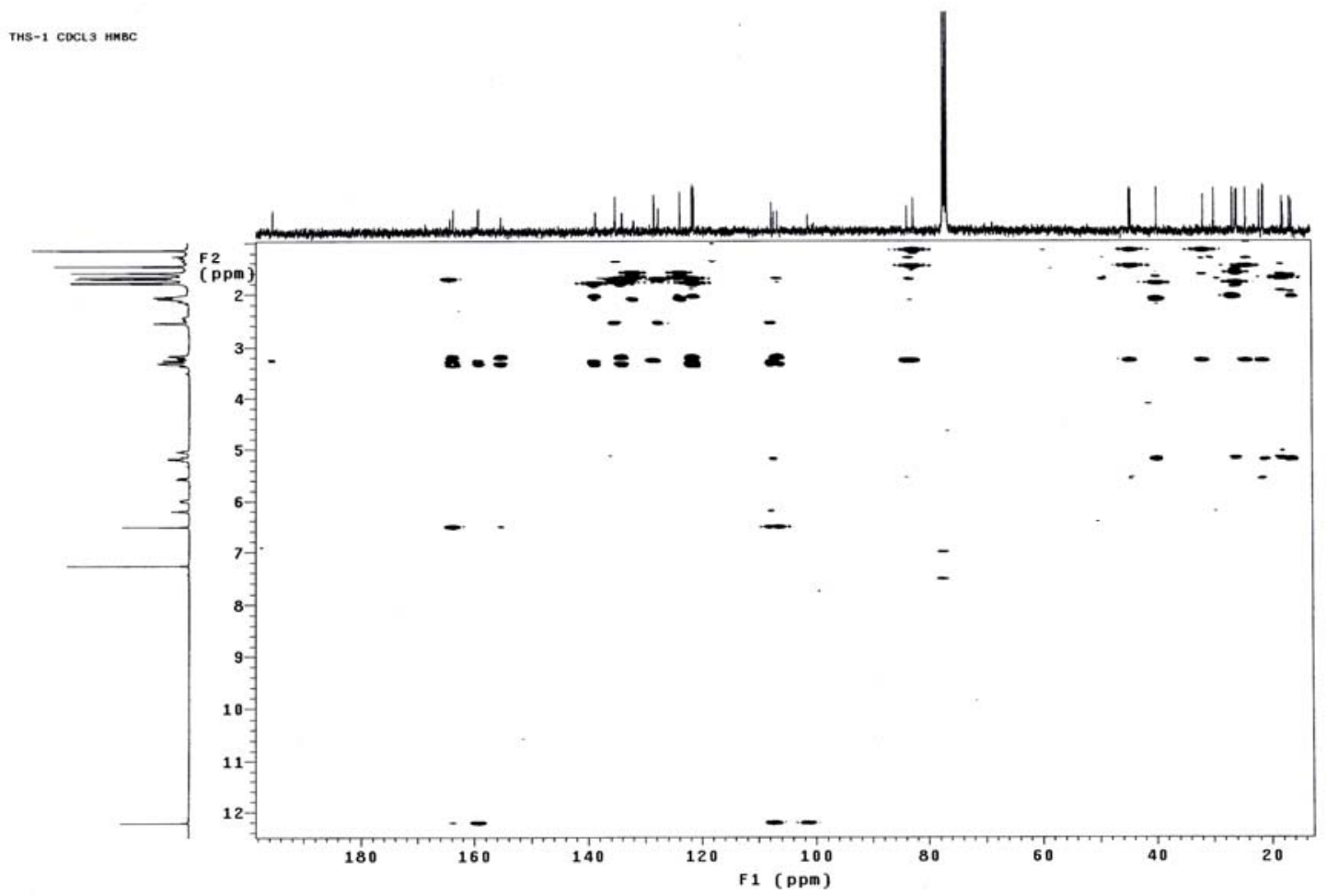


Figure S5. ${ }^{1} \mathrm{H}-{ }^{1} \mathrm{H}$ COSY spectrum (600 MHz, $\mathrm{CDCl}_{3}$ ) of gambospiroene (1)

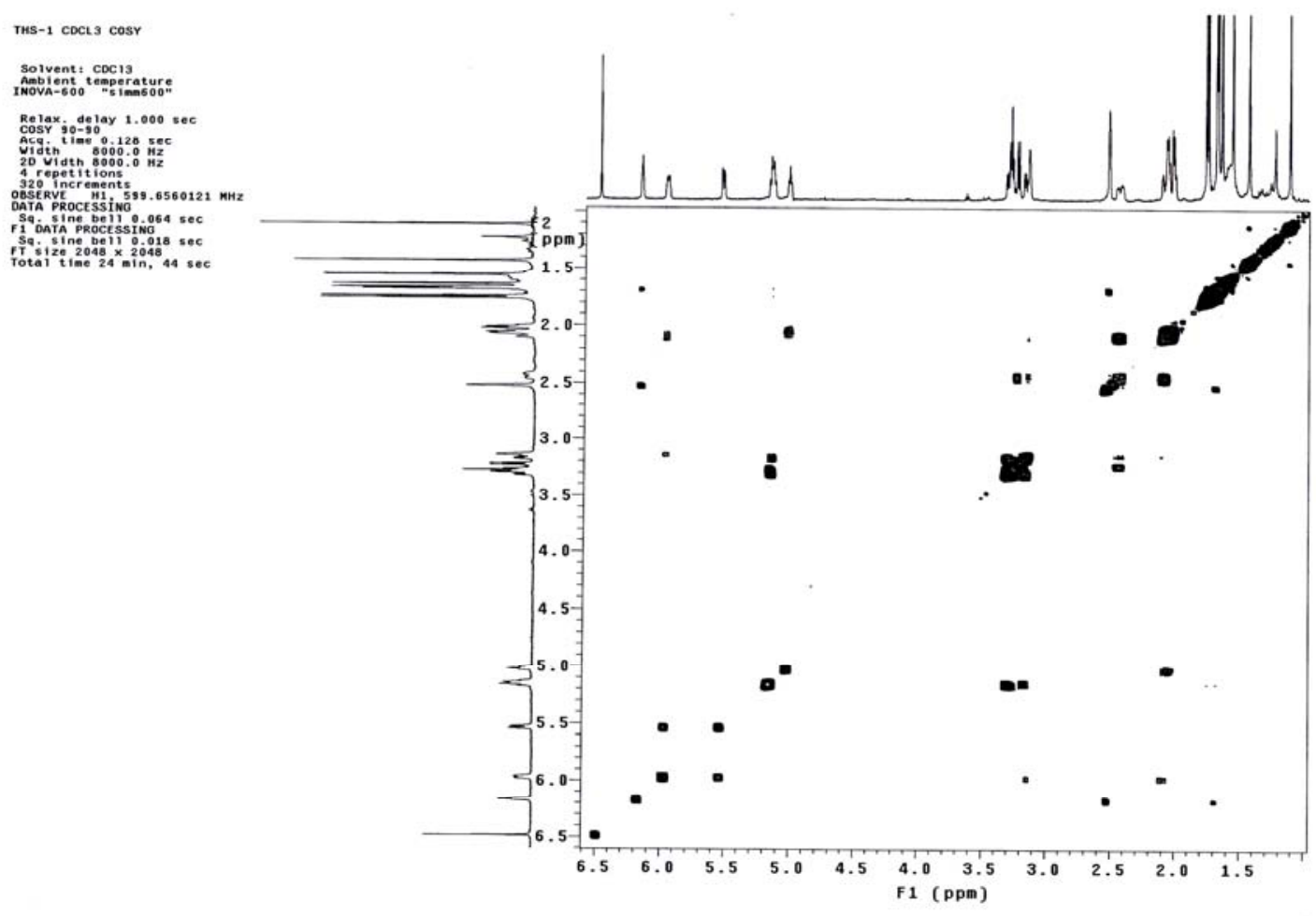

Figure S6. ROESY spectrum (600 MHz, $\left.\mathrm{CDCl}_{3}\right)$ of gambospiroene (1)

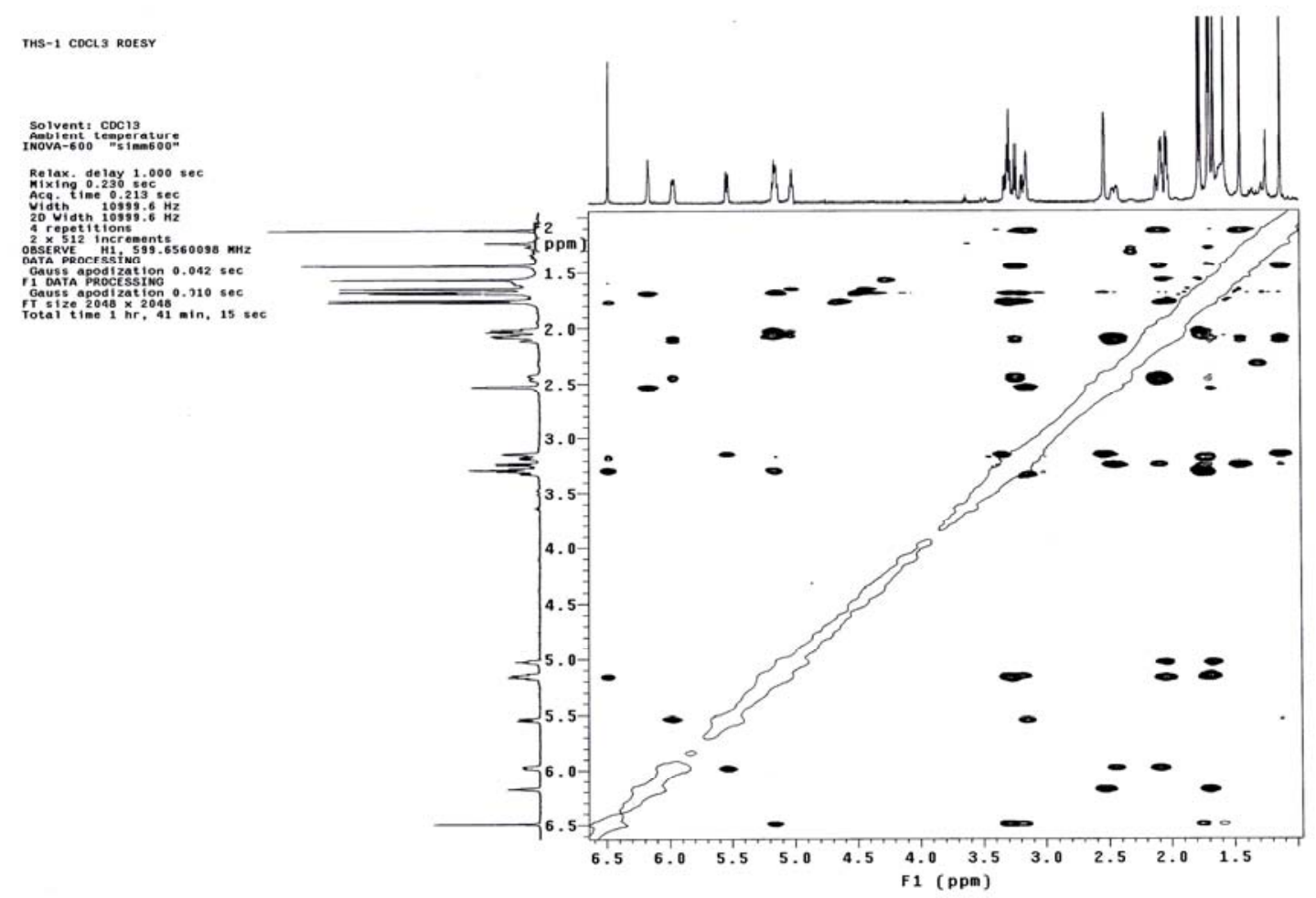


Figure S7. ${ }^{1} \mathrm{H}$ NMR spectrum (400 MHz, $\mathrm{CDCl}_{3}$ ) of methyl 8,8a-dihydromorellate (2)

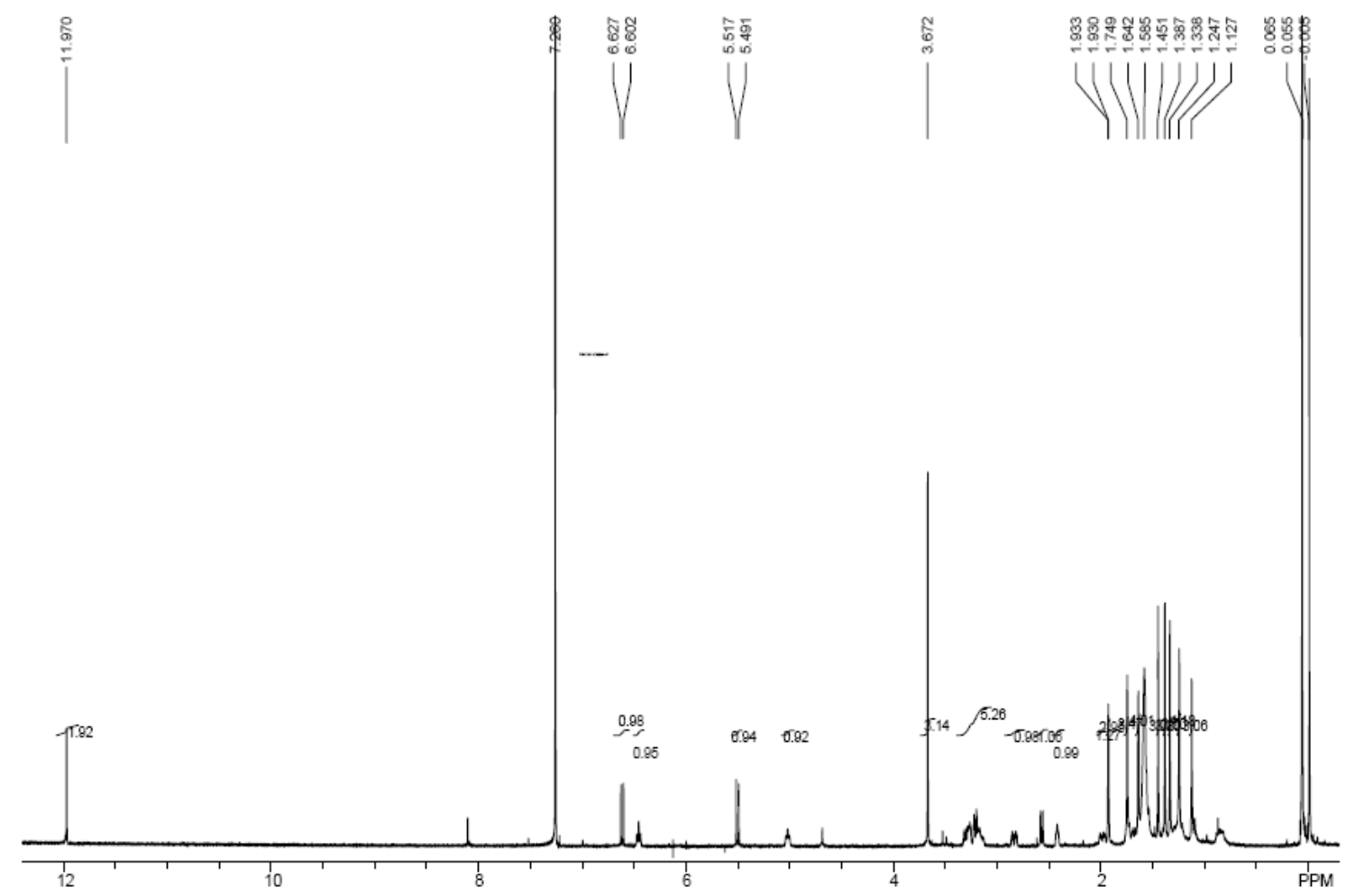

Figure S8. ${ }^{13} \mathrm{C}$ NMR spectrum (100 MHz, $\mathrm{CDCl}_{3}$ ) of methyl 8,8a-dihydromorellate (2)
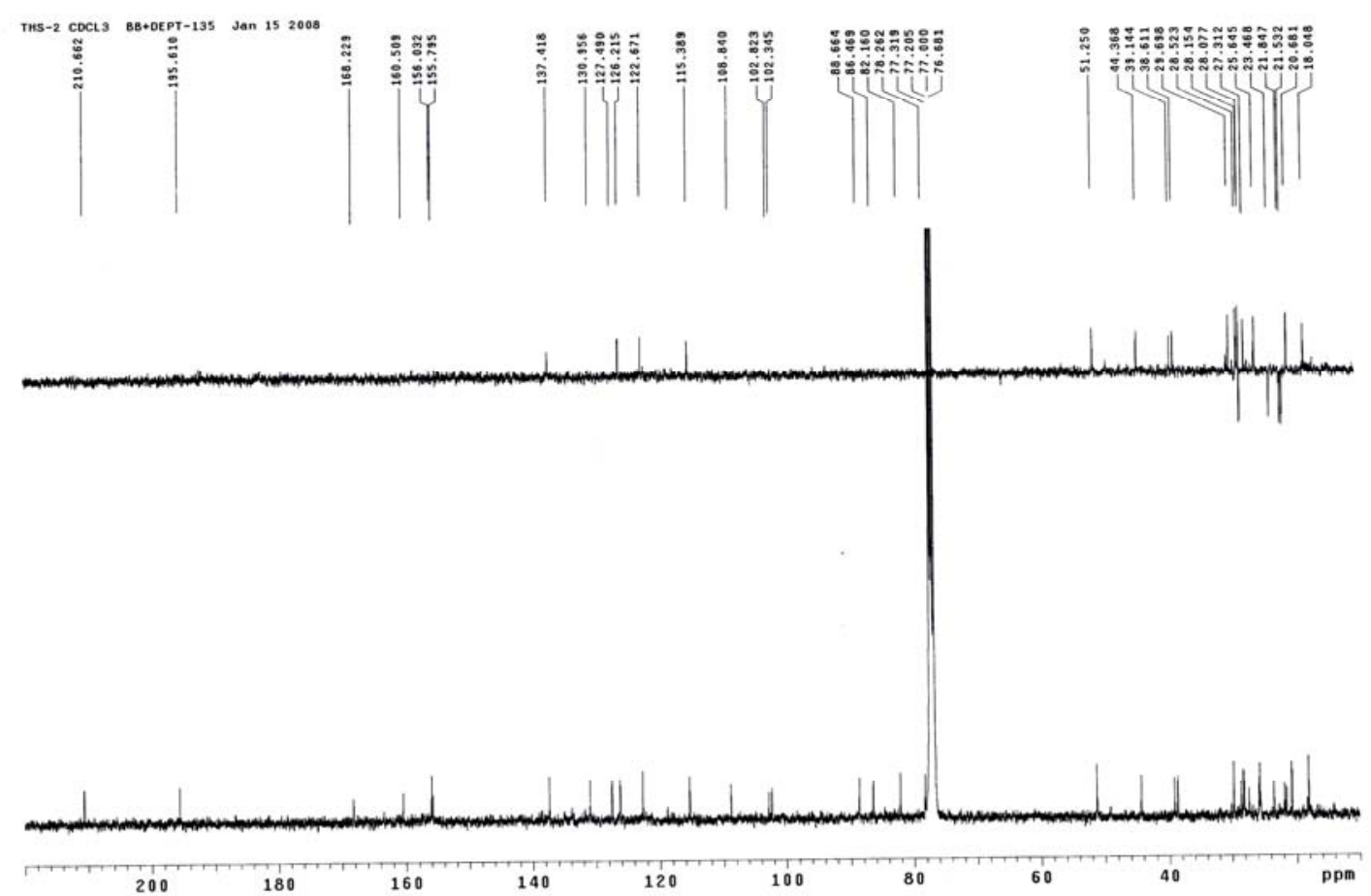
Figure S9. HSQC spectrum (100 MHz, $\mathrm{CDCl}_{3}$ ) of methyl 8,8a-dihydromorellate (2)

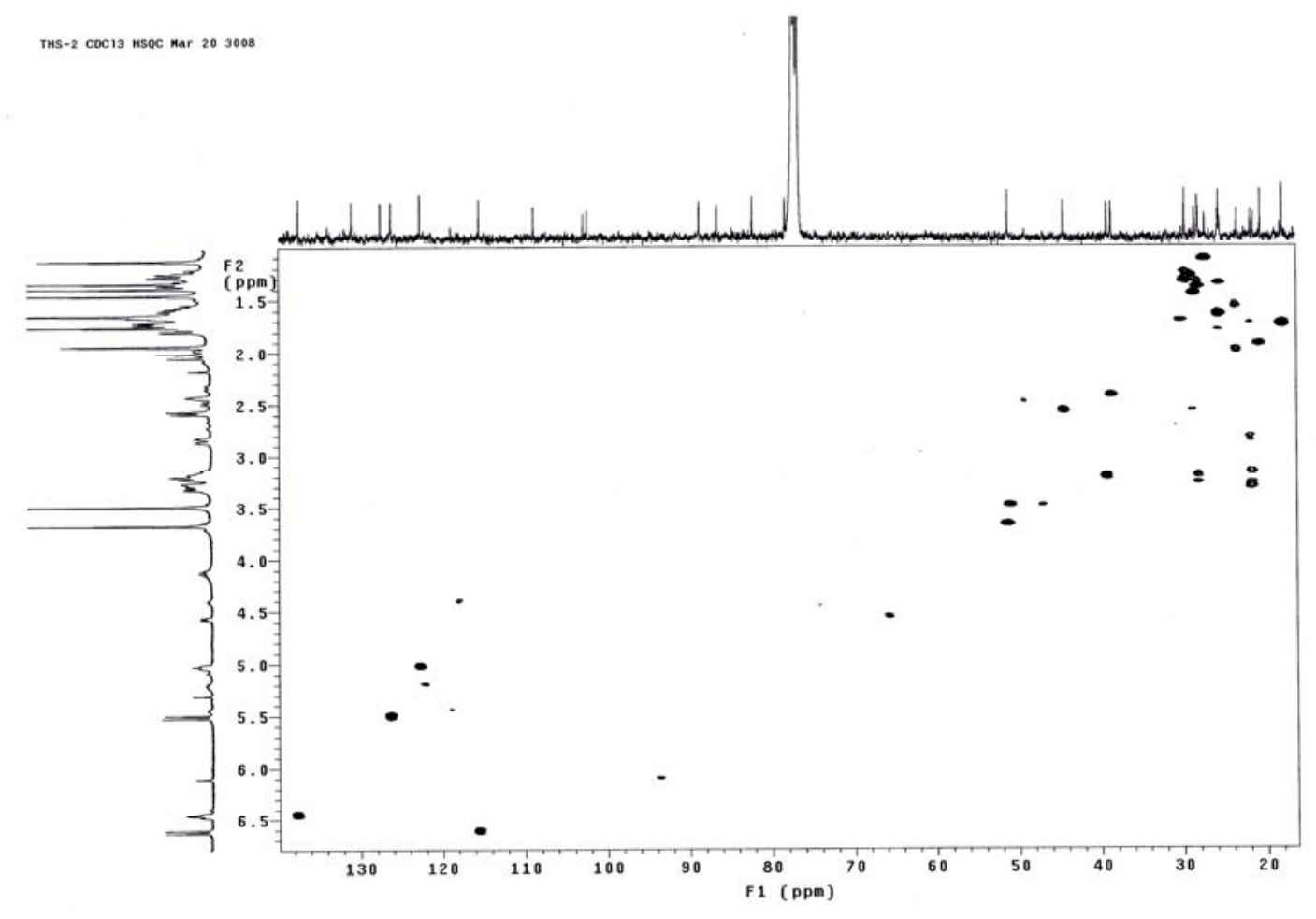

Figure S10. $\mathrm{HMBC}$ spectrum (100 MHz, $\mathrm{CDCl}_{3}$ ) of methyl 8,8a-dihydromorellate (2)

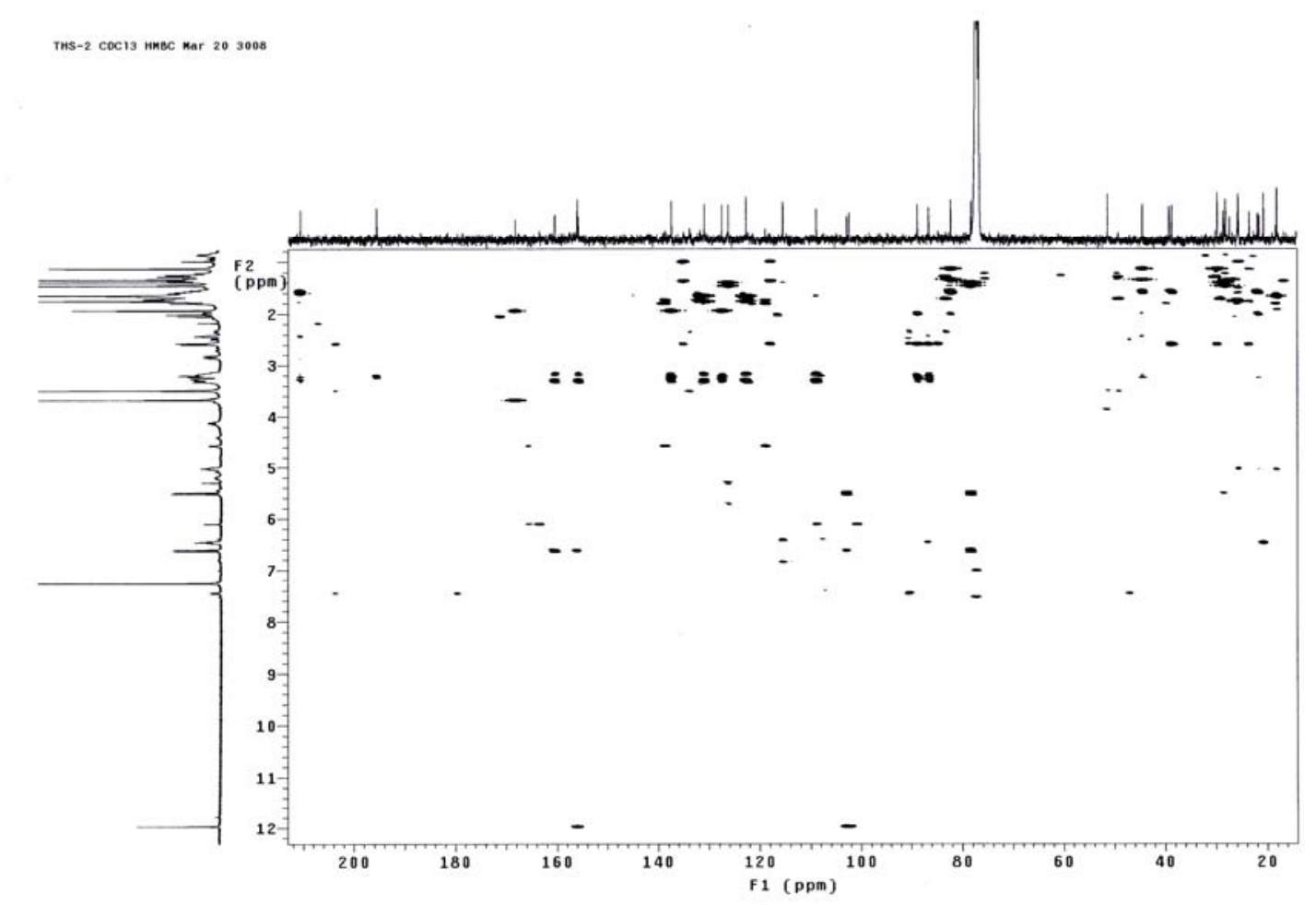


Figure S11. ROESY spectrum (600 MHz, $\mathrm{CDCl}_{3}$ ) of methyl 8,8a-dihydromorellate (2)

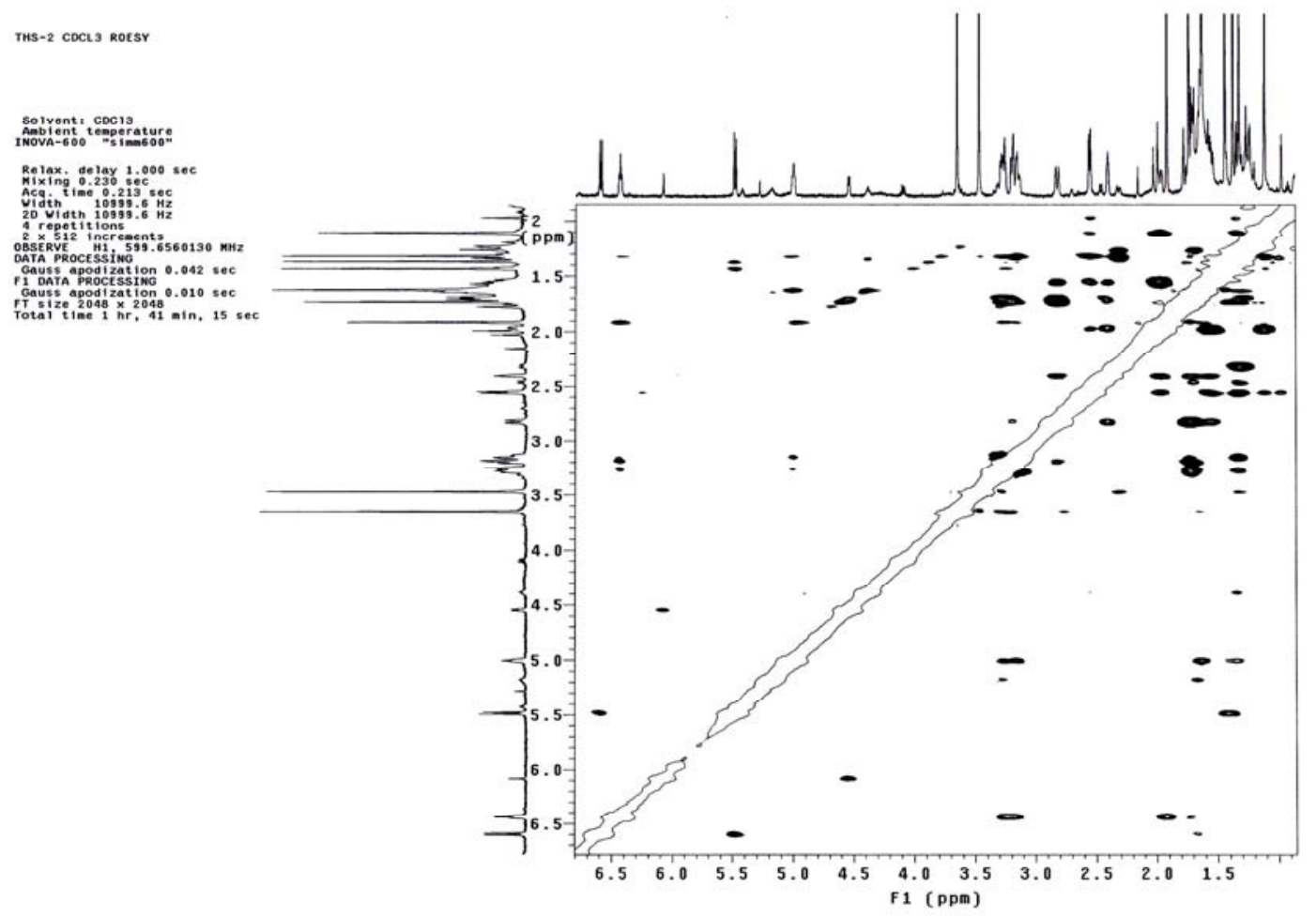

Figure S12. ${ }^{1} \mathrm{H}$ NMR spectrum (400 MHz, $\mathrm{CDCl}_{3}$ ) of 3-O-geranylforbesione (3)
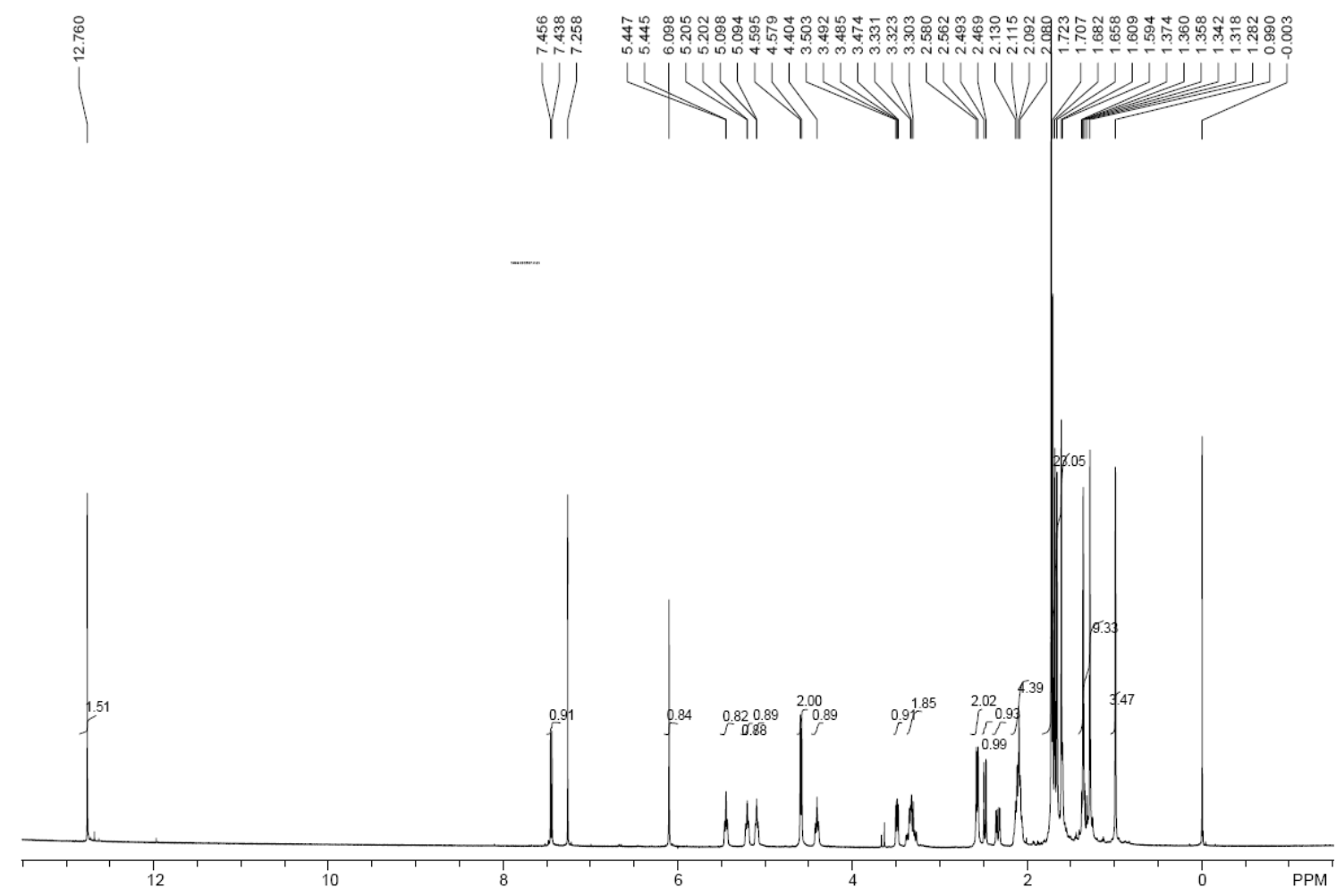
Figure S13. ${ }^{13} \mathrm{C}$ NMR spectrum $\left(100 \mathrm{MHz}, \mathrm{CDCl}_{3}\right)$ of 3-O-geranylforbesione (3)

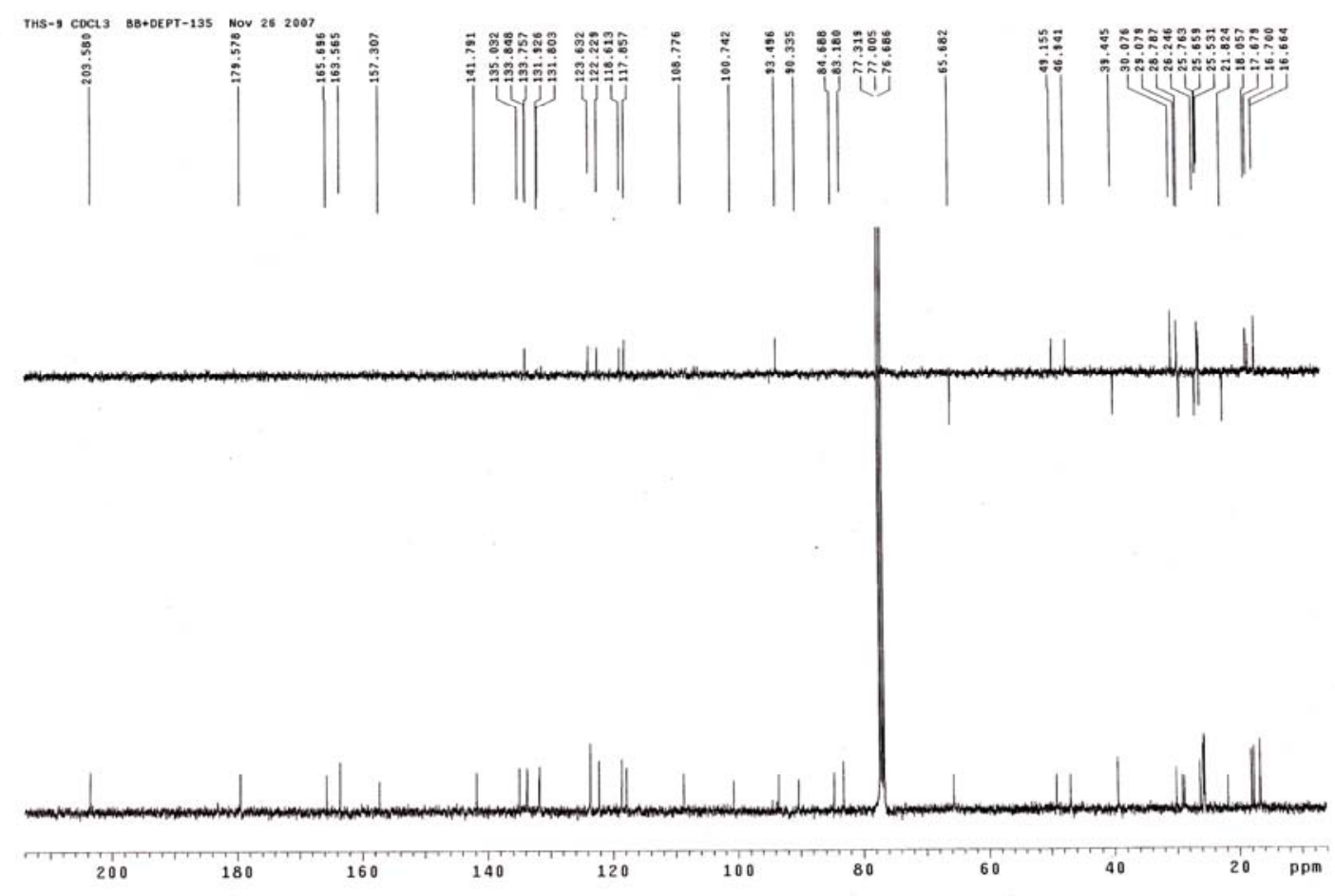

Figure S14. HSQC spectrum (100 MHz, $\mathrm{CDCl}_{3}$ ) of 3-O-geranylforbesione (3)

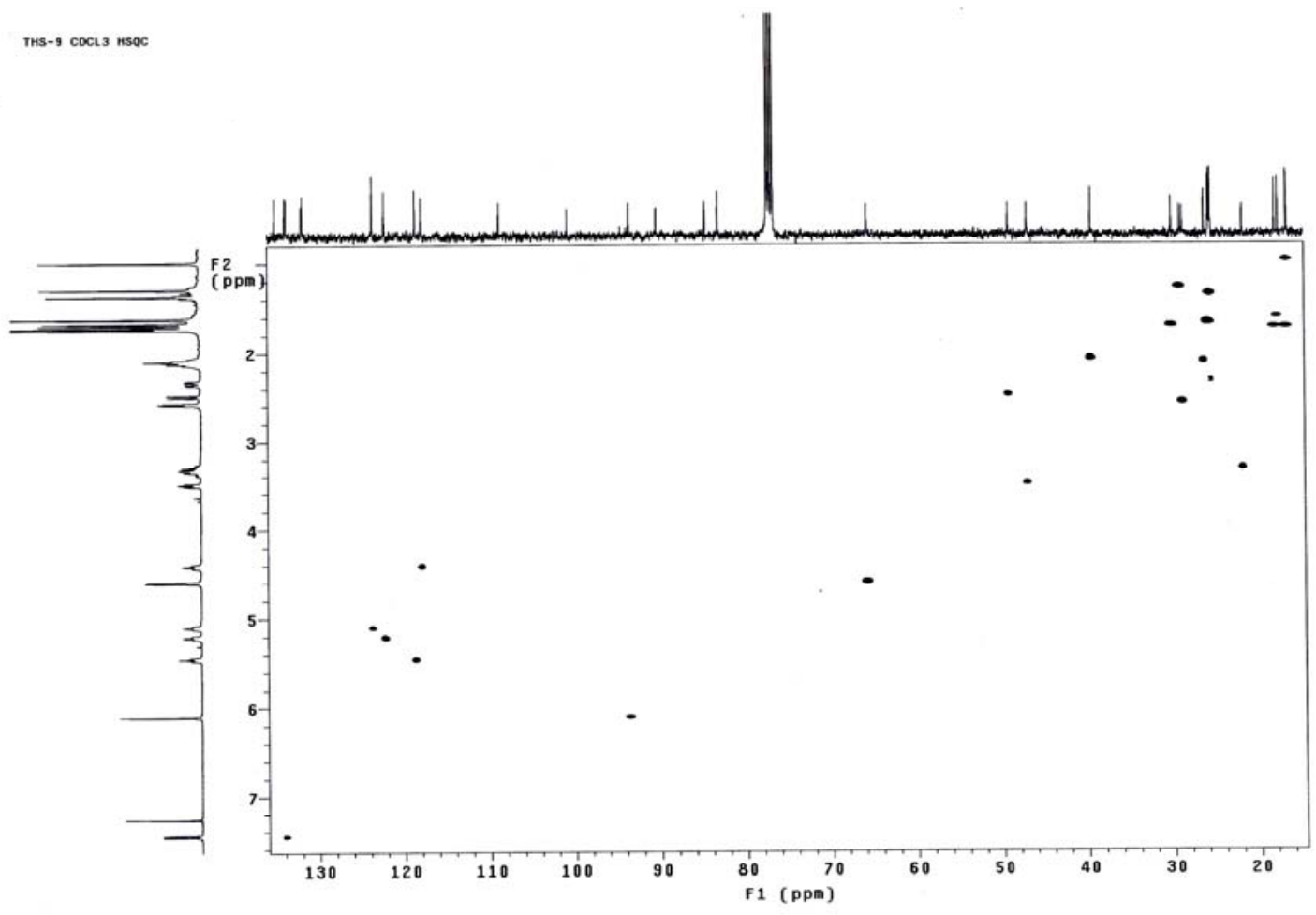


Figure S15. HMBC spectrum (100 MHz, $\mathrm{CDCl}_{3}$ ) of 3-O-geranylforbesione (3)

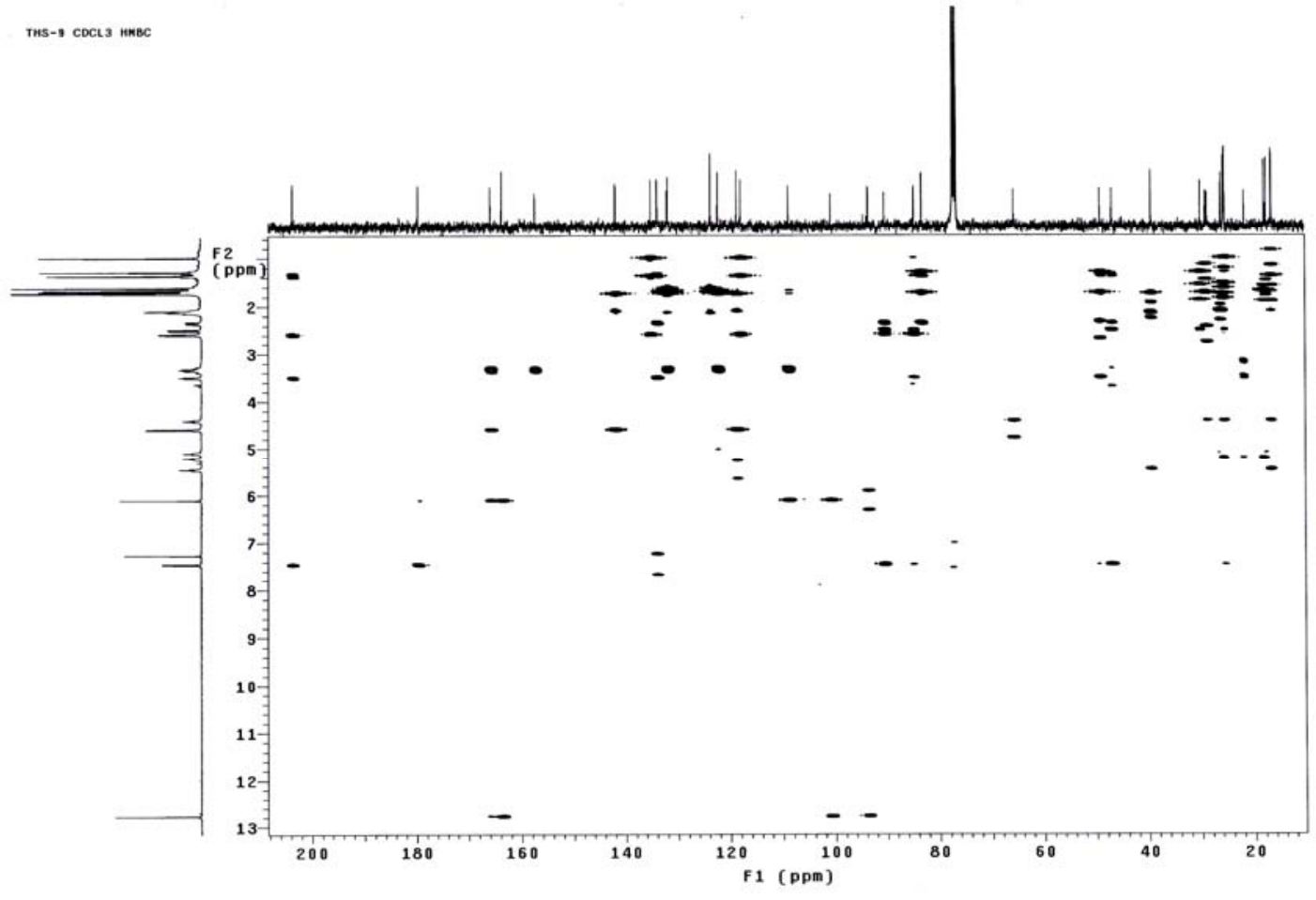

Figure S16. ${ }^{1} \mathrm{H}-{ }^{1} \mathrm{H}$ COSY spectrum $\left(600 \mathrm{MHz}, \mathrm{CDCl}_{3}\right)$ of 3-O-geranylforbesione (3)

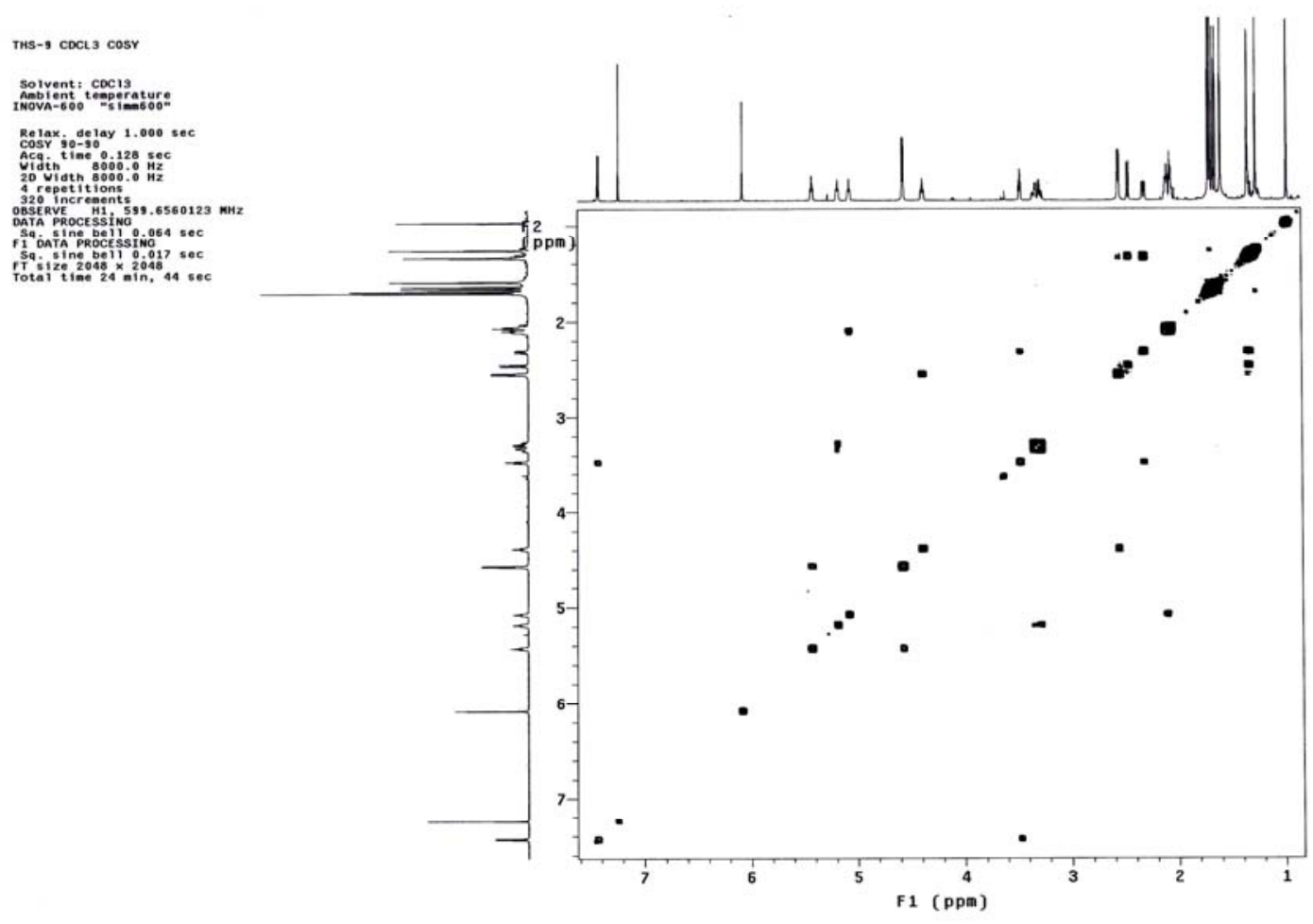


Figure S17. ${ }^{1} \mathrm{H}$ NMR spectrum (400 MHz, $\mathrm{CDCl}_{3}$ ) of gambogefic acid (4)

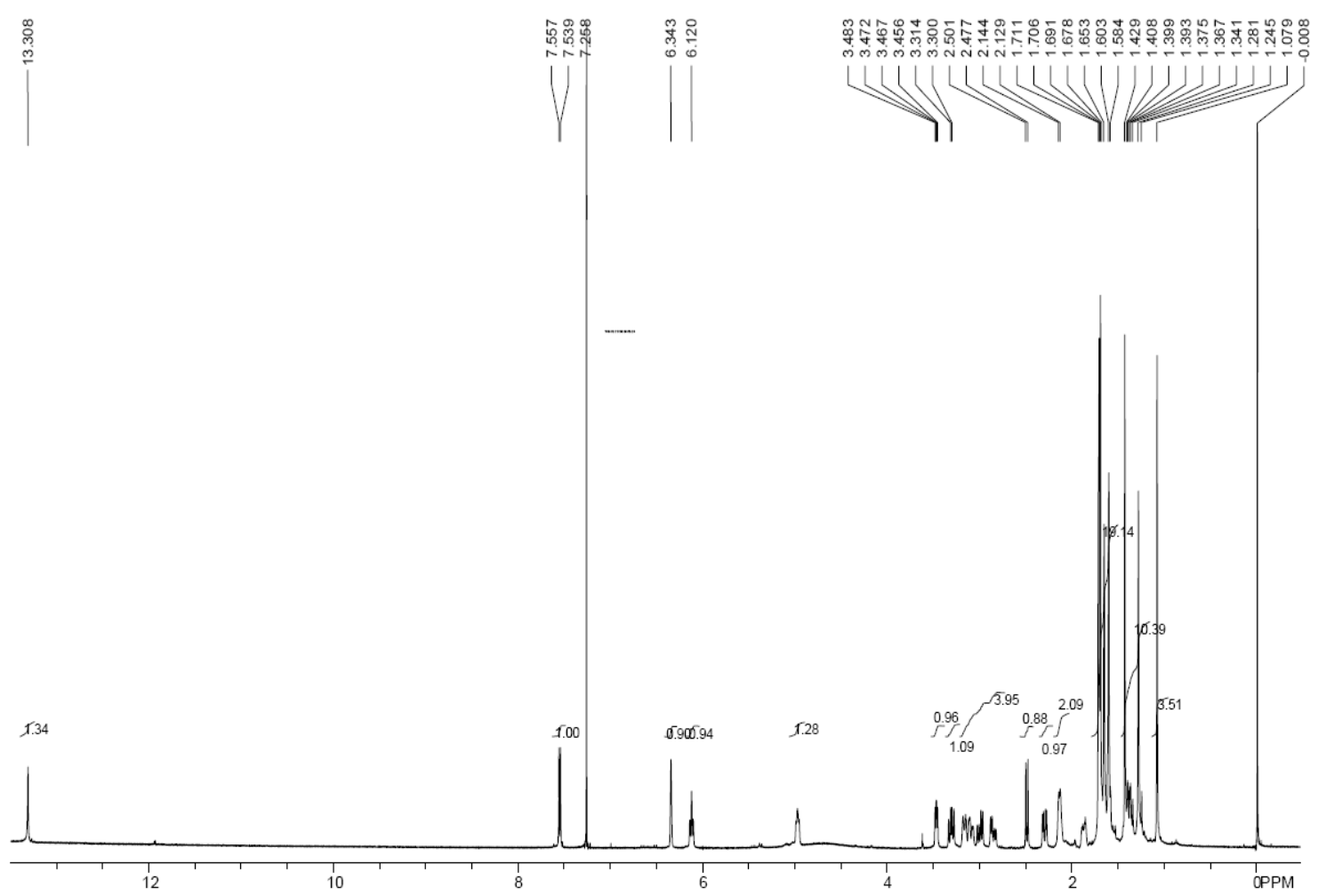

Figure S18. ${ }^{13} \mathrm{C}$ NMR spectrum $\left(100 \mathrm{MHz}, \mathrm{CDCl}_{3}\right)$ of gambogefic acid (4)

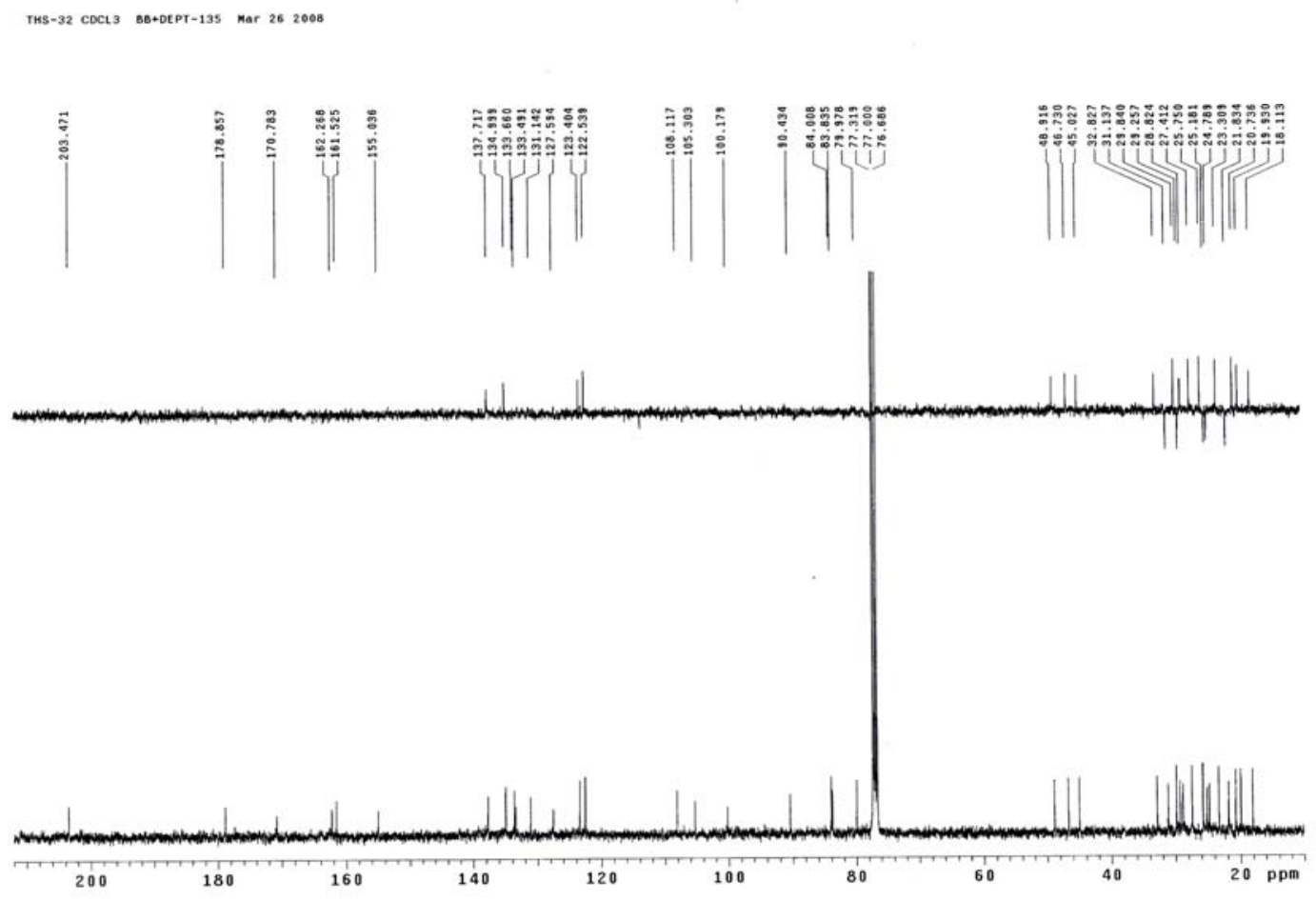


Figure S19. HSQC spectrum (100 MHz, $\mathrm{CDCl}_{3}$ ) of gambogefic acid (4)

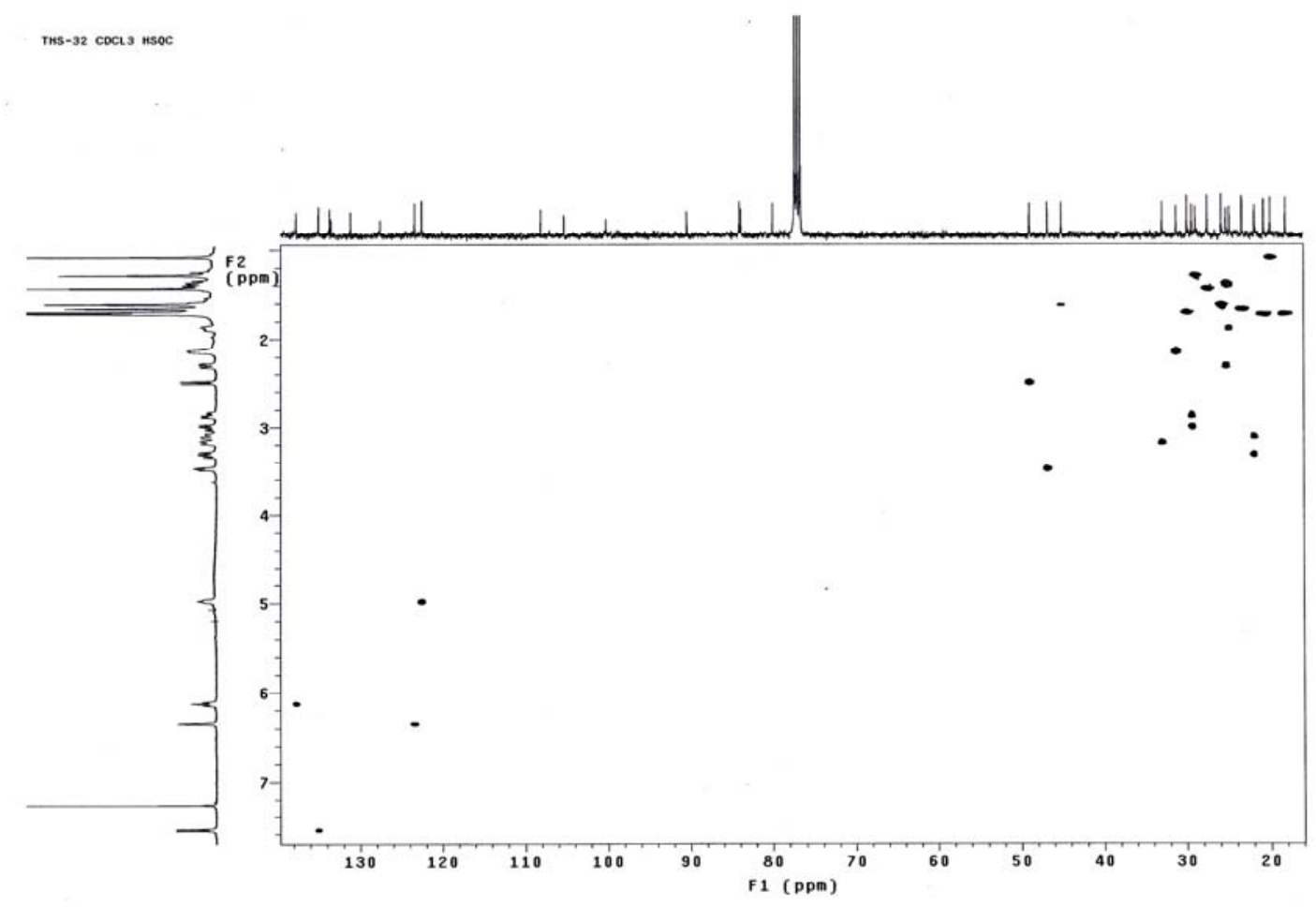

Figure S20. HMBC spectrum (100 MHz, $\mathrm{CDCl}_{3}$ ) of gambogefic acid (4)

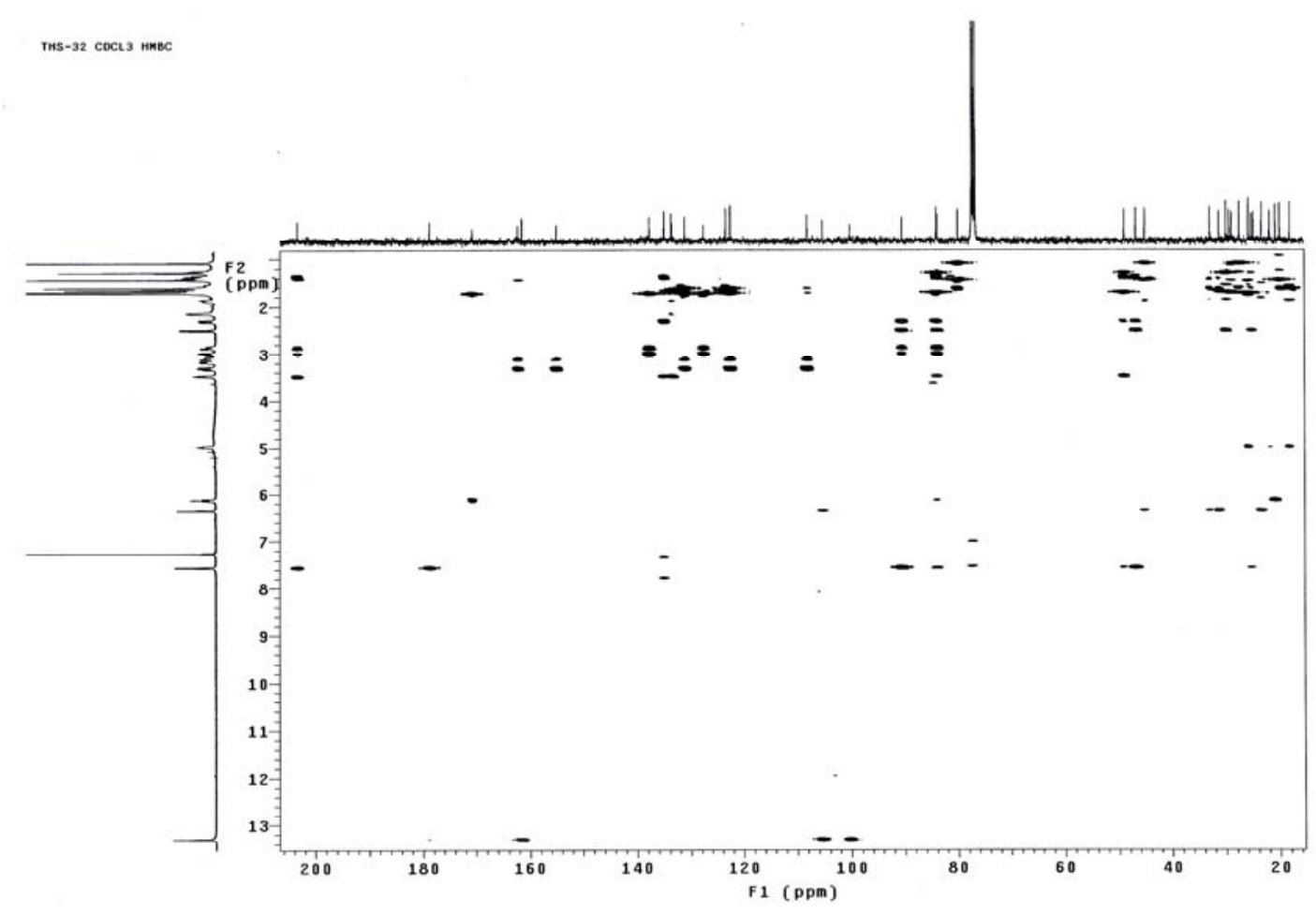


Figure S21. ${ }^{1} \mathrm{H}-{ }^{1} \mathrm{H}$ COSY spectrum (600 MHz, $\mathrm{CDCl}_{3}$ ) of gambogefic acid (4)

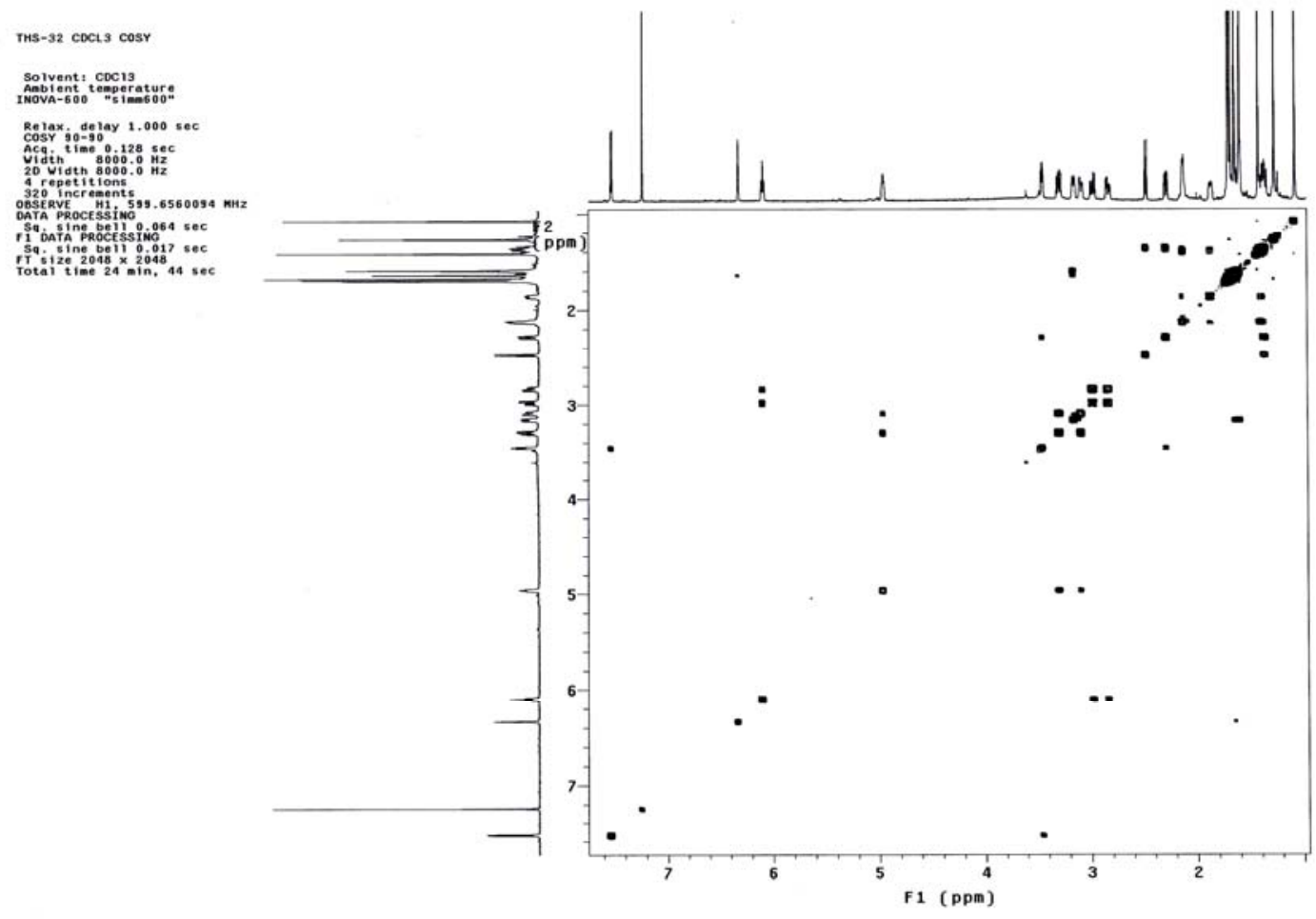

Figure S22. ${ }^{1} \mathrm{H}$ NMR spectrum (400 MHz, $\left.\mathrm{CDCl}_{3}\right)$ of 7-methoxygambogellic acid (5)

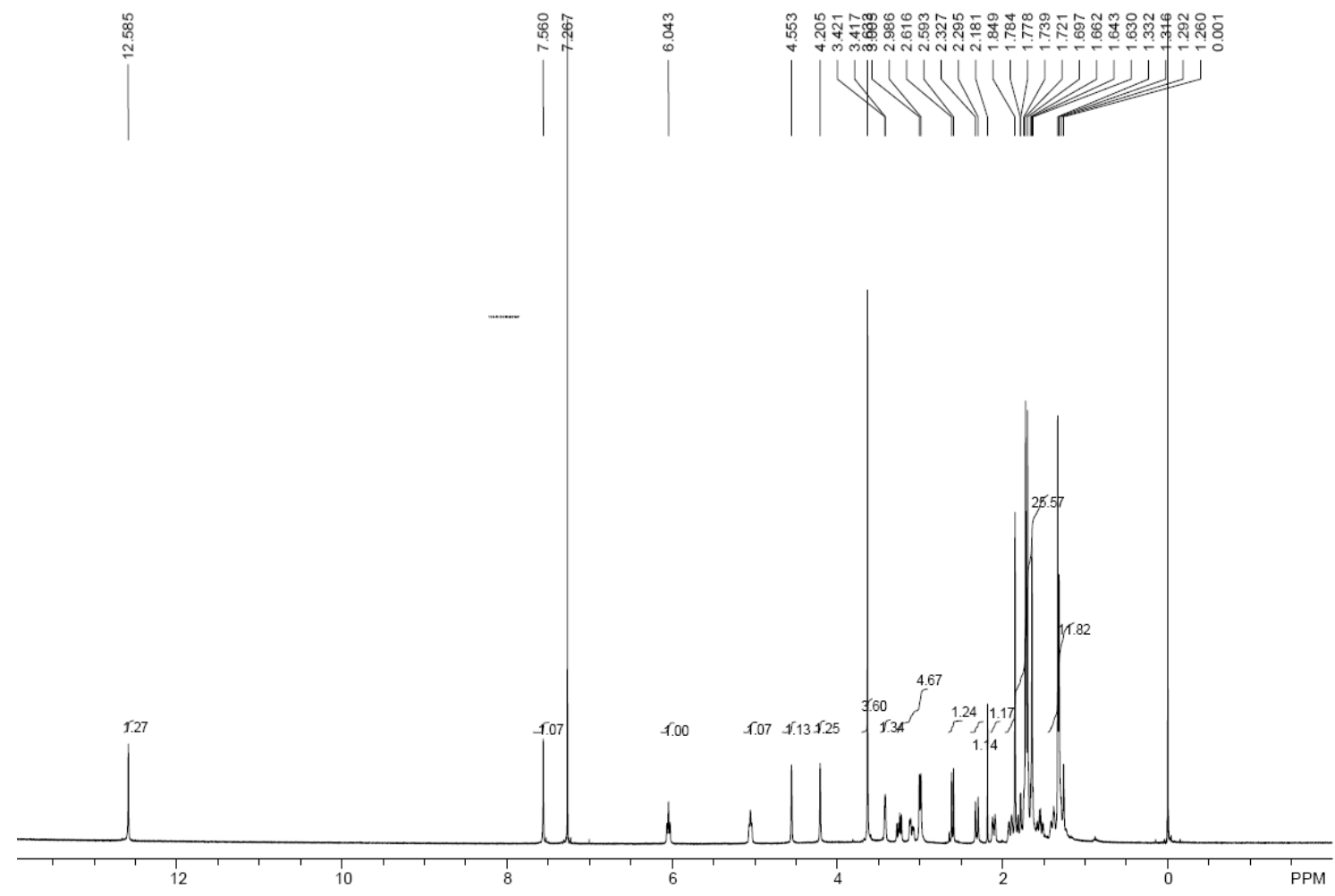


Figure S23. ${ }^{13} \mathrm{C}$ NMR spectrum (100 MHz, $\left.\mathrm{CDCl}_{3}\right)$ of 7-methoxygambogellic acid (5)

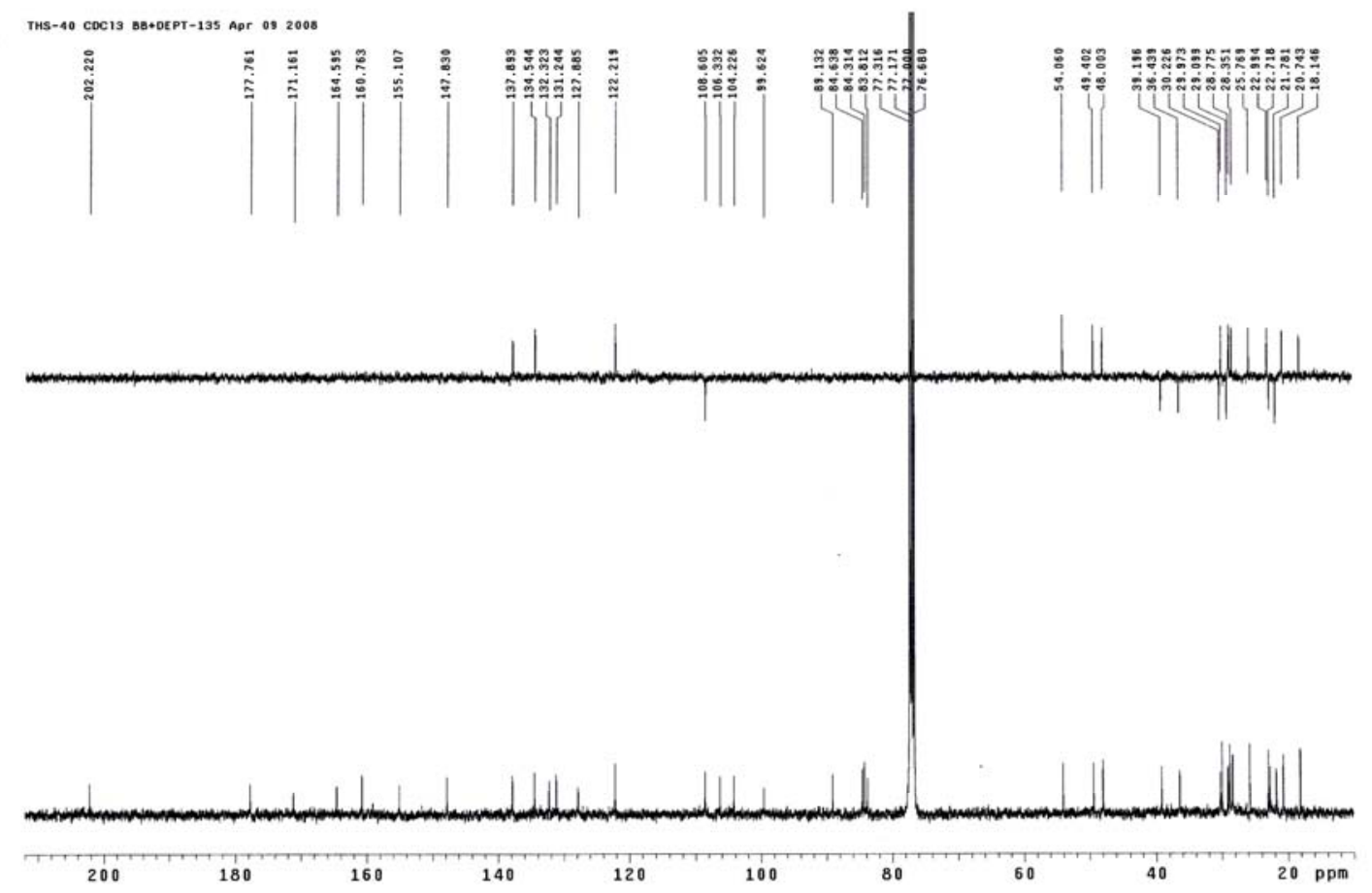

Figure S24. HSQC spectrum (100 MHz, $\mathrm{CDCl}_{3}$ ) of 7-methoxygambogellic acid (5)

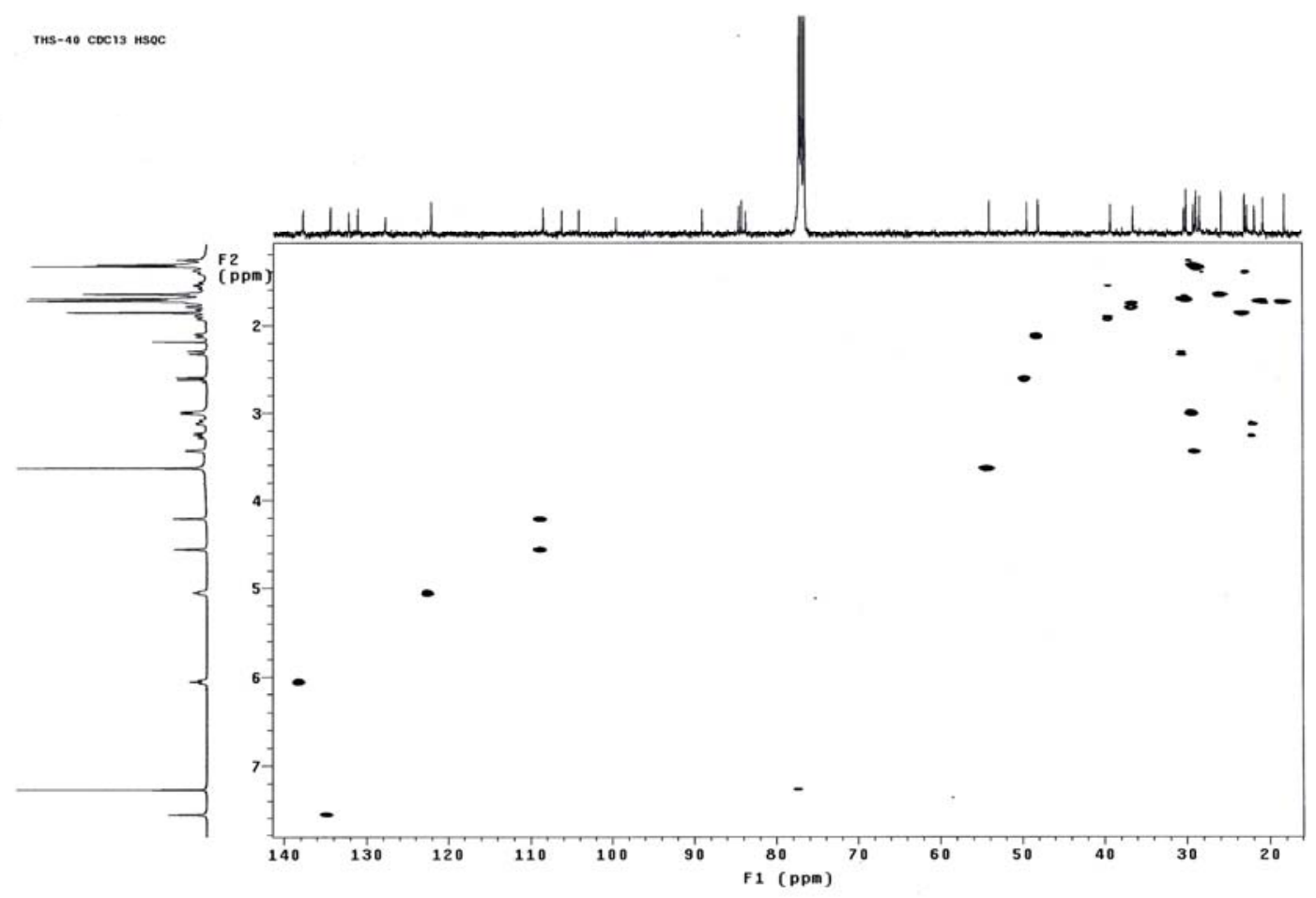


Figure S25. HMBC spectrum (100 MHz, $\mathrm{CDCl}_{3}$ ) of 7-methoxygambogellic acid (5)

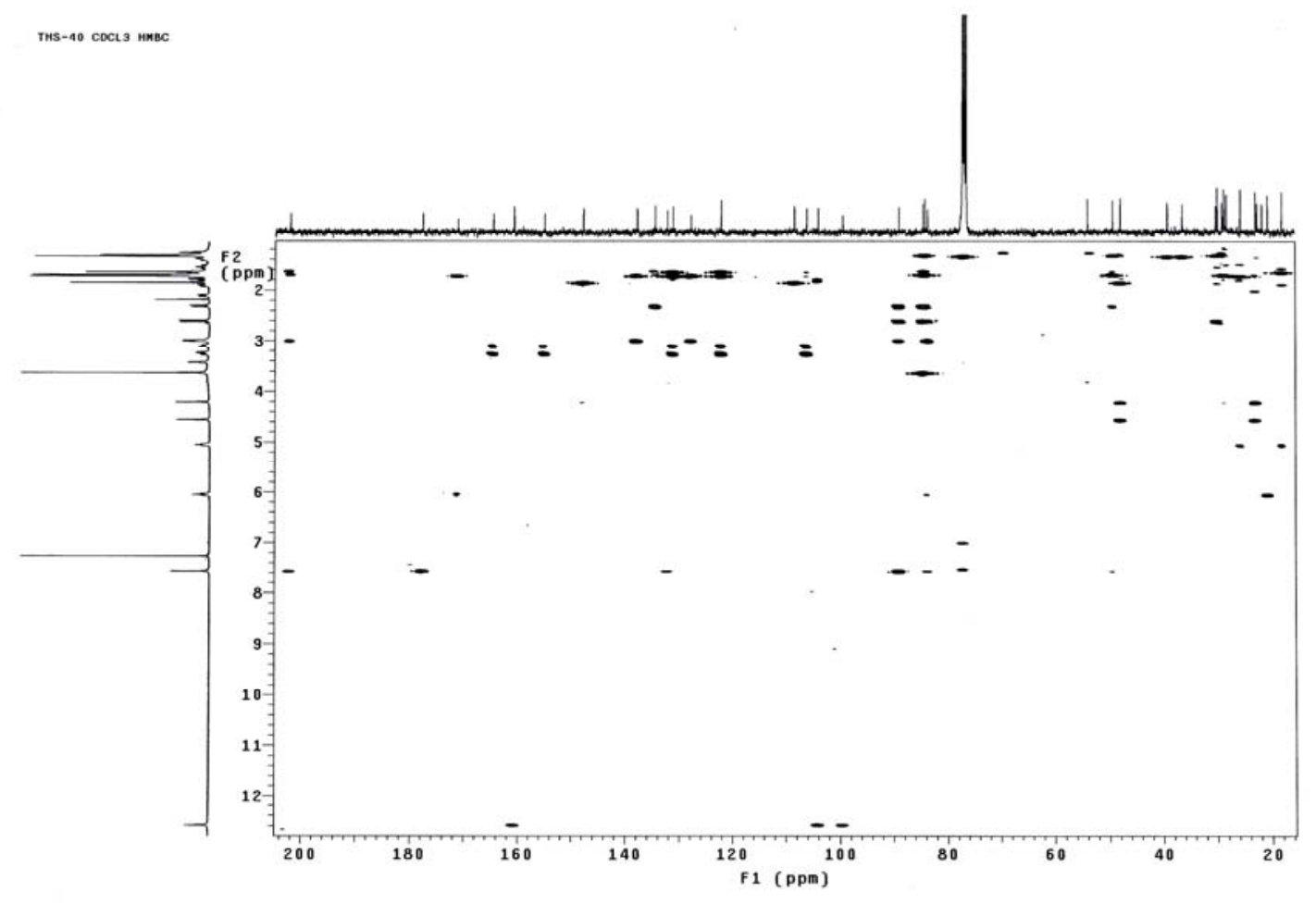

Figure S26. ${ }^{1} \mathrm{H}-{ }^{1} \mathrm{H}$ COSY spectrum (600 MHz, $\mathrm{CDCl}_{3}$ ) of 7-methoxygambogellic acid (5)

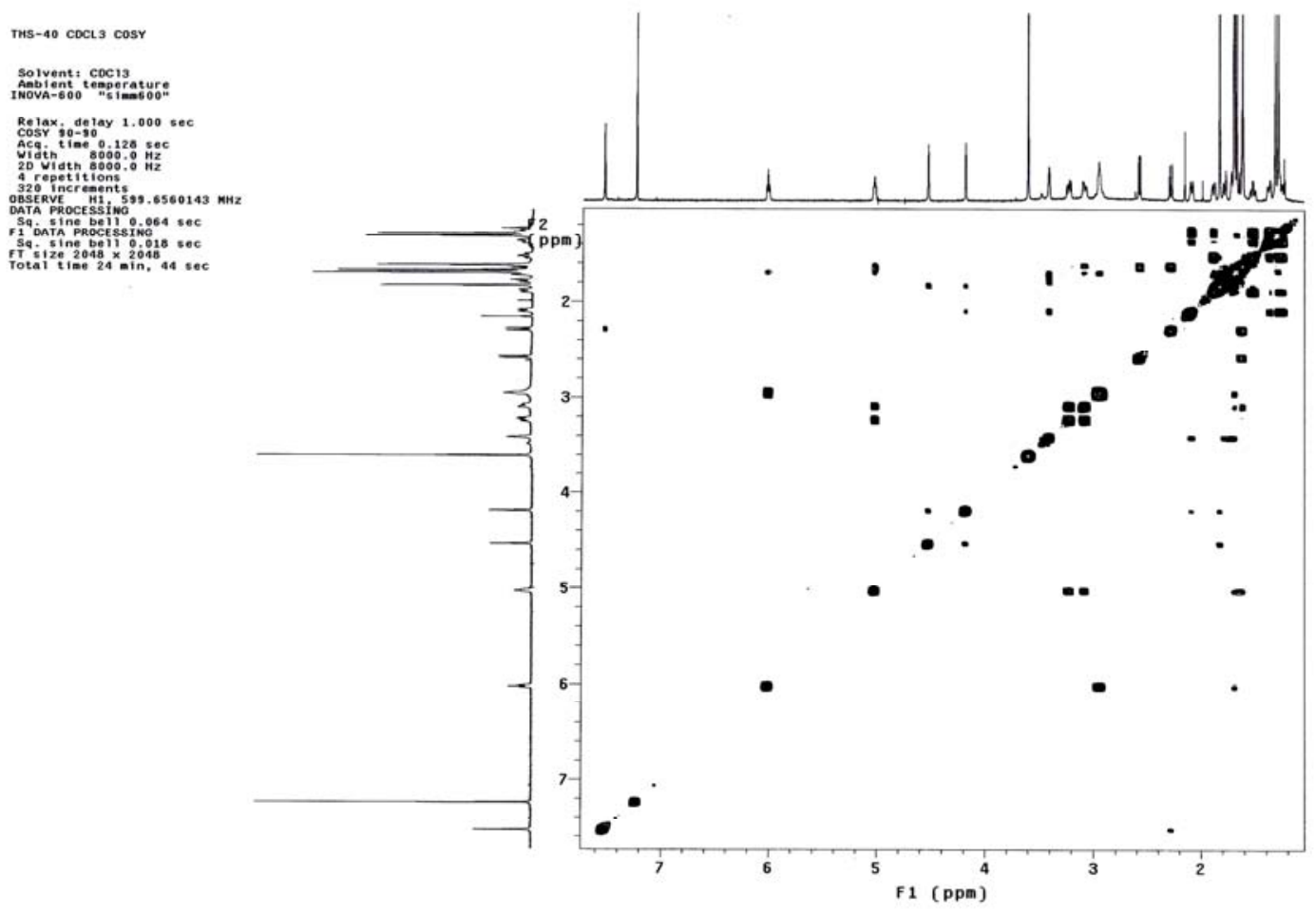


Figure S27. ROESY spectrum (600 MHz, $\mathrm{CDCl}_{3}$ ) of 7-methoxygambogellic acid (5)

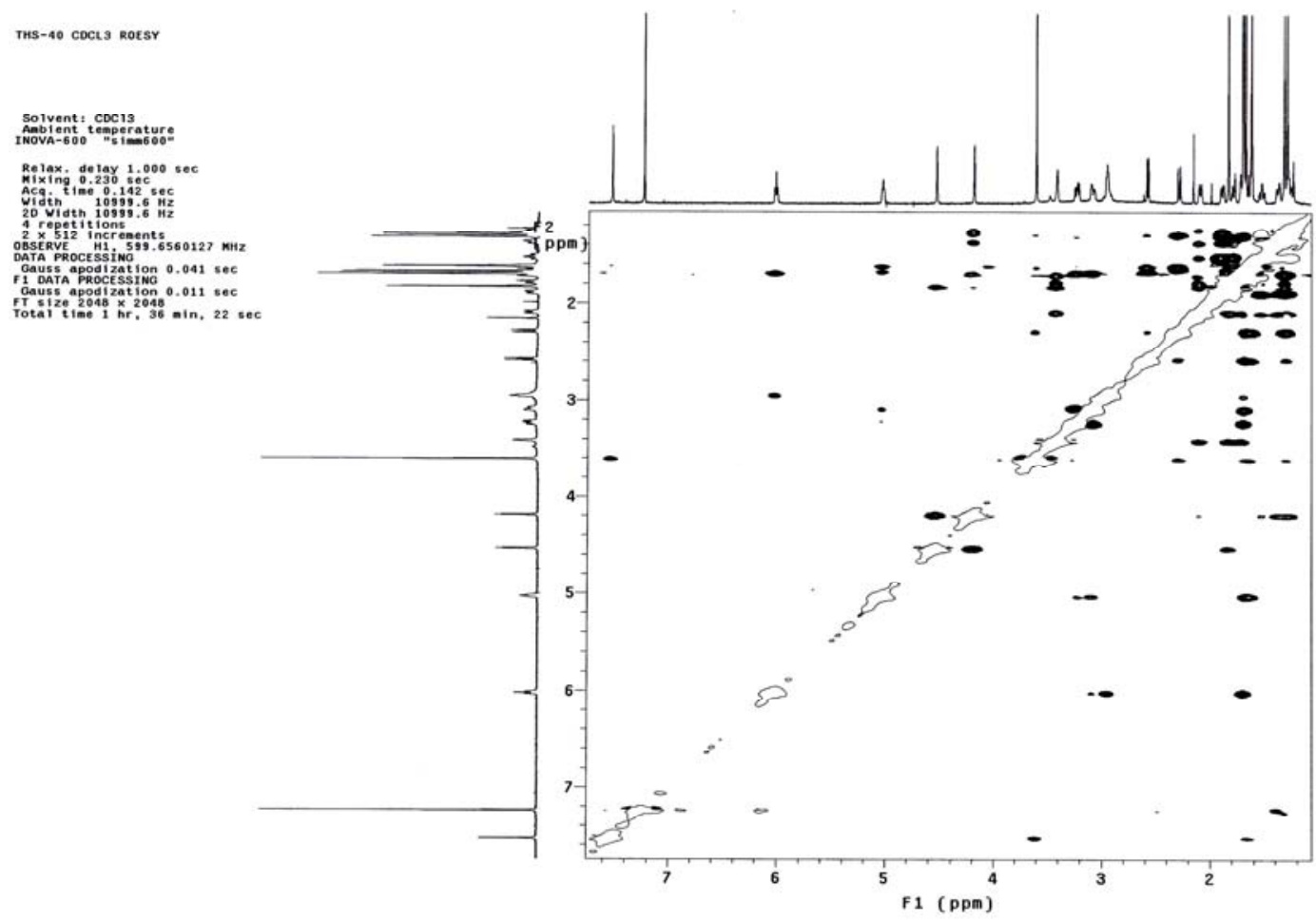

Figure S28. ${ }^{1} \mathrm{H}$ NMR spectrum (400 MHz, $\mathrm{CDCl}_{3}$ ) of 7-methoxygambogic acid (6)

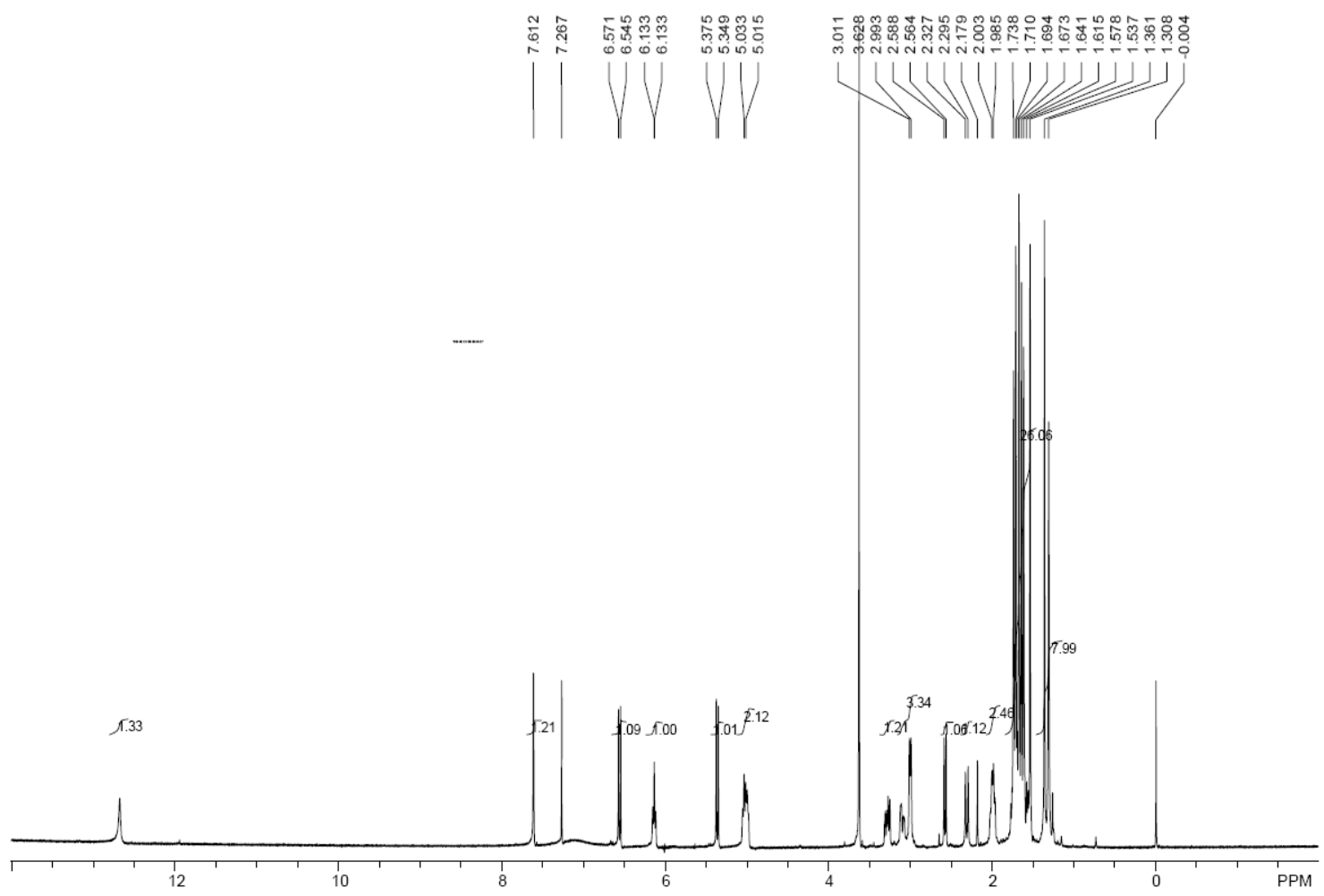


Figure S29. ${ }^{13} \mathrm{C}$ NMR spectrum (100 MHz, $\mathrm{CDCl}_{3}$ ) of 7-methoxygambogic acid (6)

THS-42 COCL3 BB+DEPT-135 Mar 142008
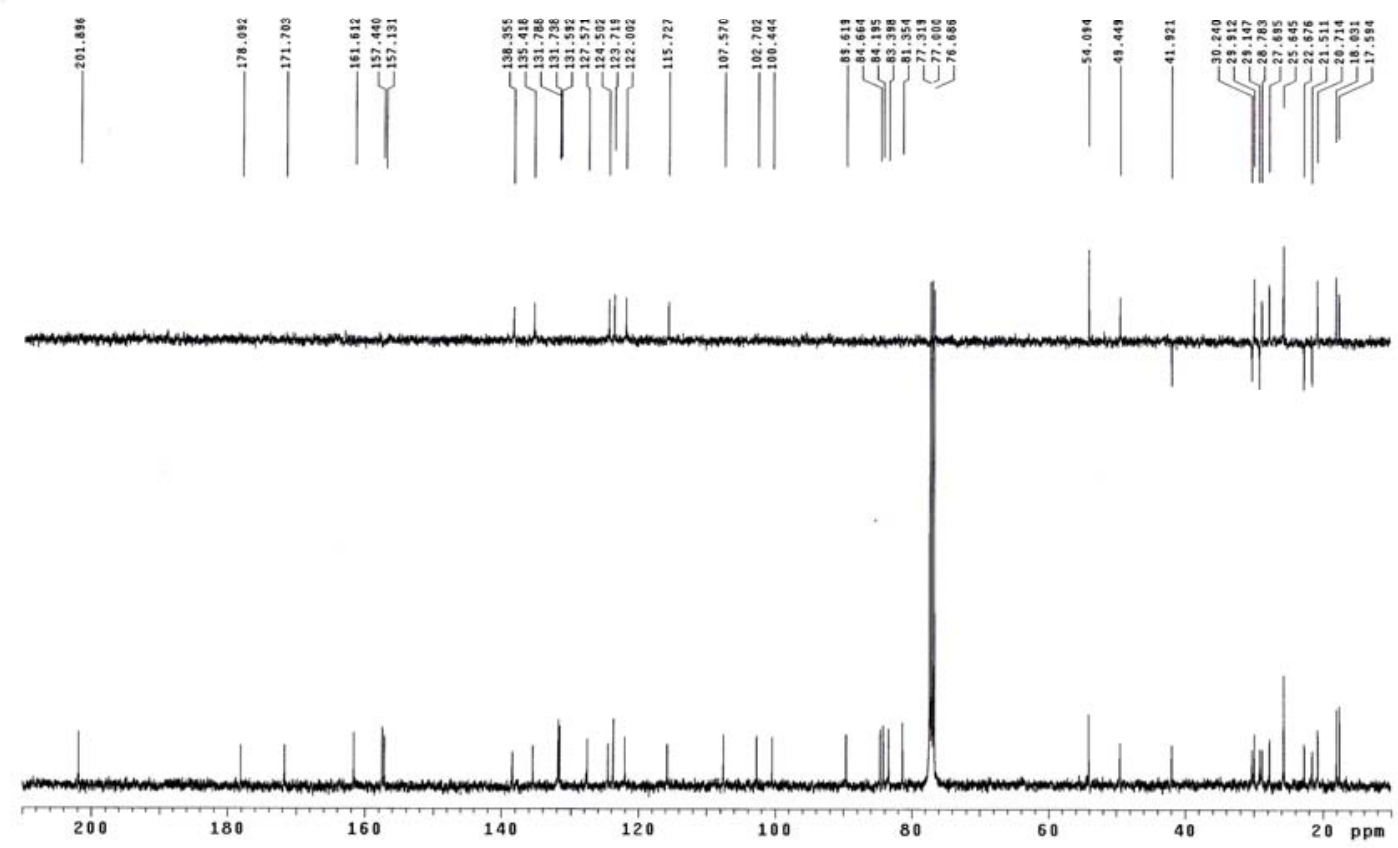

Figure S30. HSQC spectrum (100 MHz, $\mathrm{CDCl}_{3}$ ) of 7-methoxygambogic acid (6)

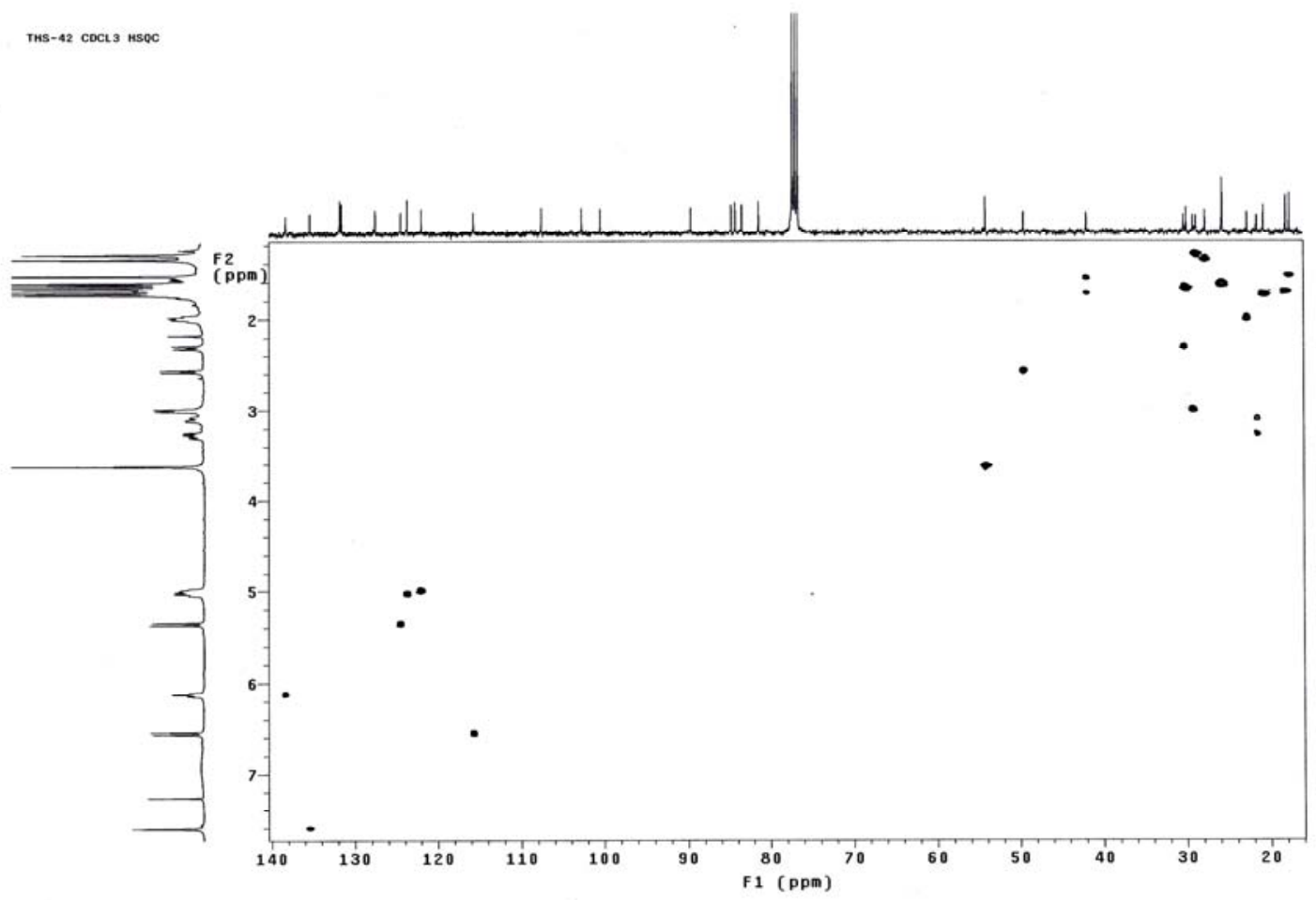


Figure S31. HMBC spectrum (100 MHz, $\left.\mathrm{CDCl}_{3}\right)$ of 7-methoxygambogic acid (6)

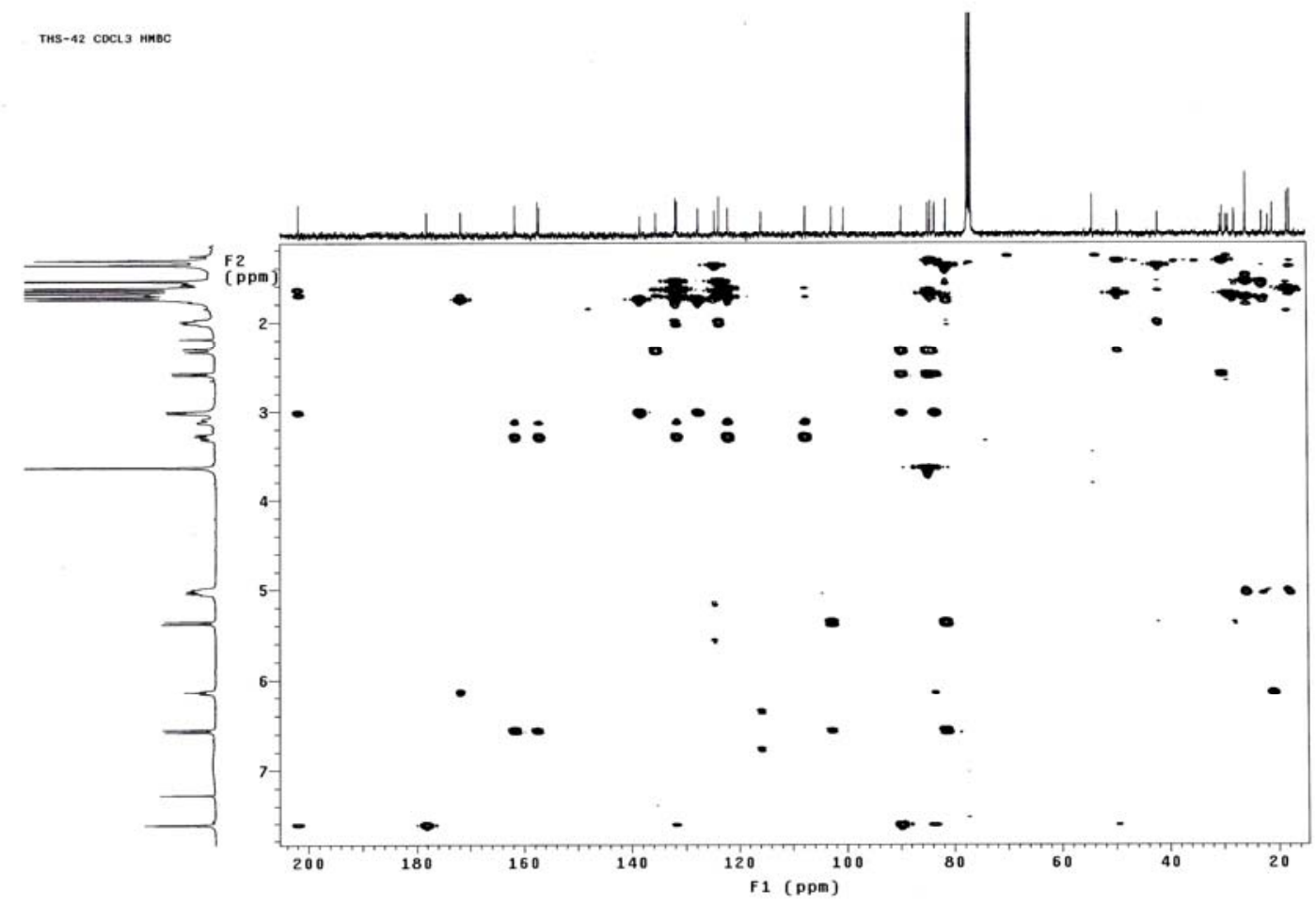

Figure S32. ${ }^{1} \mathrm{H}$ NMR spectrum $\left(400 \mathrm{MHz}, \mathrm{CDCl}_{3}\right)$ of 7-methoxyepigambogic acid (7)

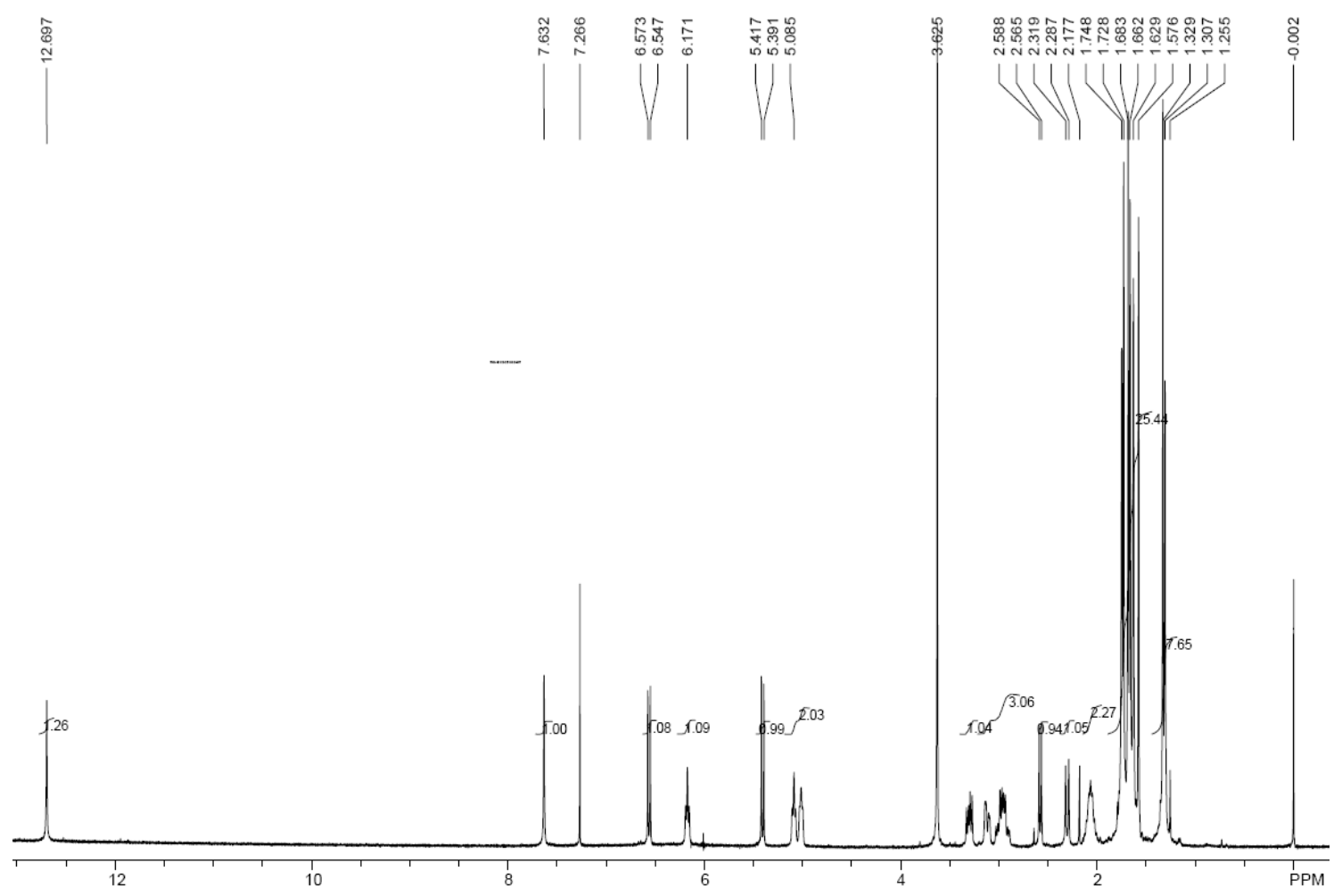


Figure S33. ${ }^{13} \mathrm{C}$ NMR spectrum $\left(100 \mathrm{MHz}, \mathrm{CDCl}_{3}\right)$ of 7-methoxyepigambogic acid (7)

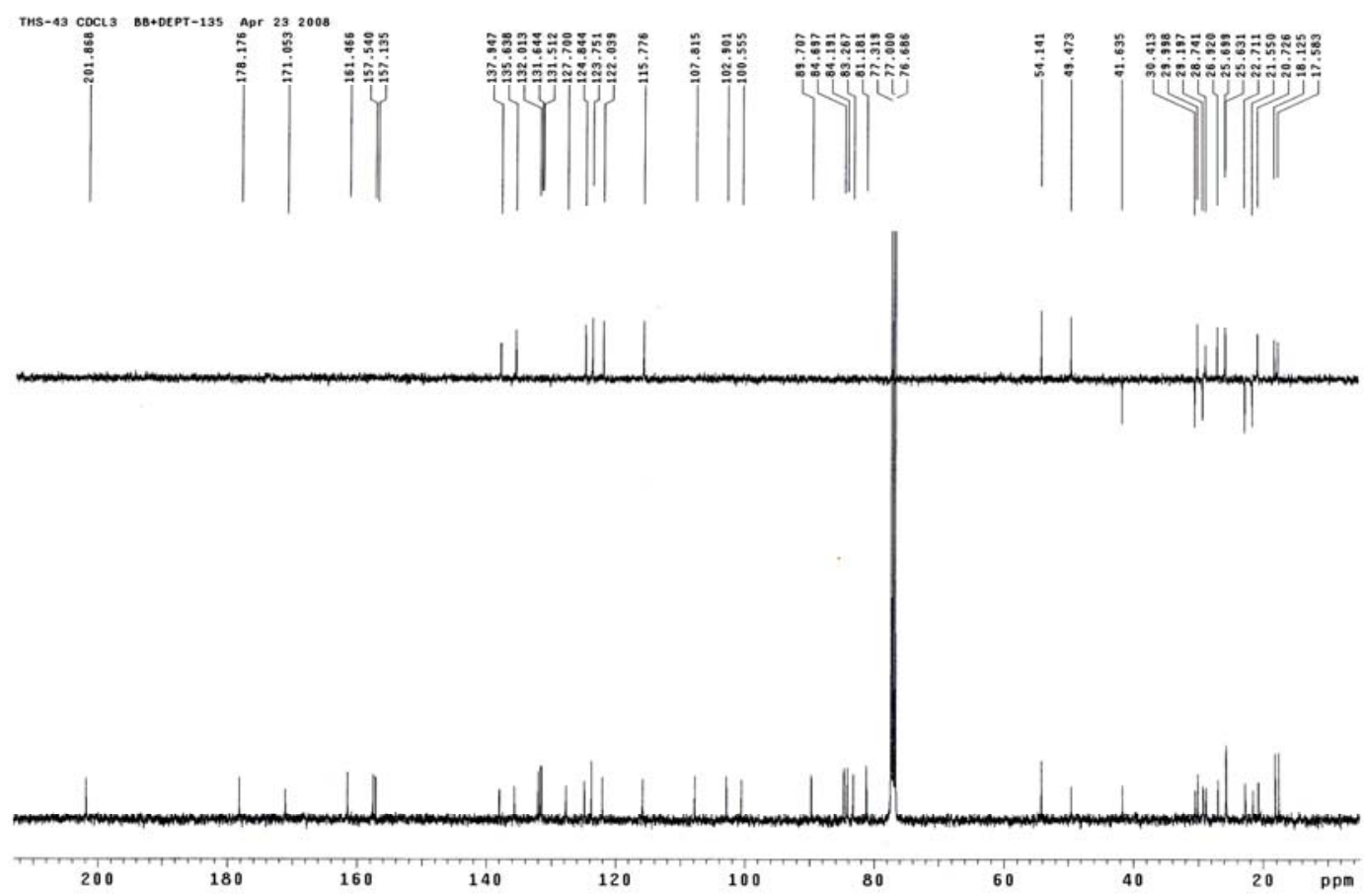

Figure S34. HMBC spectrum (100 MHz, $\mathrm{CDCl}_{3}$ ) of 7-methoxyepigambogic acid (7)

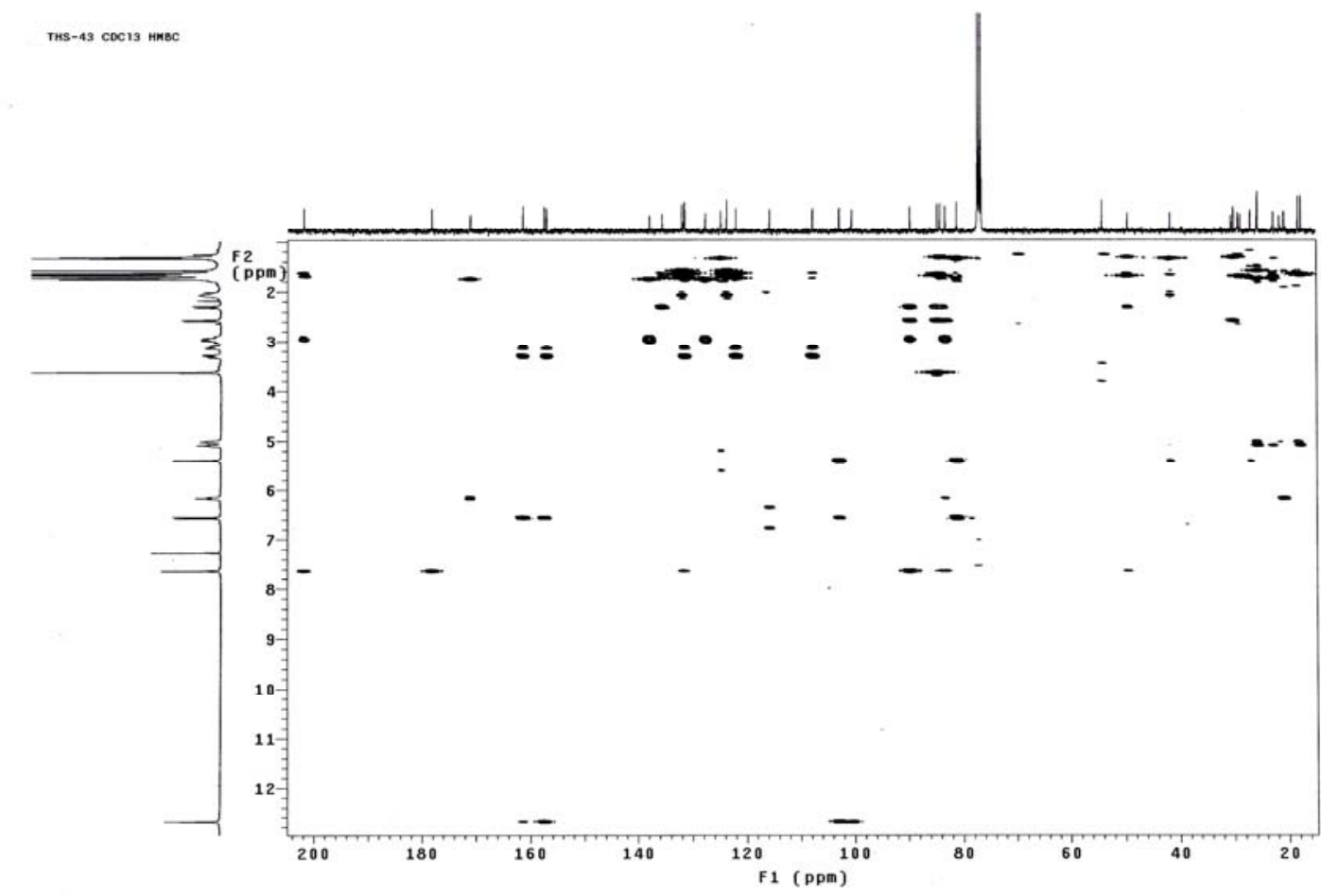


Figure S35. ${ }^{1} \mathrm{H}$ NMR spectrum (400 MHz, $\mathrm{CDCl}_{3}$ ) of 8,8a-dihydro-8-hydroxymorellic acid (8)

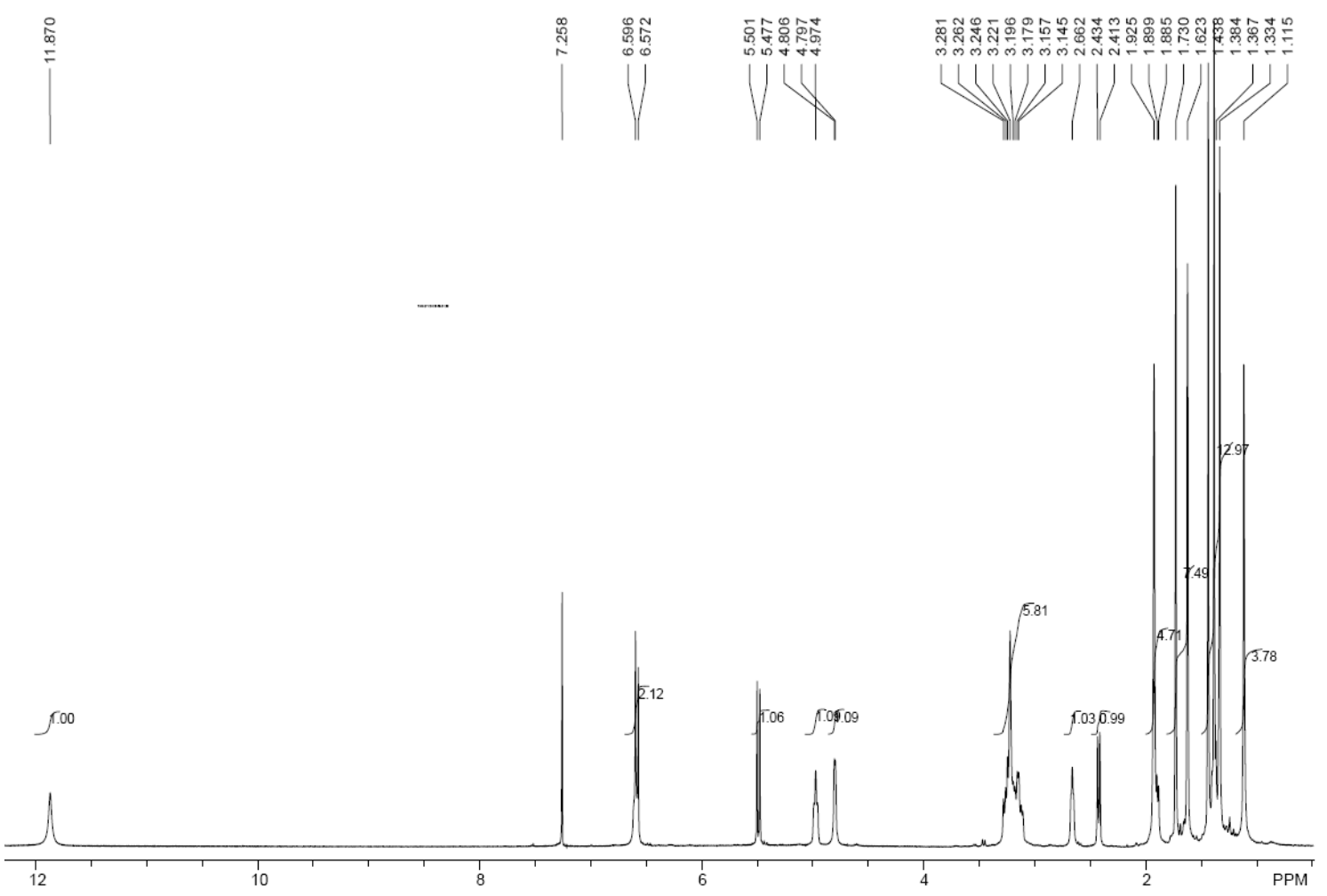

Figure S36. ${ }^{13} \mathrm{C}$ NMR spectrum (100 MHz, $\mathrm{CDCl}_{3}$ ) of 8,8a-dihydro-8-hydroxymorellic acid (8)

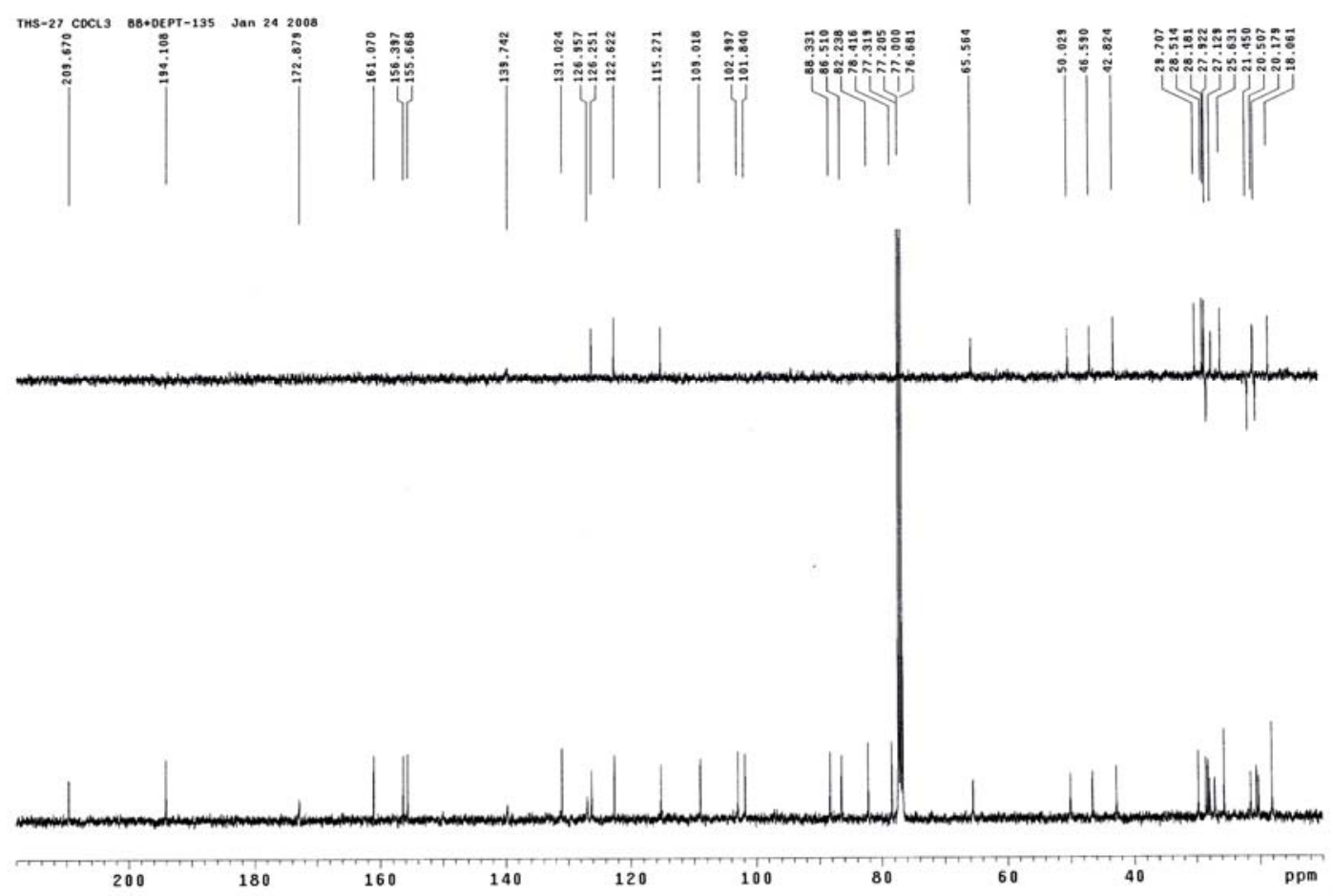


Figure S37. HSQC spectrum (100 MHz, $\mathrm{CDCl}_{3}$ ) of 8,8a-dihydro-8-hydroxymorellic acid (8)

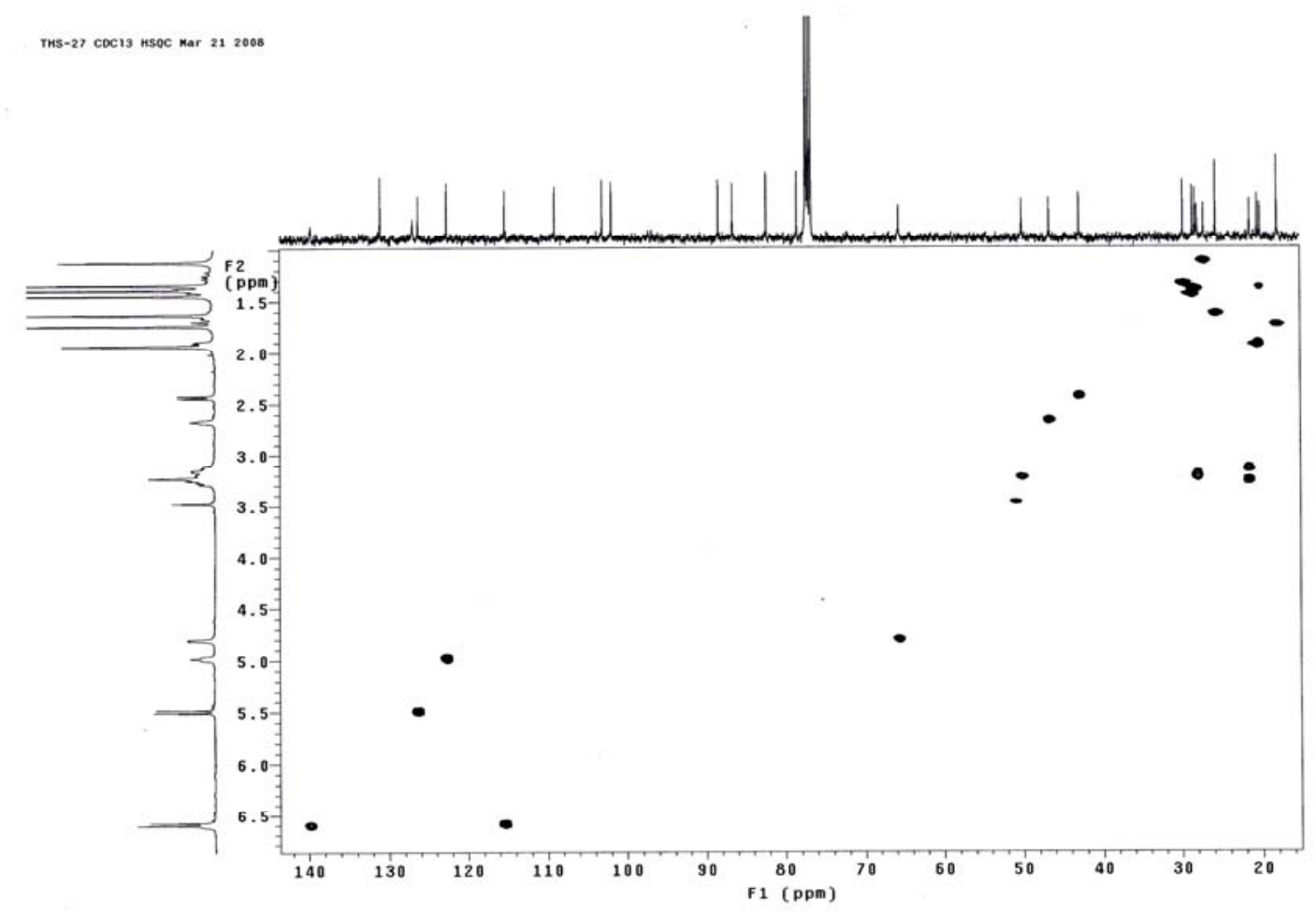

Figure S38. HMBC spectrum (100 MHz, $\left.\mathrm{CDCl}_{3}\right)$ of 8,8a-dihydro-8-hydroxymorellic acid (8)

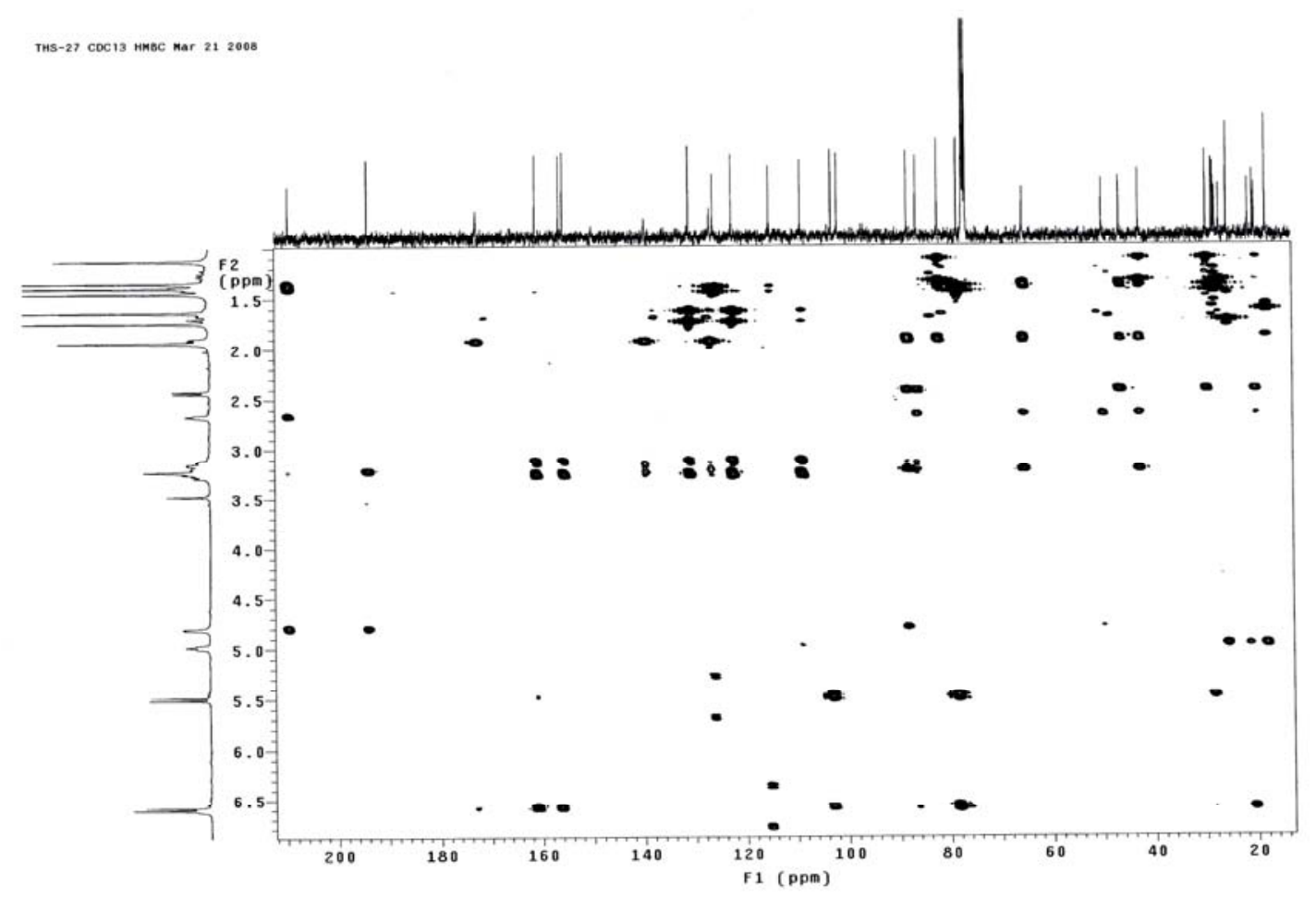


Figure S39. ROESY spectrum (600 MHz, $\mathrm{CDCl}_{3}$ ) of 8,8a-dihydro-8-hydroxymorellic acid (8)

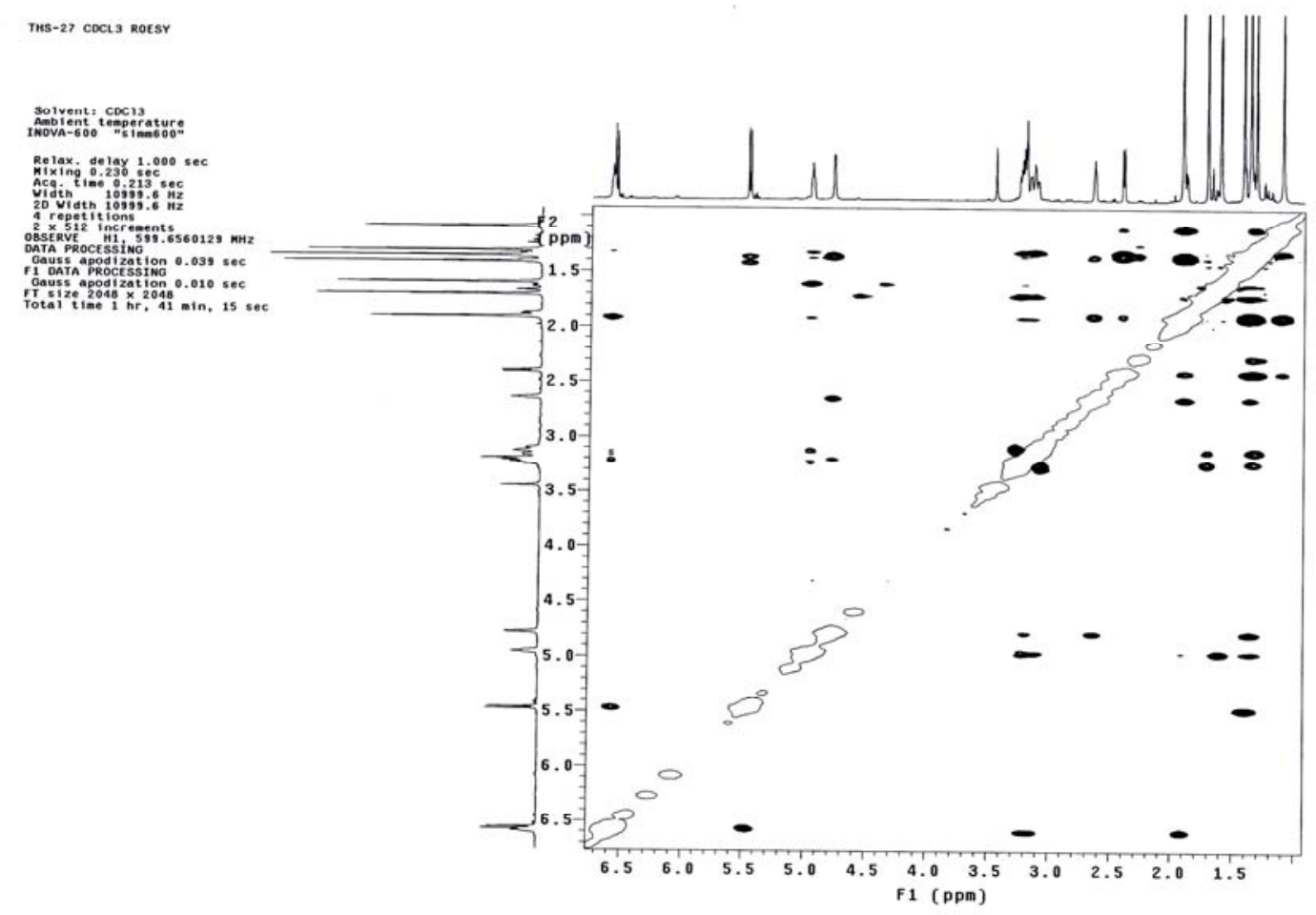

Figure S40. ${ }^{1} \mathrm{H}$ NMR spectrum (400 $\mathrm{MHz}, \mathrm{CDCl}_{3}$ ) of 8,8a-dihydro-8-hydroxygambogenic acid (9)

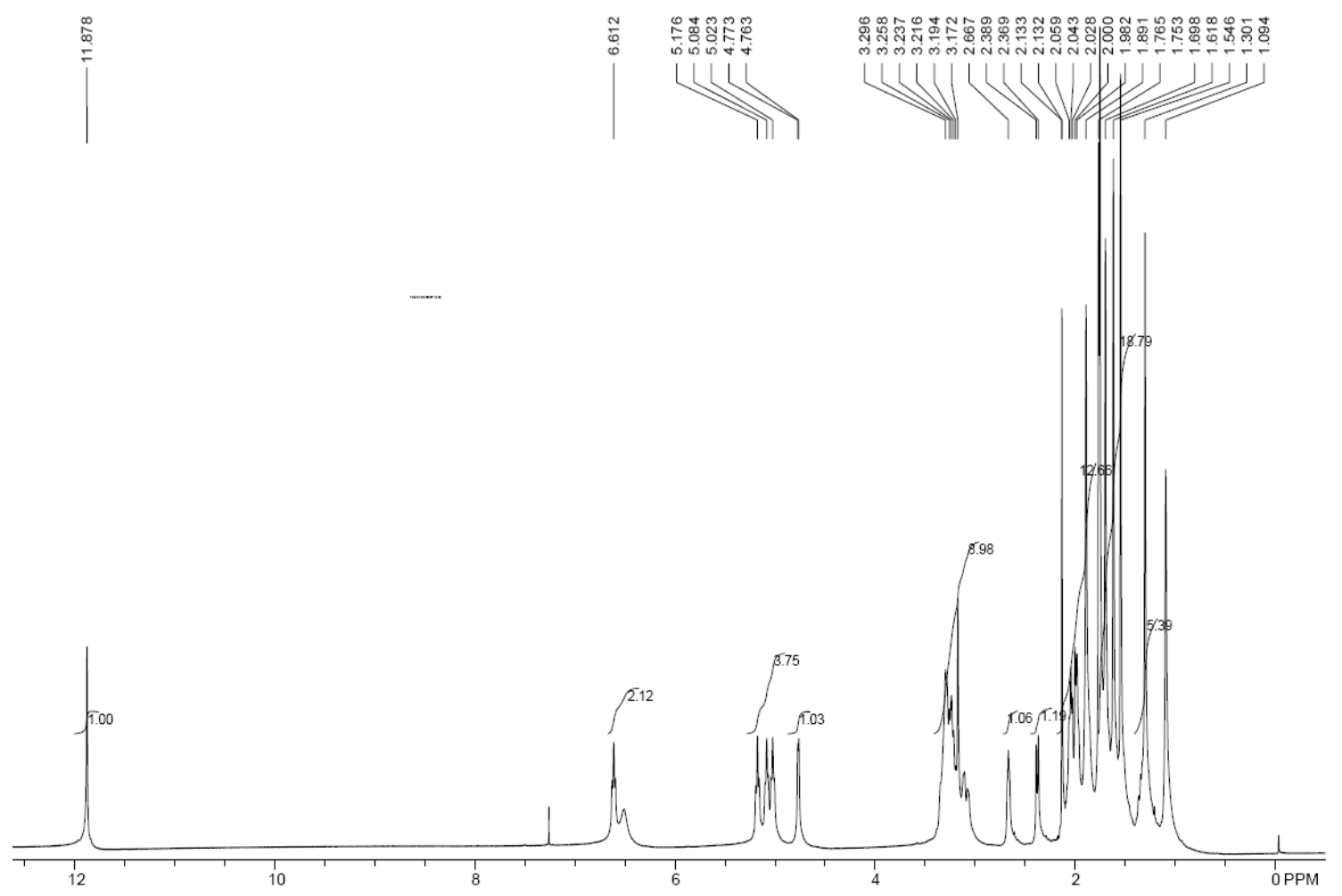


Figure S41. ${ }^{13} \mathrm{C}$ NMR spectrum $\left(100 \mathrm{MHz}, \mathrm{CDCl}_{3}\right)$ of 8,8a-dihydro-8-hydroxygambogenic acid (9)
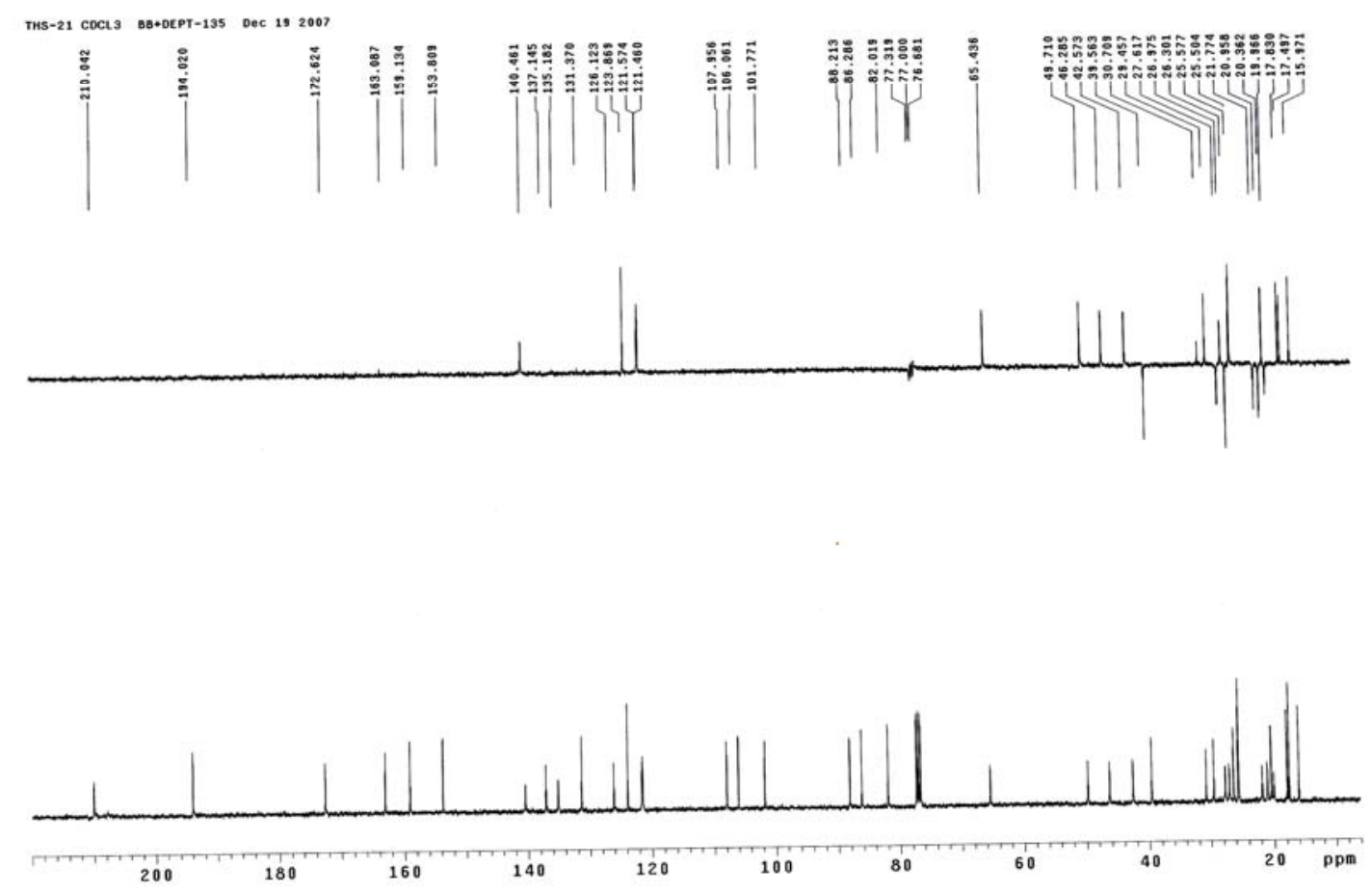

Figure S42. HSQC spectrum (100 MHz, $\mathrm{CDCl}_{3}$ ) of 8,8a-dihydro-8-hydroxygambogenic acid (9)

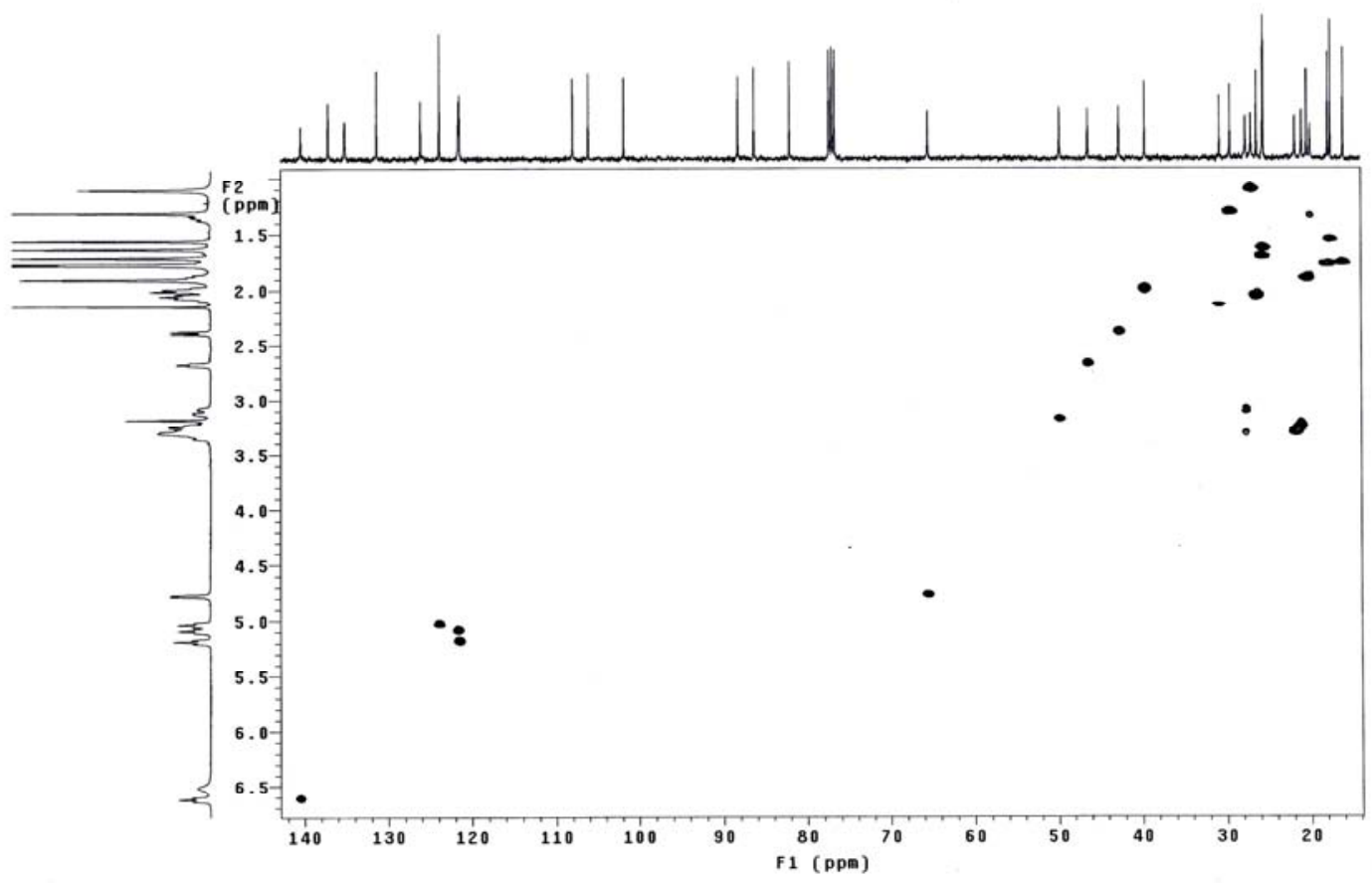


Figure S43. HMBC spectrum (100 MHz, $\mathrm{CDCl}_{3}$ ) of 8,8a-dihydro-8-hydroxygambogenic acid (9) THS-21 сосL3 нмвс

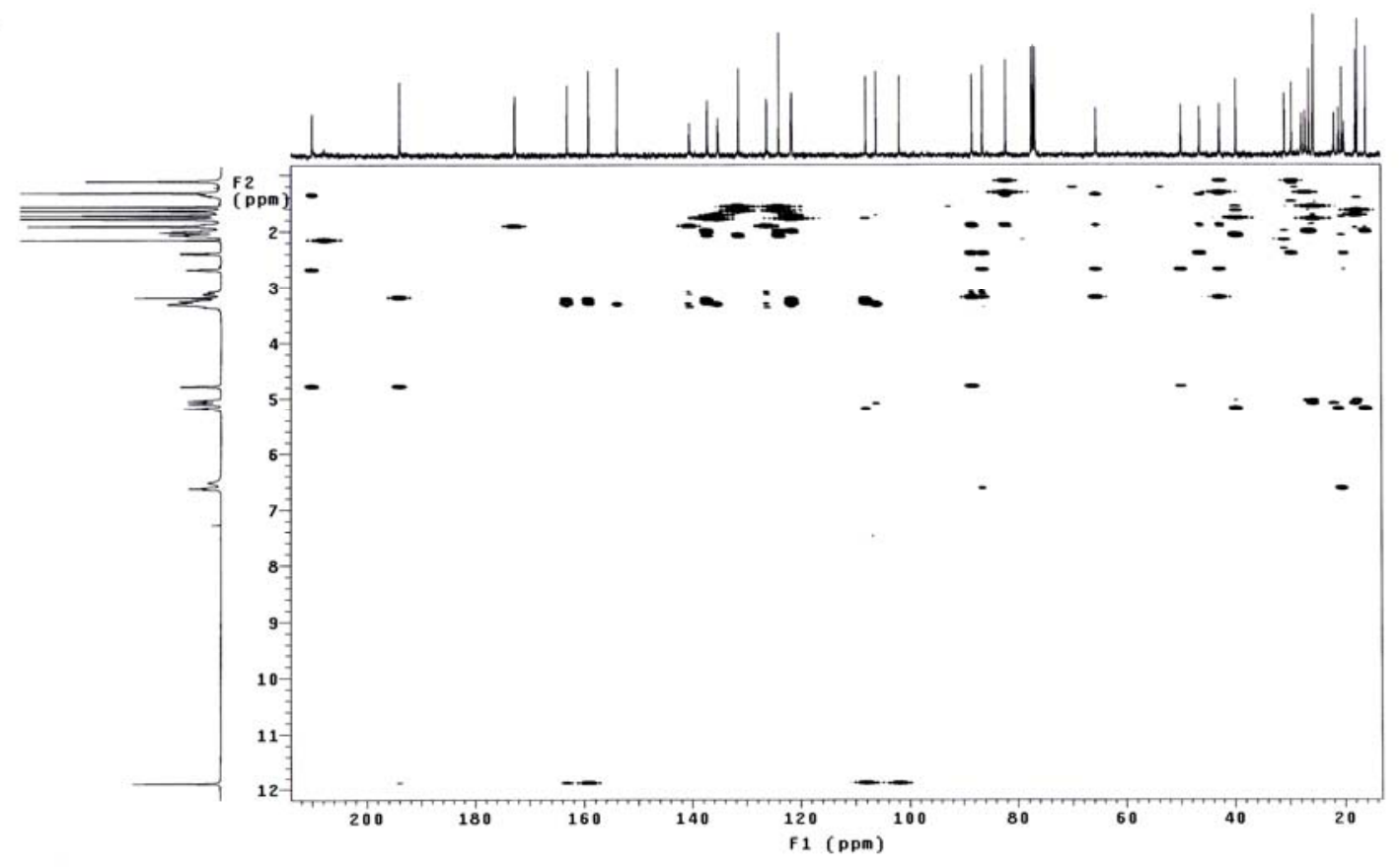

Figure S44. ROESY spectrum (600 MHz, $\mathrm{CDCl}_{3}$ ) of 8,8a-dihydro-8-hydroxygambogenic acid (9)

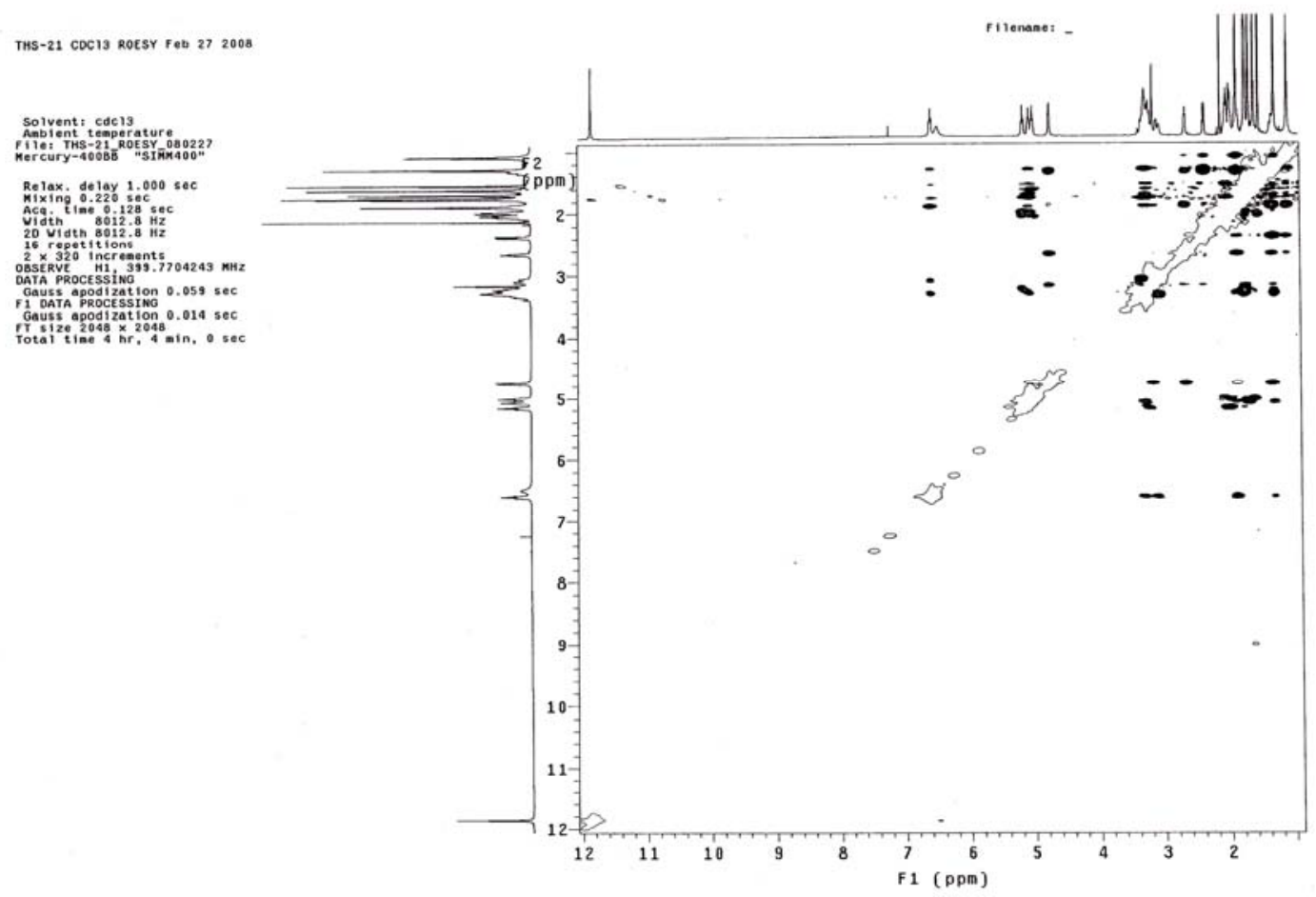


Figure S45. ${ }^{1} \mathrm{H}$ NMR spectrum (400 MHz, $\mathrm{CDCl}_{3}$ ) of oxygambogic acid (10)

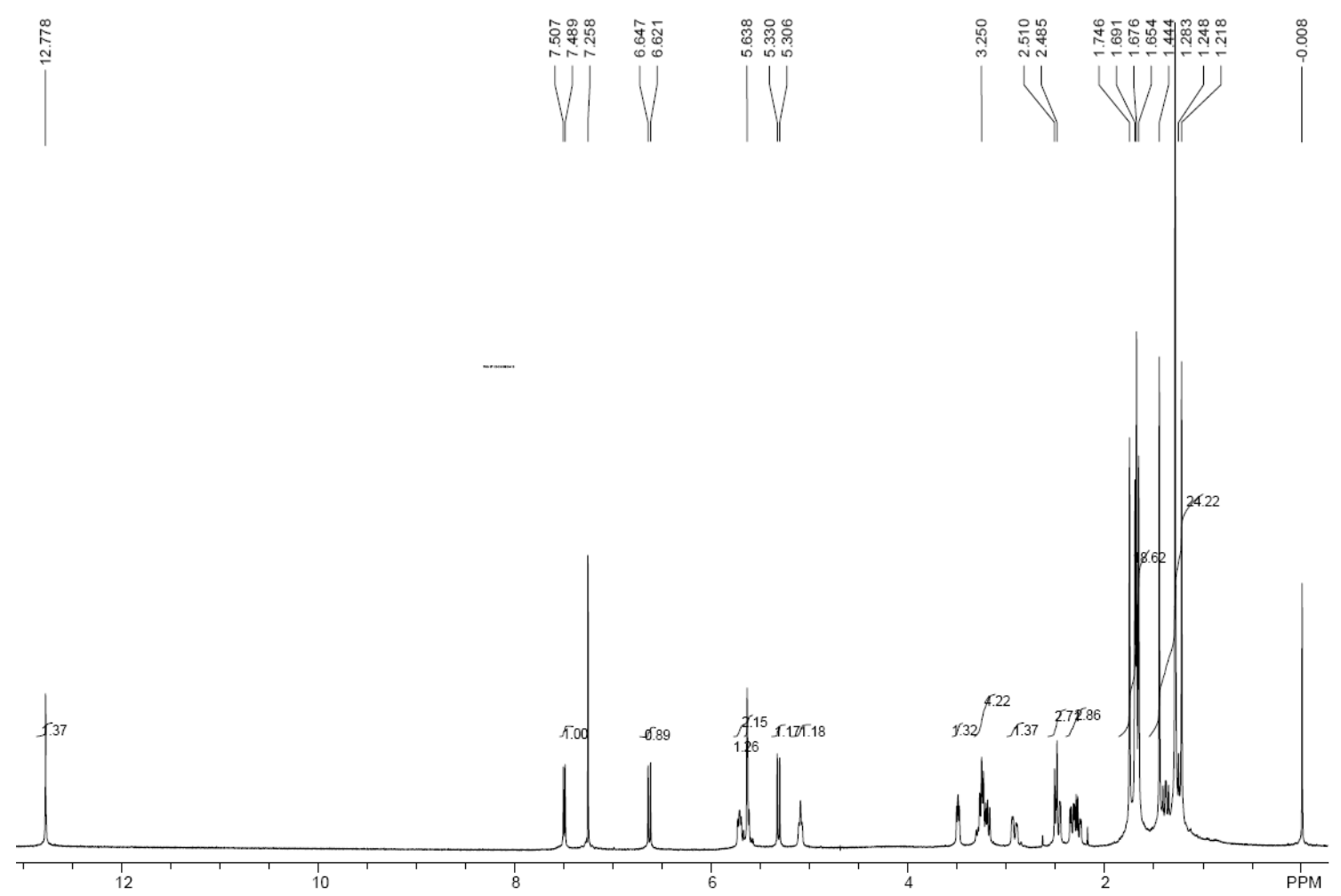

Figure S46. ${ }^{13} \mathrm{C}$ NMR spectrum $\left(100 \mathrm{MHz}, \mathrm{CDCl}_{3}\right)$ of oxygambogic acid (10)
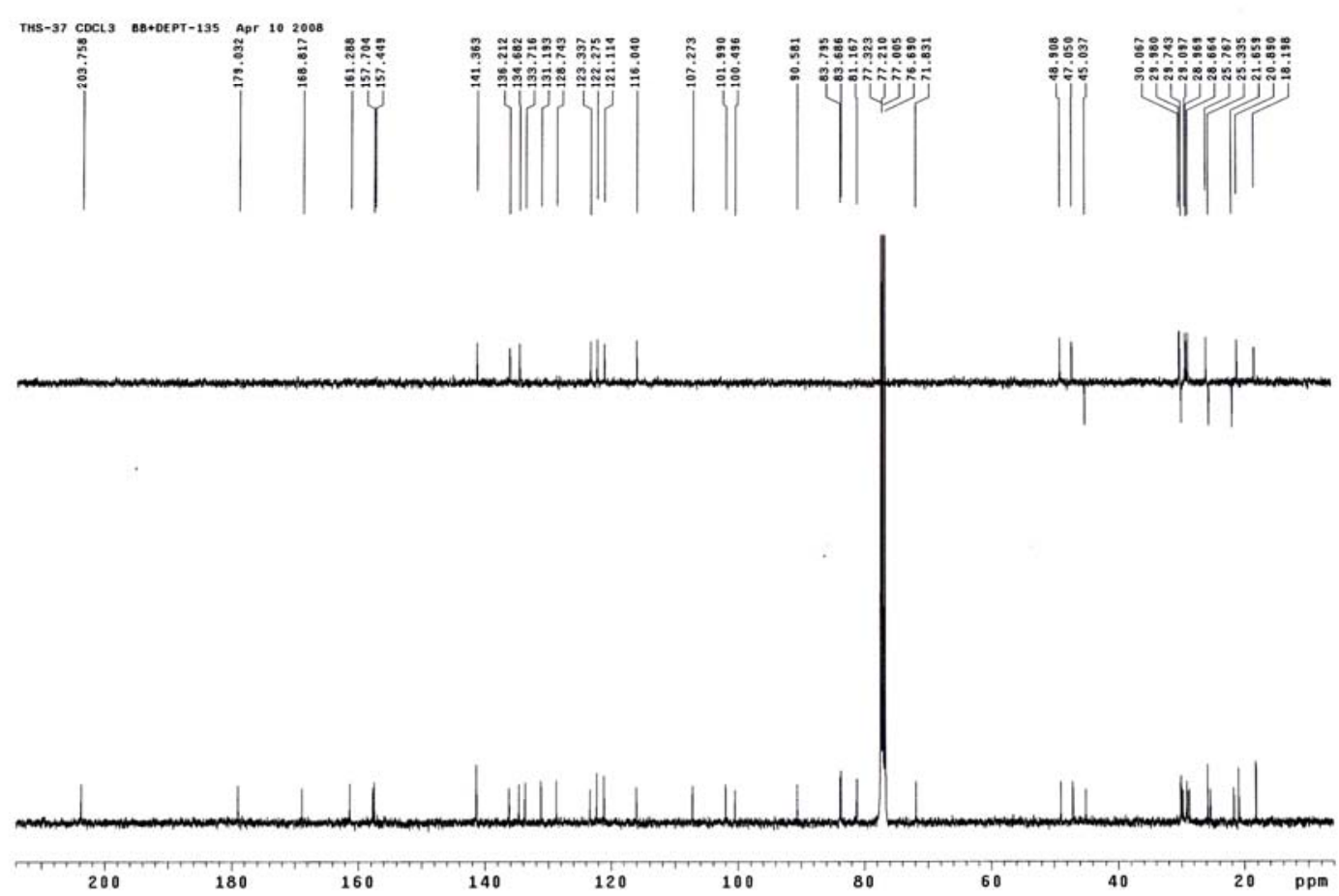
Figure S47. HSQC spectrum (100 MHz, $\mathrm{CDCl}_{3}$ ) of oxygambogic acid (10)

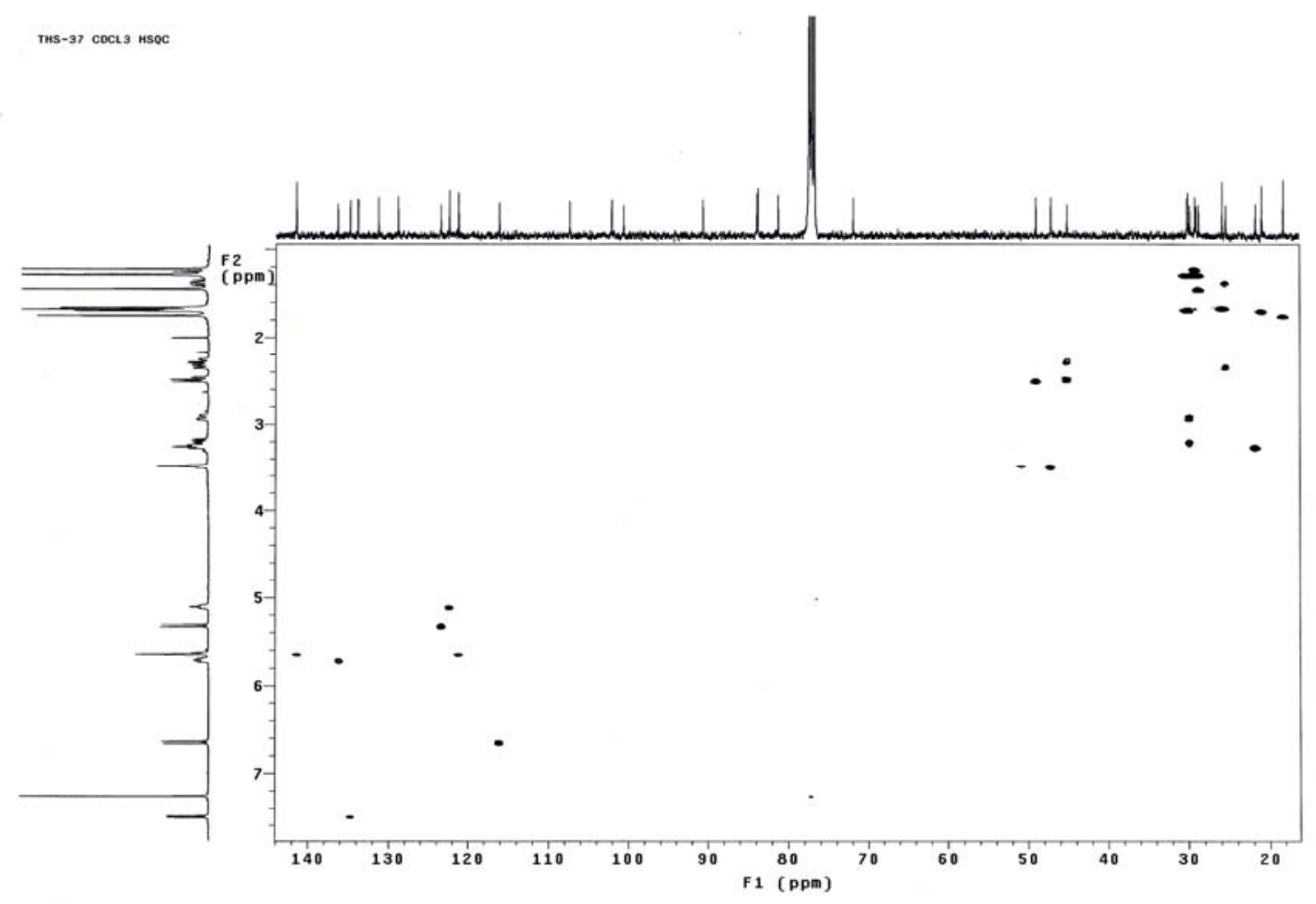

Figure S48. HMBC spectrum (100 MHz, $\mathrm{CDCl}_{3}$ ) of oxygambogic acid (10)

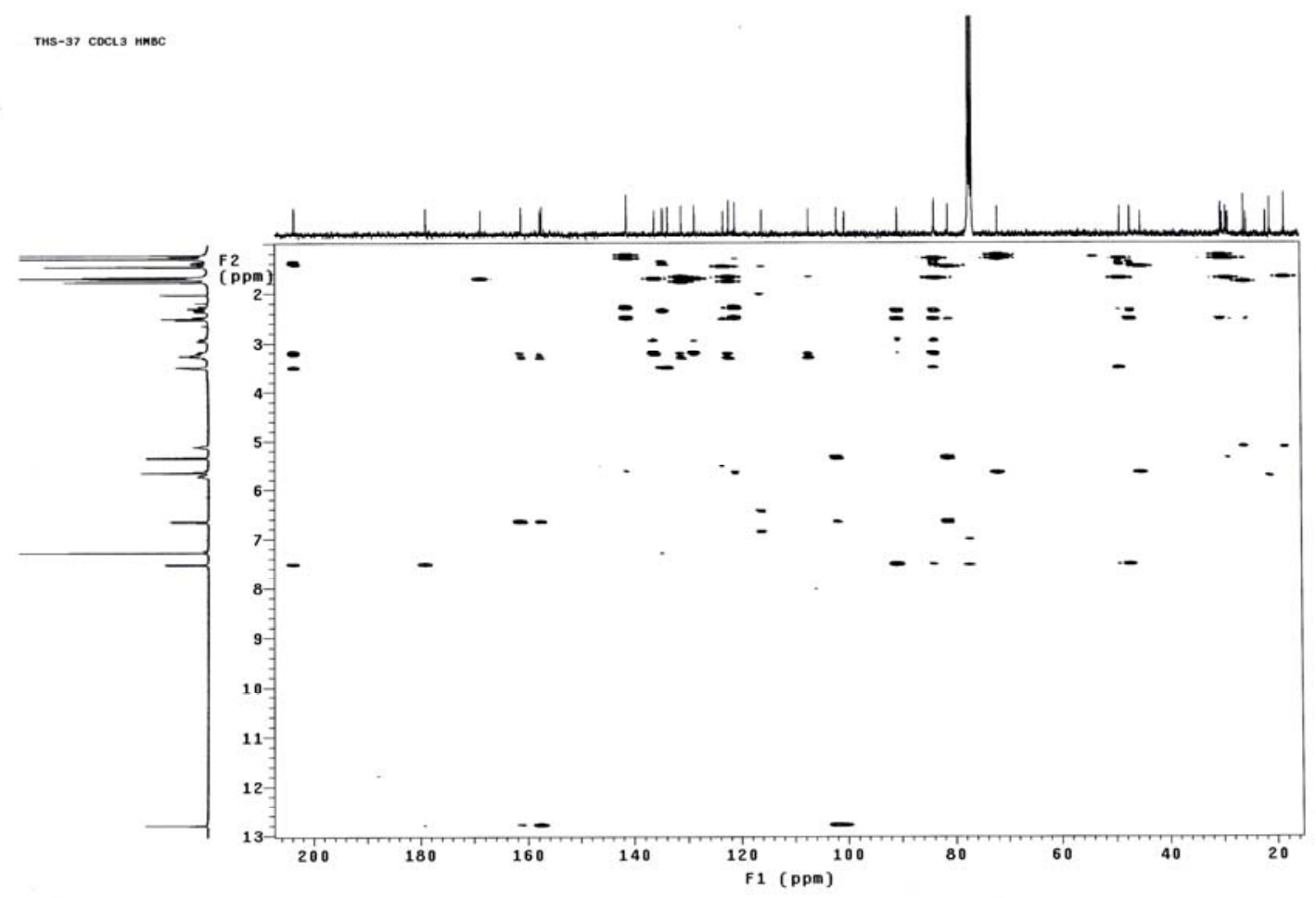


Figure S49. ROESY spectrum (600 MHz, $\mathrm{CDCl}_{3}$ ) of oxygambogic acid (10)

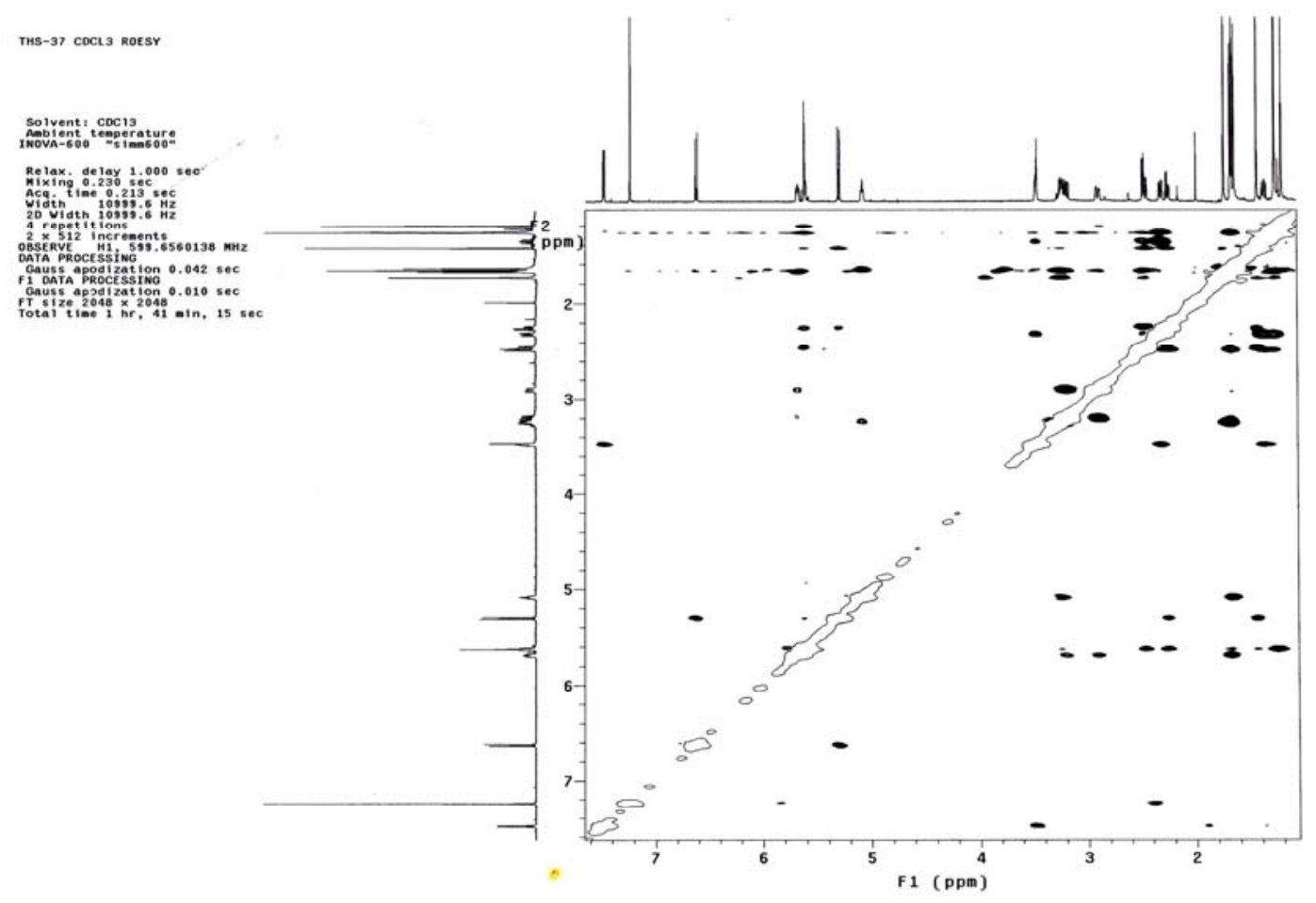

Figure S50. ${ }^{1} \mathrm{H}$ NMR spectrum (400 MHz, $\mathrm{CDCl}_{3}$ ) of gambogenific acid (11)

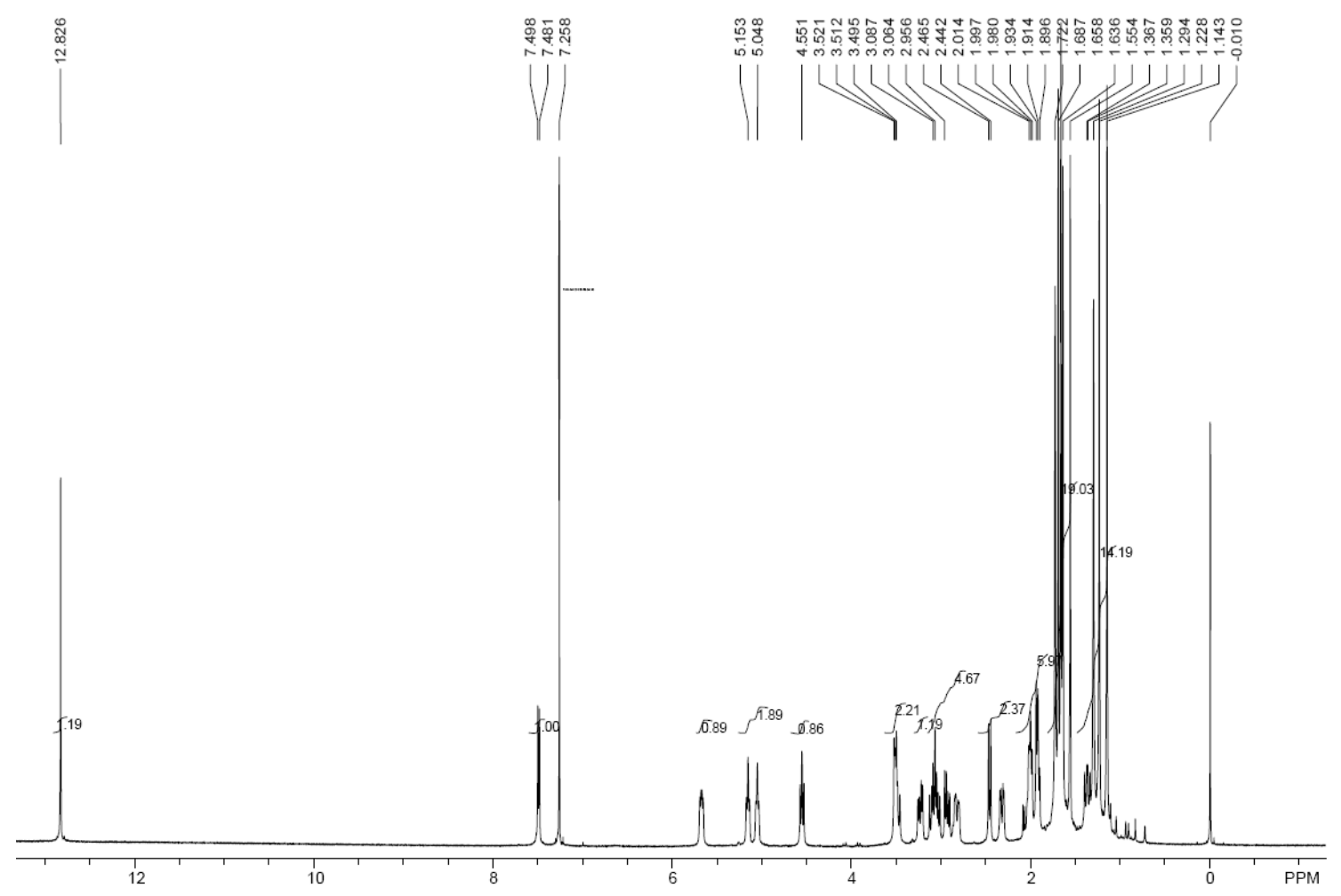


Figure S51. ${ }^{13} \mathrm{C}$ NMR spectrum $\left(100 \mathrm{MHz}, \mathrm{CDCl}_{3}\right)$ of gambogenific acid (11)

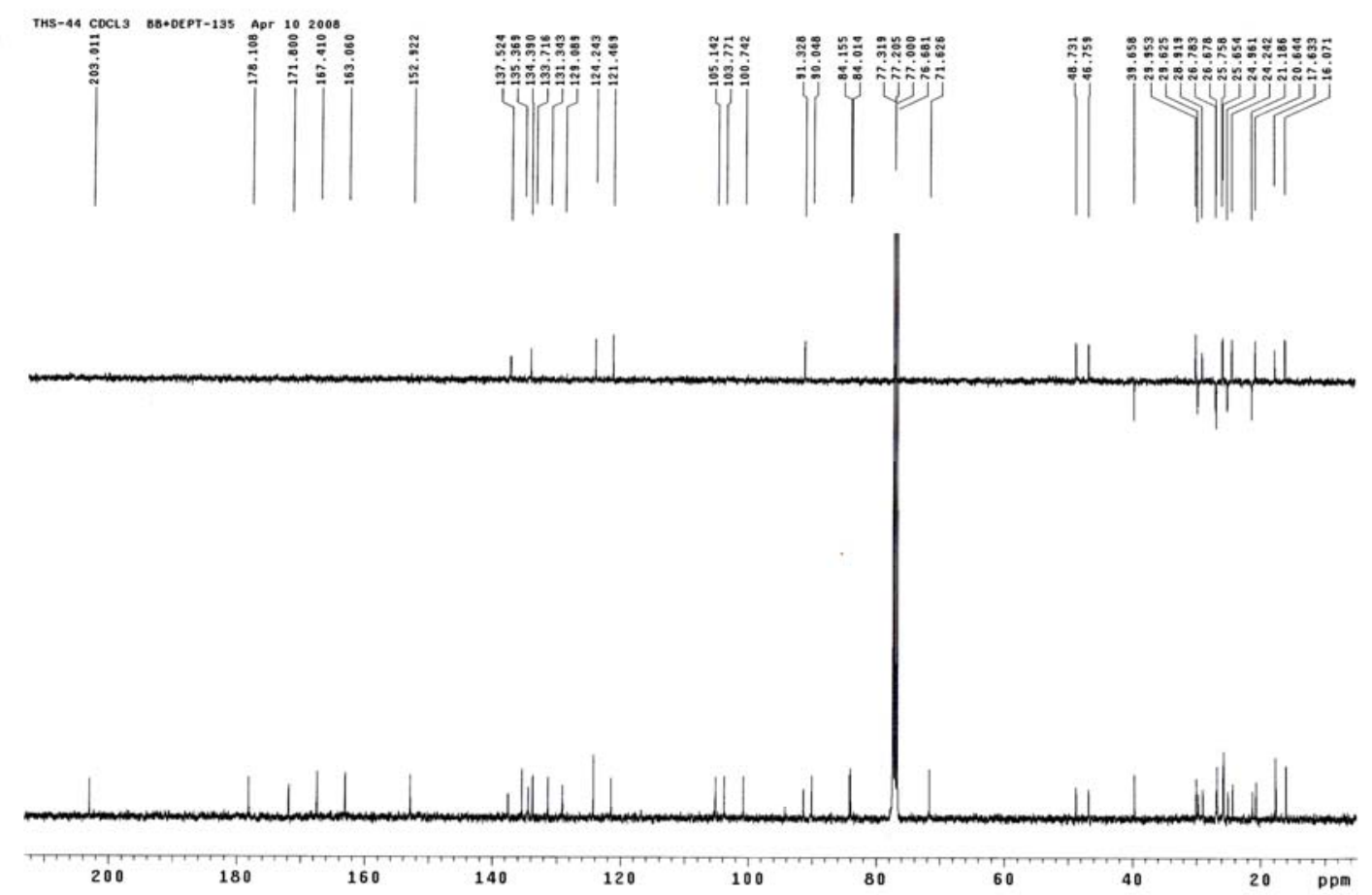

Figure S52. HSQC spectrum (100 MHz, $\mathrm{CDCl}_{3}$ ) of gambogenific acid (11)

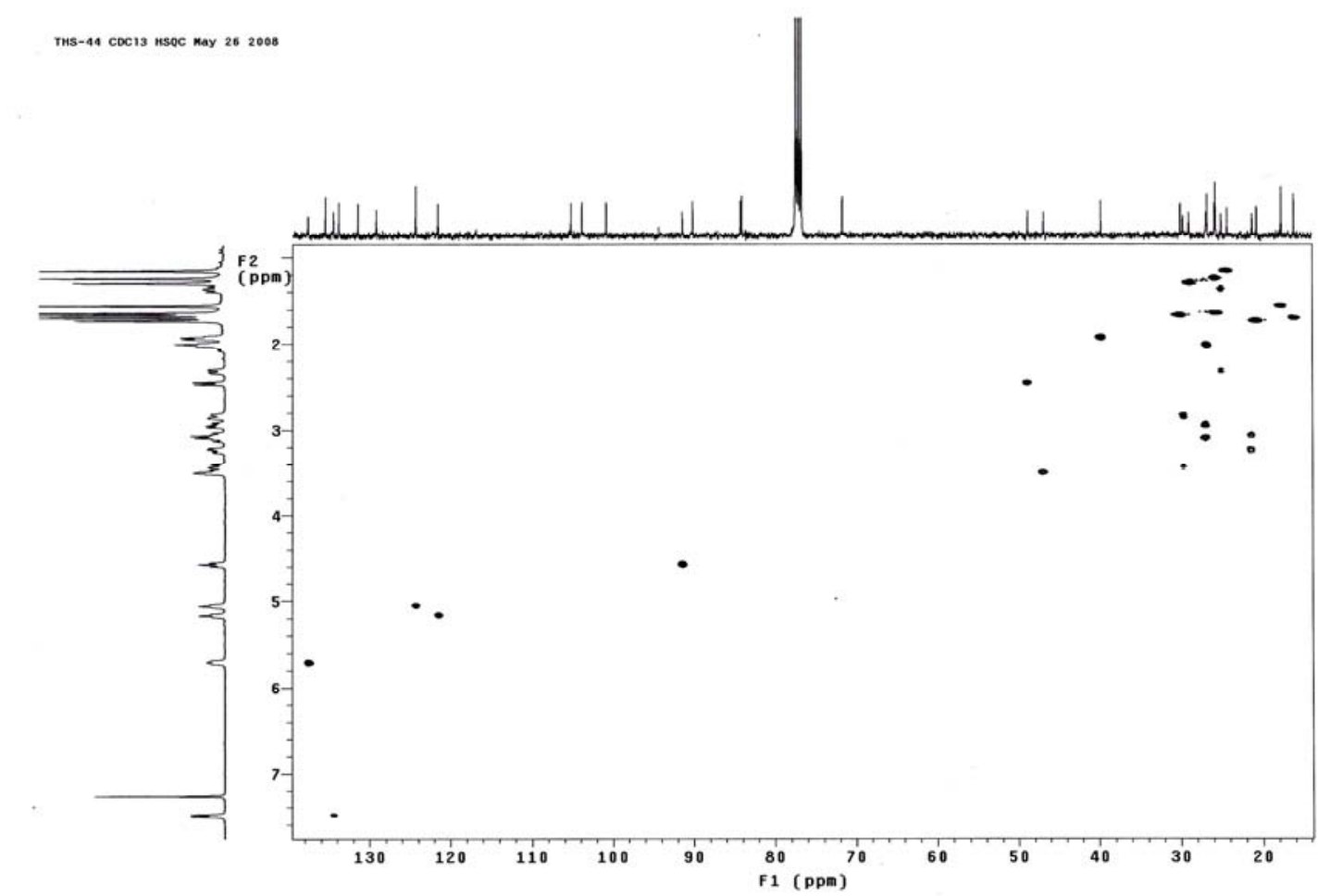


Figure S53. HMBC spectrum (100 MHz, $\left.\mathrm{CDCl}_{3}\right)$ of gambogenific acid (11)

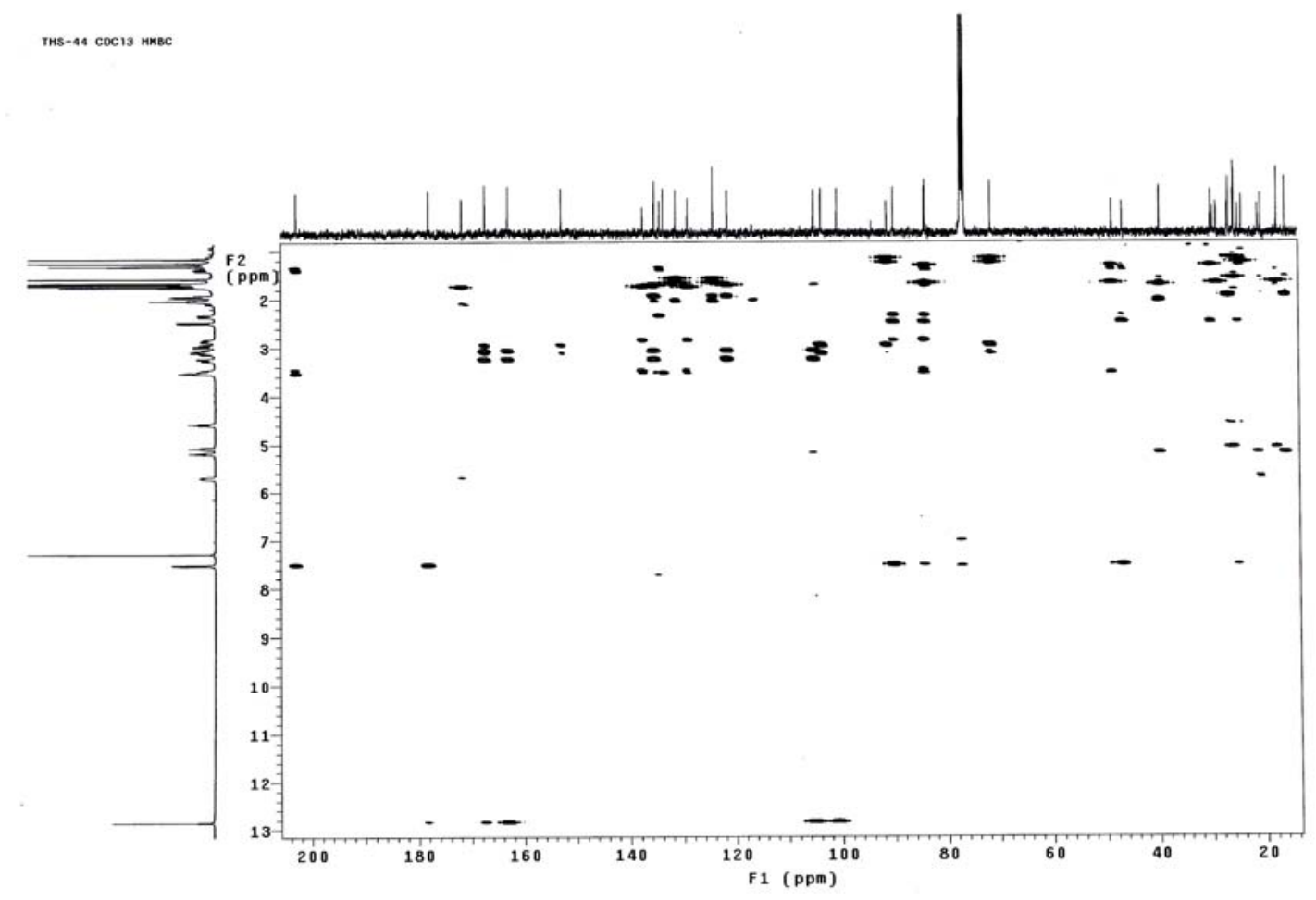

Figure S54. ${ }^{1} \mathrm{H}$ NMR spectrum (300 MHz, $\mathrm{CDCl}_{3}$ ) of 7-methoxyisomorellinol (12)

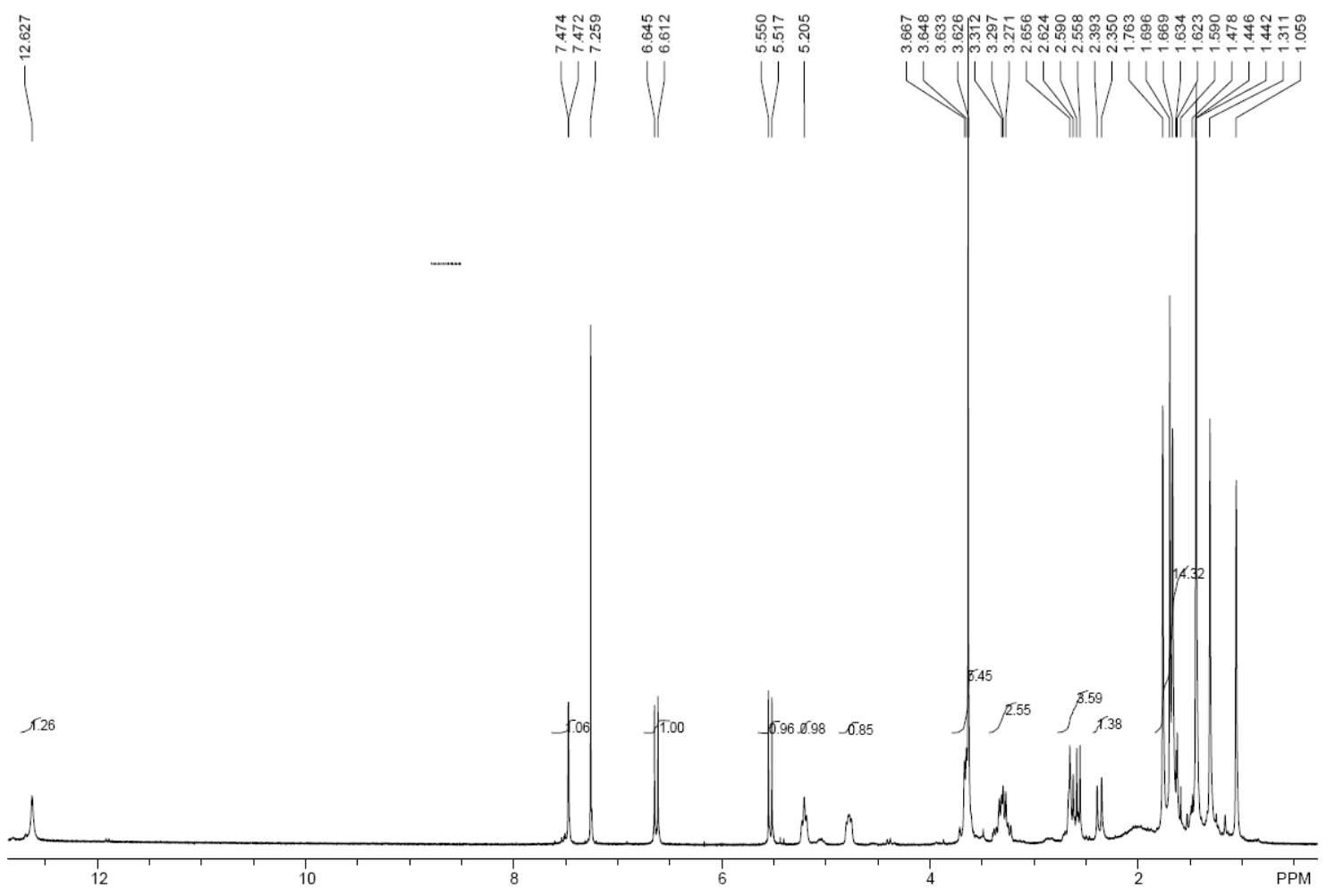


Figure S55. ${ }^{13} \mathrm{C}$ NMR spectrum $\left(100 \mathrm{MHz}, \mathrm{CDCl}_{3}\right)$ of 7-methoxyisomorellinol (12)

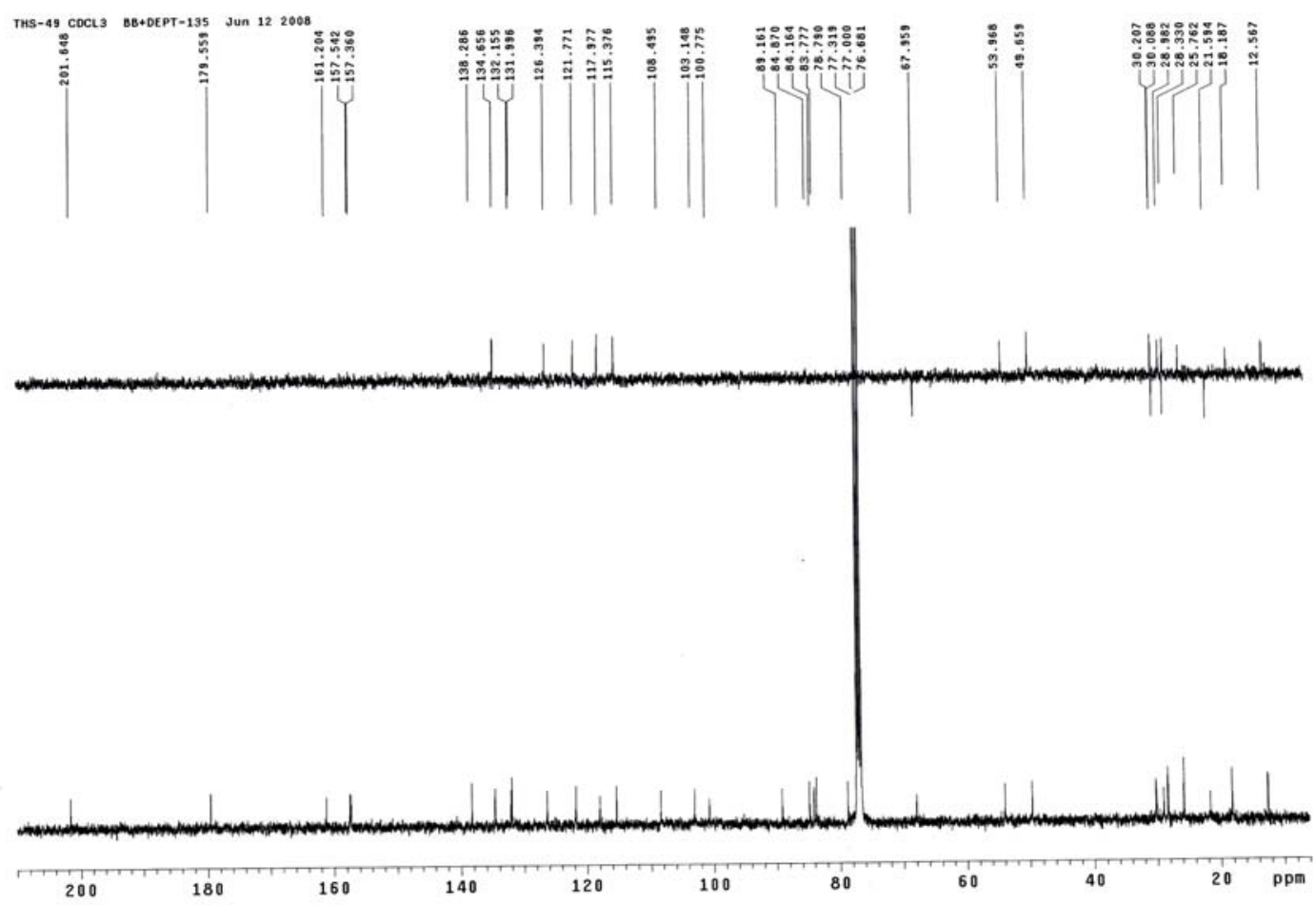

Figure S56. HSQC spectrum (100 MHz, $\left.\mathrm{CDCl}_{3}\right)$ of 7-methoxyisomorellinol (12)

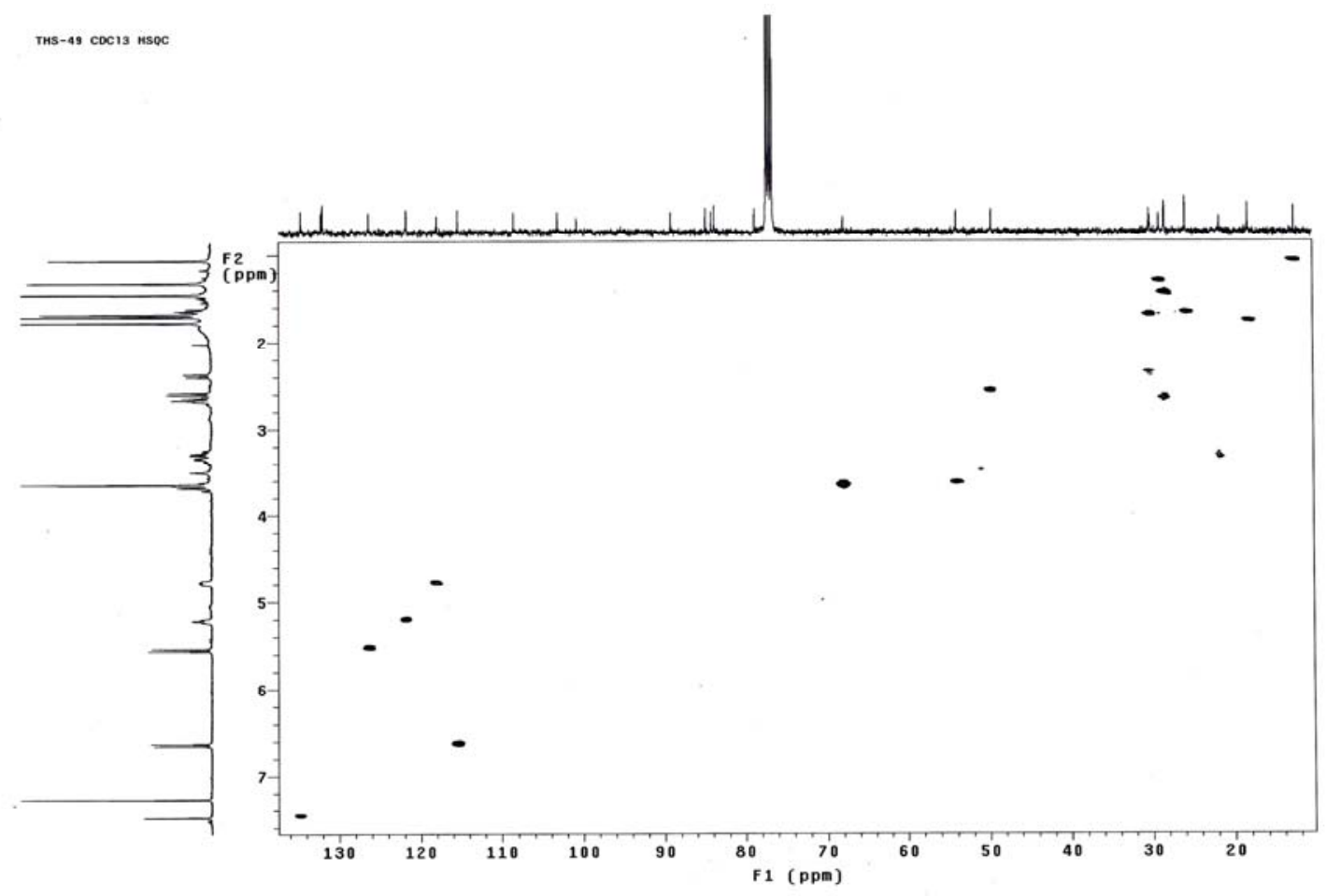


Figure S57. ${ }^{1} \mathrm{H}$ NMR spectrum (400 MHz, $\mathrm{CDCl}_{3}$ ) of 8,8a-dihydro-8-hydroxygambogic acid (13)

$$
\stackrel{+}{\stackrel{0}{亠}}
$$

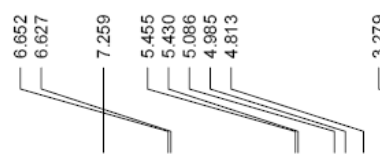

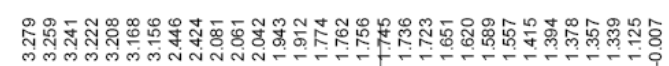

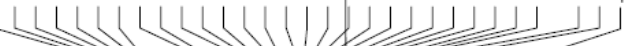

THS 22-1 CDCl3 08.02.28

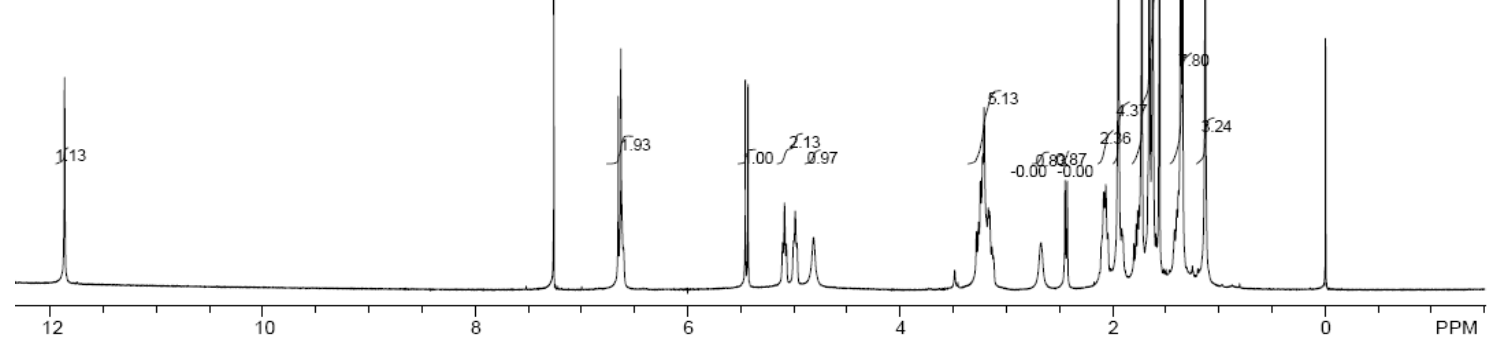

Figure S58. ${ }^{13} \mathrm{C}$ NMR spectrum (100 MHz, $\left.\mathrm{CDCl}_{3}\right)$ of 8,8a-dihydro-8-hydroxygambogic acid (13)

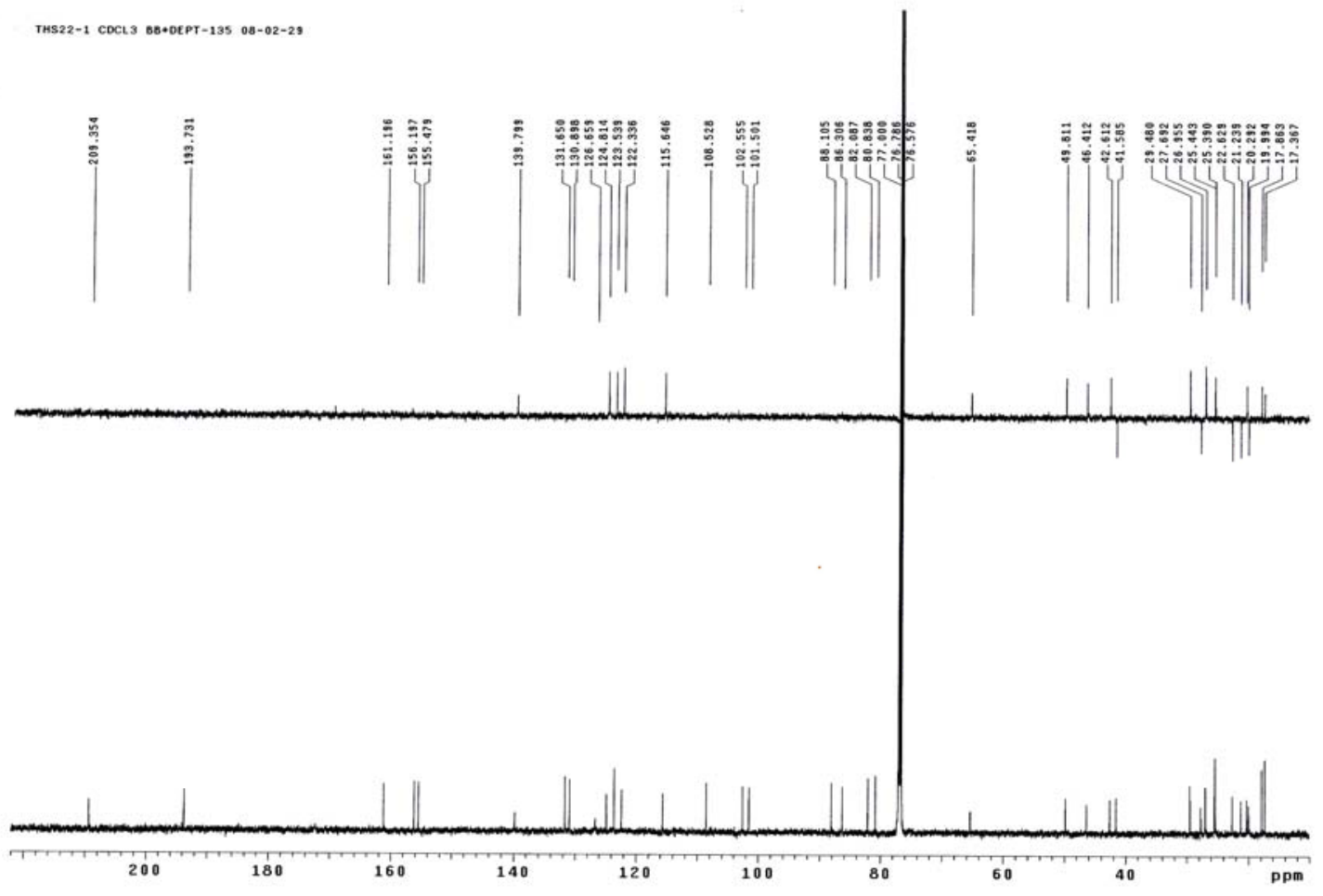


Figure S59. ${ }^{1} \mathrm{H}$ NMR spectrum (400 $\mathrm{MHz}, \mathrm{CDCl}_{3}$ ) of 8,8a-dihydro-8-hydroxygambogic acid isomer (14)

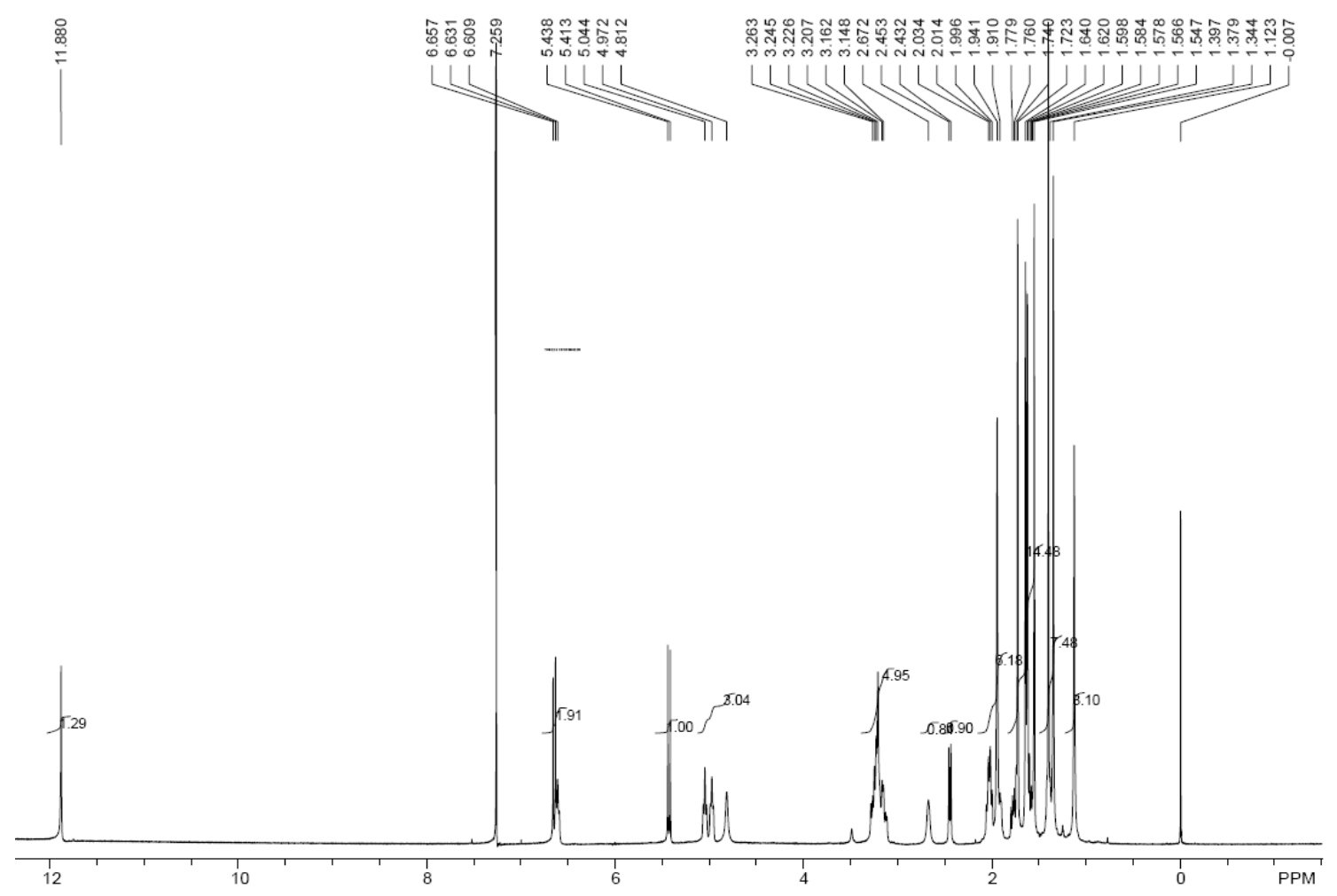

Figure S60. ${ }^{13} \mathrm{C}$ NMR spectrum $\left(100 \mathrm{MHz}, \mathrm{CDCl}_{3}\right)$ of 8,8a-dihydro-8-hydroxygambogic acid isomer (14) 


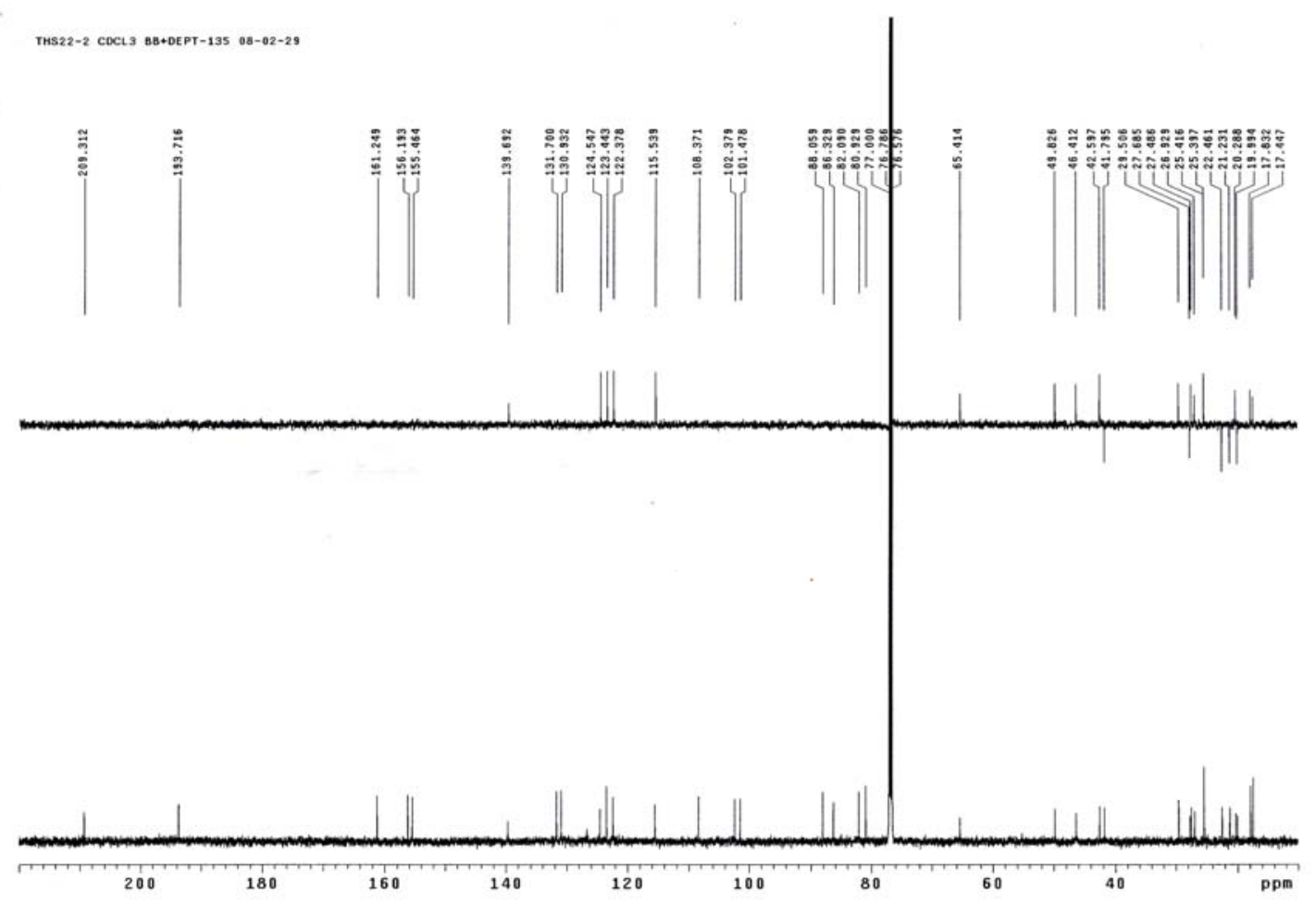

